\title{
GENERALIZED OPERADS AND THEIR INNER COHOMOMORPHISMS ${ }^{1}$
}

\author{
Dennis V. Borisov, Yuri I. Manin \\ Northwestern University, Evanston, USA \\ Max-Planck-Institut für Mathematik, Bonn, Germany,
}

\begin{abstract}
In this paper we introduce a notion of generalized operad containing as special cases various kinds of operad-like objects: ordinary, cyclic, modular, properads etc. We then construct inner cohomomorphism objects in their categories (and categories of algebras over them). We argue that they provide an approach to symmetry and moduli objects in non-commutative geometries based upon these "ring-like" structures. We give a unified axiomatic treatment of generalized operads as functors on categories of abstract labeled graphs. Finally, we extend inner cohomomorphism constructions to more general categorical contexts. This version differs from the previous ones by several local changes (including the title) and two extra references.
\end{abstract}

\section{$\S 0$. Introduction}

0.1. Inner cohomomorphisms of associative algebras. Let $k$ be a field. Consider pairs $\mathcal{A}=\left(A, A_{1}\right)$ consisting of an associative $k$-algebra $A$ and a finite dimensional subspace $A_{1}$ generating $A$. For two such pairs $\mathcal{A}=\left(A, A_{1}\right)$ and $\mathcal{B}=$ ( $\left.B, B_{1}\right)$, define the category $\mathcal{A} \Rightarrow \mathcal{B}$ by the following data.

An object of $\mathcal{A} \Rightarrow \mathcal{B}$ is a pair $(F, u)$ where $F$ is a $k$-algebra and $u: A \rightarrow F \otimes B$ is a homomorphism of algebras such that $u\left(A_{1}\right) \subset F \otimes B_{1}$ (all tensor products being taken over $k$ ).

A morphism $(F, u) \rightarrow\left(F^{\prime}, u^{\prime}\right)$ in $\mathcal{A} \Rightarrow \mathcal{B}$ is a homomorphism of algebras $v: F \rightarrow$ $F^{\prime}$ such that $u^{\prime}=\left(v \otimes \operatorname{id}_{B}\right) \circ u$.

The following result was proved in [Ma3] (see. Prop. 2.3 in Chapter 4):

0.1.1. Theorem. The category $\mathcal{A} \Rightarrow \mathcal{B}$ has an initial object

$$
(E, \delta: A \rightarrow E \otimes B)
$$

\footnotetext{
${ }_{12000}$ Mathematics Subject Classification: 18D50, 18D10, 20C30. Keywords and phrases: Operads, algebras, inner cohomomorphisms, symmetry and deformations in noncommutative geometry.
} 
defined uniquely up to unique isomorphism, together with a finite dimensional subspace $E_{1} \subset E$ generating $E$ and satisfying $\delta\left(A_{1}\right) \subset E_{1} \otimes B_{1}$.

This result can be reinterpreted as follows. Consider another category $\mathrm{PAlg}$ whose objects are finitely generated $k$-algebras together with a presentation $P$, i. e. a surjection $\varphi_{A}: T\left(A_{1}\right) \rightarrow A$ where $A_{1}$ is a finite dimensional linear space, $T\left(A_{1}\right)$ is its tensor algebra, and such that $\operatorname{Ker} \varphi_{A} \cap A_{1}=\{0\}$ so that $A_{1}$ can be considered as a subspace of $A$ (this condition is not really necessary and can be omitted as is done in Sec. 2.) Morphisms $\left(A, \varphi_{A}\right) \rightarrow\left(B, \varphi_{B}\right)$ are algebra homomorphisms $u: A \rightarrow B$ such that $u\left(A_{1}\right) \subset B_{1}$.

This category has a monoidal symmetric structure given by $\left(A, A_{1}\right) \bigcirc\left(B, B_{1}\right):=$ $\left(C, C_{1}\right)$ where $C_{1}=A_{1} \otimes B_{1}$ and $C$ is the subalgebra of $A \otimes B$ generated by $C_{1}$.

Theorem 0.1.1 establishes a functorial bijection between Hom's in this category

$$
\operatorname{Hom}\left(\left(A, A_{1}\right),\left(F, F_{1}\right) \bigcirc\left(B, B_{1}\right)\right) \cong \operatorname{Hom}\left(\left(E, E_{1}\right),\left(F, F_{1}\right)\right)
$$

When there is no risk of confusion, we will omit $A_{1}, B_{1}$ etc in notation, and denote $\left(E, E_{1}\right)$ by $\underline{c o h o m}(A, B)$ so that we have the standard functorial isomorphism in $(P A l g, \bigcirc)$ :

$$
\operatorname{Hom}(A, F \bigcirc B) \cong \operatorname{Hom}(\underline{\operatorname{cohom}}(A, B), F)
$$

defining inner cohomomorphism objects.

The usual reasoning produces functorial comultiplication maps between these objects

$$
\Delta_{A, B, C}: \underline{\operatorname{cohom}}(A, C) \rightarrow \underline{\operatorname{cohom}}(A, B) \bigcirc \underline{\text { cohom }}(B, C),
$$

which are coassociative (compatible with the ordinary associativity constraints for $\bigcirc)$. bra.

In particular $\underline{\text { coend }}(A):=\underline{\operatorname{cohom}}(A, A)$ has the canonical structure of a bialge-

0.2. Interpretation and motivation. Theorem 0.1 .1 was the base of the approach to quantum groups as symmetry objects in noncommutative geometry discussed in [Ma1]-[Ma4]. Namely, consider PAlg as a category of function algebras on "quantum linear spaces" so that the category of quantum linear spaces themselves will be $P A l g^{o p}$. Then cohomomorphism algebras correspond to "matrix quantum spaces", and coendomorphism algeras, after passing to Hopf envelopes, become Hopf algebras of symmetries. (In fact, to obtain the conventional quantum 
groups, one has to add some "missing relations", cf. [Ma2], which also can be done functorially).

In this paper we present several layers of generalizations of Theorem 0.1.1. The first step consists in extending it to operads with presentation and algebras over them, with an appropriate monoidal structure. We are motivated by the same desire to understand symmetry objects ("quantum semi-groups") in non-commutative geometry based upon operads, or algebras over an operad different from $A S S$. In fact, as a bonus we also get an unconventional approach to the deformation theory of operadic algebras.

Namely, let $\mathcal{P}$ be an operad, $V$ a linear space, and $O p E n d V$ the operad of endomorphisms of $V$. The set of structures of a $\mathcal{P}$-algebra upon $V$ is then

$$
\operatorname{Hom}_{\text {Oper }}(\mathcal{P}, O p E n d V) \text {. }
$$

We suggest to consider the object (defined after a choice of spaces of linear generators of both operads)

$$
\underline{\operatorname{cohom}}(\mathcal{P}, \text { OpEnd } V)
$$

as (an operad of functions upon) a noncommutative space. Morphisms of (0.3) to the unit object of the monoidal category of operads will then constitute its set of "classical points" (0.2).

0.3. The phantom of the operad. The next extension of Theorem 0.1.1 involves replacing operads by any of the related structures a representative list of which the reader can find, for example, in [Mar]: May and Markl operads, cyclic operads, modular operads, PROPS, properads, dioperads etc. In this paper, we use for all of them the generic name "generalized operad", or simply "operad", and call operads like May's and Markl's ones "ordinary operads", or "classical operads".

It was long recognized that one variable part of the definition of all these structures is the combinatorics and decoration of underlying graphs ("pasting schemes" of [Mar]), whereas another is the category in which components of the respective operad are supposed to lie. Operad itself for us is a functor from a category of labeled graphs to another symmetric monoidal category, as was stressed already in [KoMa], [GeKa2] and many other works. We decided to spell out the underlying formalism in the Sec. 1 of this paper. If we appear to be too fussy e.g. in the Definition 1.3, this is because we found out that uncritical reliance on illustrative pictures can be really misleading.

One can and must approach operadic constructions from various directions and with various stocks of analogies. In this paper, we look at operads, especially those 
with values in abelian categories, as analogs of associative rings; collections are analogs of their generating spaces. We imagine various noncommutative geometries based upon operads, and are interested in naturally emerging symmetry and moduli objects in these noncommutative geometries.

But of course there are many more different intuitive ideas related to operads.

a) Operads provide tools for studying general algebraic structures determined by a basic set, a family of composition laws, and a family of constraints imposed upon these laws.

b) Operads embody a categorification of graph theory which can be used to study knot invariants, Feynman perturbation series etc.

c) Operads and their algebras are a formalization of computational processes and devices, in particular, tensor networks and quantum curcuits, cf. [MarkSh], [Zo] and references therein. With this in mind, we describe general endomorphism operads in 2.5 below.

It is interesting to notice that the classical theory of recursive functions must refer to a very special and in a sense universal algebra over a non-linear "computational operad", but nobody so far was able to formalize the latter. Main obstacle is this: a standard description of any partially recursive function produces a circuit that may contain cycles of an a priori unknown multiplicity and eventually infinite subprocesses producing no output at all.

0.4. Plan of the paper. In Sec. 1, we discuss the background topics. The centerpieces of the first part related to graphs are definitions 1.2.1 and 1.3, and the rest is devoted to collections and operads.

In Sec. 2, we state and prove our main theorems in two contexts: for operads in abelian categories and for algebras. However, the latter requires serious additional restrictions. We also discuss in 2.7, 2.8 some explicit descriptions of cohomomorphism objects, whenever they are known, in particular, for quadratic and more general $N$-homogeneous algebras and operads.

Notice that Theorem 0.1.1 was extended in [GrM] to include the case of twisted tensor products of algebras: see also [Ma4] where the latter appeared in the construction of the De Rham complex of quantum groups and spaces. For further developments see $[\mathrm{GrM}]$ and references therein. Similar generalizations might exist for operads as well.

Sec. 3 is dedicated to the next layer of generalizations. Namely, operads considered in Sec. 2 and Sec. 3 can be viewed as algebras over a triple whose main 
component is the endofunctor $\mathcal{F}$ on a category of collections, described in 1.5.5 and 1.5.6. Categories of collections in this context are abelian and endowed with a symmetric monoidal structure. In Sec. 3, we consider more general triples and, in particular, do not assume that the category on which the relevant endofuctor acts, is abelian.

This line of thought is continued in Sec. 4, where in particular operads with values in various categories of algebras are considered. This is partly motivated by the quantum cohomology operad.

Finally, in Appendix we briefly treat Markl's list ([Mar], p. 45) in terms of labeled graphs and functors on them.

Acknowledgements. Yu. M. gratefully acknowledges Bruno Vallette's comments on the preliminary drafts of this paper and discussions with him, in particular related to [Va2]. His numerous suggestions are incorporated in the text. D. B. would like to thank Ezra Getzler for his remarks relating to model categories and the theory of operads in 2-categorical setting.

\section{$\S 1$. Background}

1.1. Graphs. We define objects of the category of (finite) graphs as in [KoMa], [BeMa], [GeKa2]. Geometric realizations of our graphs are not necessarily connected. This allows us to introduce a monoidal structure "disjoint union" on graphs (cf. 1.2.4), and to consider certain morphisms such as graftings and mergers which were not needed in [GeKa2] but arise naturally in more general types of operads. Moreover, our notion of a graph morphism is strictly finer than that considered in the literature: as a part of a morphism, we consider the involution $j_{h}$ in the Definition 1.2.1 below. Our basic category of sets is assumed to be small.

1.1.1. Definition. A graph $\tau$ is a family of finite sets and maps $\left(F_{\tau}, V_{\tau}, \partial_{\tau}, j_{\tau}\right)$ Elements of $F_{\tau}$ are called flags of $\tau$, elements of $V_{\tau}$ are called vertices of $\tau$. The map $\partial_{\tau}: F_{\tau} \rightarrow V_{\tau}$ associates to each flag a vertex, its boundary. The map $j_{\tau}: F_{\tau} \rightarrow F_{\tau}$ is an involution: $j_{\tau}^{2}=i d$.

Marginal cases. If $V_{\tau}$ is empty, $F_{\tau}$ must be empty as well. This defines an empty graph. To the contrary, $F_{\tau}$ might be empty whereas $V_{\tau}$ is not. In [GeKa2] and other places, in order to treat various units, a "non-graph" with one flag and no vertices is considered. Its role in our constructions sometimes can be played by the empty graph.

Edges, tails, corollas. One vertex graphs with identical $j_{\tau}$ are called corollas. Let $v$ be a vertex of $\tau, F_{\tau}(v):=\partial_{\tau}^{-1}(v)$. Then $\tau_{v}:=\left(F_{\tau}(v),\{v\}\right.$, evident $\partial$, identical $\left.j\right)$ 
is a corolla, which is called the corolla of $v$ in $\tau$.

Flags fixed by $j_{\tau}$ form the set of tails of $\tau$ denoted $T_{\tau}$.

Two-element orbits of $j_{\tau}$ form the set $E_{\tau}$ of edges of $\tau$. Elements of such an orbit are called halves of the respective edge.

1.1.2. Geometric realization of a graph. First, let $\tau$ be a corolla. If its set of flags is empty, its geometric realization $|\tau|$ is, by definition, a point. Otherwise construct a disjoint union of segments $\left[0, \frac{1}{2}\right]$ and identify in it all points 0 . This is $|\tau|$. The image of 0 thus becomes the geometric realization of the unique vertex of $\tau$.

Generally, to construct $|\tau|$ take a disjoint union of geometric realizations of corollas of all vertices and identify points $\frac{1}{2}$ of any two flags forming an orbit of $j_{\tau}$.

A graph $\tau$ is called connected (resp. simply connected, resp. tree etc) iff its geometric realization is such. In the same vein, we can speak about connected components of a graph etc. Vertices $v$ with empty $F_{\tau}(v)$ are considered as connected components.

1.2. Morphisms of graphs and monoidal category $G r$. Let $\tau, \sigma$ be two graphs.

1.2.1. Definition. A morphism $h: \tau \rightarrow \sigma$ is a triple $\left(h^{F}, h_{V}, j_{h}\right)$, where $h^{F}: F_{\sigma} \rightarrow F_{\tau}$ is a contravariant map, $h_{V}: V_{\tau} \rightarrow V_{\sigma}$ is a covariant map, and $j_{h}$ is an involution on the set of tails of $\tau$ contained in $F_{\tau} \backslash h^{F}\left(F_{\sigma}\right)$. This data must satisfy the following conditions.

(i) $h^{F}$ is injective, $h_{V}$ is surjective.

(ii) The image $h^{F}\left(F_{\sigma}\right)$ and its complement $F_{\tau} \backslash h^{F}\left(F_{\sigma}\right)$ are $j_{\tau}$-invariant subsets of flags. The involution $j_{h}$ fixes no tail in $F_{\tau} \backslash h^{F}\left(F_{\sigma}\right)$.

It will be convenient to extend $j_{h}$ to other flags in $F_{\tau}$ by identity.

We will say that $h$ contracts all flags in $F_{\tau} \backslash h^{F}\left(F_{\sigma}\right)$. If two flags in $F_{\tau} \backslash h^{F}\left(F_{\sigma}\right)$ form an edge, we say that this edge is contracted by h. If two tails in $F_{\tau} \backslash h^{F}\left(F_{\sigma}\right)$ form an orbit of $j_{h}$, we say that it is a virtual edge contracted by $h$.

(iii) If a flag $f_{\tau}$ is not contracted by $h$, that is, has the form $h^{F}\left(f_{\sigma}\right)$, then $h_{V}$ sends $\partial_{\tau} f_{\tau}$ to $\partial_{\sigma} f_{\sigma}$. Two vertices of a contracted edge (actual or virtual) must have the same $h_{V}$-image.

(iv) The bijection $h_{F}^{-1}: h^{F}\left(F_{\sigma}\right) \rightarrow F_{\sigma}$ maps edges of $\tau$ to edges of $\sigma$.

If it maps a pair of tails of $\tau$ to an edge of $\sigma$, we will say that $h$ grafts these tails. 
The composition of two morphisms corresponds to the set-theoretic composition of the respective maps $h^{F}$ and $h_{V}$, and taking the union of two sets of virtual edges.

The resulting category is denoted $G r$.

1.2.2. Geometric realization of a morphism. On geometric realizations, the action of $h$ can be visualized as follows: we construct a subgraph of $\tau$ consisting only of flags in $h^{F}\left(F_{\sigma}\right)$, then produce its quotient, and then identify this subquotient with $\sigma$ using $\left(h^{F}\right)^{-1}$.

The shortest description of this subquotient is this: merge in $|\tau|$ all vertices belonging to each one fiber of $h_{V}$, then delete all flags which are contracted by $h$.

It is easy to see that $\left(h^{F}\right)^{-1}$ identifies the geometric graph thus obtained with $|\sigma|$.

This short and intuitive description may be misleading for important concrete categories $\Gamma$ of labeled graphs (see Definition 1.3). It might happen that such a category does not allow morphisms which simply delete flags, and/or does not allow morphisms that merge two vertices without contracting a path of edges (actual or virtual) connecting such a pair.

The following sequence of steps has more chances to represent a sequence of morphisms in $\Gamma$.

a) In each $j_{h}$-orbit, graft tails of $|\tau|$ belonging to $F_{\tau} \backslash h^{F}\left(F_{\sigma}\right)$ making an actual edge from the virtual one.

Contract to a point each connected component of the union of all actual or virtual edges whose halves belong to $F_{\tau} \backslash h^{F}\left(F_{\sigma}\right)$. This point becomes a new vertex.

Besides contracted halves of edges, all other flags adjacent to various vertices of the contracted component are retained and become adjacent to the vertex that is the image of this component. Thus the set of remaining flags consists exactly of the (geometric realizations of) $h^{F}\left(F_{\sigma}\right)$.

From Def. 1.2.1 (iii) it follows that all flags adjacent to the new vertex are sent by $\left(h^{F}\right)^{-1}$ to a subset of flags adjacent to one and the same vertex of $\sigma$.

b) Graft loose ends of each pair of remaining tails that are grafted by $h$.

c) Merge together those vertices of the obtained (geometric) graph whose preimages are sent by $h_{V}$ to one and the same vertex of $\tau$.

Finally, identify the resulting subquotient of $|\tau|$ with $|\sigma|$ in such a way that on flags this map becomes $\left(h^{F}\right)^{-1}: h^{F}\left(F_{\sigma}\right) \rightarrow F_{\tau}$.

Notice that all steps a) - c) could have been done in arbitrary order, for example c), b), a), with the same result. Notice also that in the geometric realization, 
no trace of $j_{h}$ is remained: the virtual edges vanished, but we do not know how their halves were paired. This information is encoded only in the combinatorial description involving $j_{h}$.

Each step above is in fact a geometric realization of a morphism in $G r$. We will now describe formally the respective classes of morphisms.

1.2.3. Contractions, graftings, mergers. a) Virtual contractions. A morphism $h: \tau \rightarrow \sigma$ is called a virtual contraction, if $F_{\tau} \backslash h^{F}\left(F_{\sigma}\right)$ consists only of tails, restriction of $j_{\tau}$ on $h^{F}\left(F_{\sigma}\right)$ coincides with the image of $j_{\sigma}$, and $h_{V}$ is a bijection.

b) Contractions and full contractions. A morphism is called a contraction, if $h^{F}$ is bijective on tails, and if for any $v \in V_{\sigma}$, any two different vertices in $h_{V}^{-1}(v)$ are connected by a path consisting of edges contracted by $h$.

Let $\sigma$ be a graph. Define $\tau$ as follows: $F_{\tau}:=T_{\sigma}, V_{\tau}:=\{$ connected components of $\sigma$ \}. Let $h^{F}$ be the identical injection $T_{\sigma} \rightarrow F_{\sigma}$. Let $h_{V}$ send any vertex to the connected component in which it is contained. The resulting morphism is called the full contraction. Its image is a union of corollas, tails of $\sigma$ are distributed among them as they are among connected components of $\sigma$. Morphisms isomorphic to such ones are also called full contractions (of their source).

c) Grafting and total grafting. A morphism $h$ is called a grafting, if $h^{F}$ and $h_{V}$ are bijections.

Let $\tau$ be a graph. Denote by $\sigma:=\coprod_{v \in V_{\tau}} \tau_{v}$ the disjoint union of corollas of all its vertices. Formally, $F_{\sigma}=F_{\tau}, V_{\sigma}=V_{\tau}, \partial_{\sigma}=\partial_{\tau}$, and $j_{\sigma}=i d$. Let $h: \sigma \rightarrow \tau$ consist of identical maps. Such a morphism is called total grafting, and we will reserve for it a special notation:

$$
\circ_{\tau}: \coprod_{v \in V_{\tau}} \tau_{v} \rightarrow \tau
$$

It is defined uniquely by its target $\tau$, up to unique isomorphism identical on $\tau$. Any isomorphism of targets induces an isomorphism of such morphisms. Its formal inversion cuts all edges of $\tau$ in half. Morphisms isomorphic to such one are also called total grafting (of their target).

d) Mergers and full mergers. A morphism $h$ is called a merger, if $h^{F}$ is bijective and identifies $j_{\sigma}$ with $j_{\tau}$.

A full merger projects all vertices into one. All edges become loops; tails remain tails. 
Mergers play no role in the theory of ordinary operads, but are essential for treating PROPs, cf. Appendix.

Each graph $\tau$ admits a morphism to a corolla bijective on tails

$$
\operatorname{con}_{\tau}: \tau \rightarrow \operatorname{con}(\tau)
$$

It can be described as the full contraction of $\tau$ followed by the full merger. It is defined uniquely by its source $\tau$, up to unique isomorphism identical on $\tau$. Any isomorphism of sources induces a unique isomorphism of such morphisms.

Isomorphisms constitute the intersection of all four classes.

A combinatorial argument imitating 1.2.2 shows that any morphism can be decomposed into a product of a virtual contraction, a contraction, a grafting and a merger. As soon as the order of the types of morphisms is chosen, one can define such a decomposition in a canonical way, up to a unique isomorphism.

1.2.4. Disjoint union as a monoidal structure on $G r$. Disjoint union of two abstract sets having no common elements is an obvious notion. If we want to extend it to "all" sets, an appropriate formalization is that of a symmetric monoidal structure "direct sum" with empty set as the unit object. It exists, but is neither unique, nor completely obvious: what is the "disjoint union of a set with itself"? One way to introduce such a structure is described in [Bo2], Example 6.1.9.

We will focus on a small category of finite sets of all cardinalities and sketch the following method which neatly accounts for proliferation of combinatorics of symmetric groups in the standard treatments of operads.

The small category of finite sets of all cardinalities consisting of

$$
\emptyset,\{1\},\{1,2\}, \ldots,\{1,2, \ldots, n\}, \ldots
$$

admits a monoidal structure "disjoint union" Џ given by

$$
\{1,2, \ldots, m\} \coprod\{1,2, \ldots, n\}:=\{1,2, \ldots, m+n\}
$$

and evident commutativity and associativity constraints. For example, identification of $X \coprod Y$ with $Y \coprod X$ proceeds by putting all elements of $Y$ before those of $X$ and retaining the order inside groups. Empty set is the unit of this monoidal structure.

In these terms, it is clear how to extend this construction to the category of "all" totally ordered finite sets, and then to drop the orderings by passing to appropriate 
colimits. Thus, we can endow the category of finite sets by a monoidal structure which we keep denoting Ц and calling "disjoint union".

This monoidal structure can then be extended to $G r: \sigma \coprod \tau$ is determined by disjoint unions of their respective flag and vertex sets, and $\partial, j$ act on both parts as they used to.

Finally, for any finite family $\left\{\tau_{s} \mid s \in S\right\}$, we can define $\coprod_{s} \tau_{s}$ functorially in $\left\{\tau_{s}\right\}$ and $S$ as is spelled out in [DeMi].

1.2.5. Atomization of a morphism. Let $h: \tau \rightarrow \sigma$ be a morphism of graphs. We define its atomization as a commutative diagram of the following form:

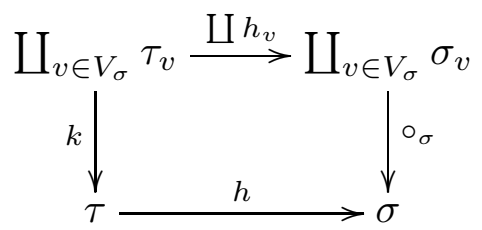

Here $\sigma_{v}$ is the corolla of a vertex $v \in V_{\sigma}, \circ_{\sigma}$ is the total grafting morphism, and the remaining data are constructed as follows.

Graph $\tau_{v}$. We put for $v \in V_{\sigma}$ :

$$
\begin{gathered}
F_{\tau_{v}}:=\left\{f \in F_{\tau} \mid h_{V}\left(\partial_{\tau} f\right)=v\right\}, V_{\tau_{v}}:=\left\{w \in V_{\tau} \mid h_{V}(w)=v\right\} \\
\partial_{\tau_{v}}=\left.\partial_{\tau}\right|_{\tau_{v}}, j_{\tau_{v}}=\left.j_{\tau}\right|_{\tau_{v}} .
\end{gathered}
$$

Morphism $h_{v}: \tau_{v} \rightarrow \sigma_{v}$. We put

$$
h_{v}^{F}:=\left.h^{F}\right|_{F_{\sigma_{v}}}: F_{\sigma_{v}} \rightarrow F_{\tau_{v}}, h_{v, V}:=\left.h_{V}\right|_{V_{\tau_{v}}}: V_{\tau_{v}} \rightarrow V_{\sigma_{v}}, j_{h_{v}}:=\left.j_{h}\right|_{F_{\tau_{v}}} .
$$

Morphism $k$. By definition, $k^{F}$ and $k_{V}$ are identical maps, hence $k$ is a grafting.

1.2.6. Heredity. Let now $\circ_{\sigma}: \coprod_{v \in V_{\sigma}} \sigma_{v} \rightarrow \sigma$ be a total grafting morphism. Assume that we are given a family of morphisms $h_{v}: \tau_{v} \rightarrow \sigma_{v}, v \in V_{\sigma}$. Then this data can be uniquely extended to the atomization diagram (1.3) of a morphism $h: \sigma \rightarrow \tau$.

1.3. Definition. An abstract category of labelled graphs is a category $\Gamma$ endowed with a functor $\psi: \Gamma \rightarrow G r$ satisfying the following conditions:

(i) $\Gamma$ is endowed with a monoidal structure which $\psi$ maps to the disjoint union in $\mathrm{Gr}$. It will be denoted by the same sign $\amalg$. 
(ii) $\psi$ is faithful: if two morphisms with common source and target become equal after applying $\psi$, they are equal.

(iii) Call a $\Gamma$-corolla any object $\tau$ of $\Gamma$ such that $\psi(\tau)$ is a corolla. Any object $\tau \in \Gamma$ admits a morphism to a $\Gamma$-corolla

$$
\operatorname{con}_{\tau}: \tau \rightarrow \operatorname{con}(\tau)
$$

which is a lift to $\Gamma$ of the diagram of the form (1.2) with the source $\psi(\tau)$. It is defined uniquely up to unique isomorphism identical on $\tau$.

(iv) Any object of $\Gamma$ is the target of a morphism from a disjoint union of $\Gamma-$ corollas

$$
\circ_{\tau}: \coprod_{v \in V_{\psi(\tau)}} \tau_{v} \rightarrow \tau
$$

which is a lift to $\Gamma$ of the diagram of the form (1.1) with the target $\psi(\tau)$. It is defined uniquely up to unique isomorphism identical on $\tau$.

(v) Any morphism $h: \tau \rightarrow \sigma$ can be embedded into a commutative diagram of the form (1.3) which is a lift of the atomization diagram for the morphism $\psi(h)$ : $\psi(\tau) \rightarrow \psi(\sigma)$. Such a diagram is defined uniquely up to unique isomorphism.

(vi) Moreover, assume that we are given $\sigma$ in $\Gamma$, and for each $\Gamma$-corolla $\sigma_{v}$, $v \in V_{\psi(\sigma)}$, a morphism $h_{v}: \tau_{v} \rightarrow \sigma_{v}$ where $\left\{\tau_{v}\right\}$ is a family of objects of $\Gamma$. Then there exists a morphism $\tau \rightarrow \sigma$ in $\Gamma$ such that all this data fit into (a lift of) the atomization diagram (1.3). Moreover, $\tau$ and $\sigma$ are defined uniquely up to unique isomorphism.

The last requirement formalizes what Markl calls "hereditary" property in [Mar], p. 45.

1.3.1. Comments. a) It is helpful (and usually realistic) to imagine any object $\sigma \in \Gamma$ as a pair consisting of the "underlying graph" $\psi(\sigma)$ and an additional structure on the components of $\psi(\sigma)$ such as decorating vertices by integers, a cyclic order on flags adjoining to a vertex, etc (see examples below). We will generally refer to such a structure as "labeling".

As a rule, existence of labeling of a given type and/or additional algebraic properties required for a treatment a certain type of operadic structure put some restrictions upon underlying graphs so that $\psi$ need not be surjective on objects. On the other hand, if these restrictions are satisfied, there might be many different compatible labeling on the same underlying graph so that $\psi$ need not be injective on objects either. 
The functor $\psi$ on objects simply forgets labeling.

b) In the same vein, any morphism $\sigma \rightarrow \tau$ in $\Gamma$ should be imagined as a morphism $\psi(\sigma) \rightarrow \psi(\tau)$ of underlying graphs satisfying some constraints of two types: purely geometric ones which can be stated in $G r$, and certain compatibility conditions with labelings (see below). This is the content of condition (ii) above.

c) Let $\sigma \in \Gamma$. Slightly abusing the language, we will call flags, vertices, edges etc. of $\psi(\sigma)$ the respective components of $\sigma$, and write, say, $V_{\sigma}$ in place of $V_{\psi(\sigma)}$. Similar conventions will apply to morphisms in $\Gamma$. By extension, $\sigma$ is called a $\Gamma$-corolla (resp. tree etc), if $\psi(\sigma)$ is a corolla (resp. tree etc).

1.3.2. Examples of labeling. a) Oriented graphs. Any map $F_{\sigma} \rightarrow\{$ in ,out $\}$ such that halves of any edge are oriented by different labels, is called an orientation of $\sigma$. On the geometric realization, a flag marked by in (resp. out) is oriented towards (resp. outwards) its vertex.

Tails of $\sigma$ oriented in (resp. out) are called inputs (resp. outputs) of $\sigma$. Similarly, $F_{\sigma}(v)$ is partitioned into inputs and outputs of the vertex $v$.

Consider an orientation of $\sigma$. Its edge is called an oriented loop, if both its halves belong to the same vertex. Otherwise an oriented edge starts at a source vertex and ends at a different target vertex.

More generally, a sequence of pairwise distinct edges $e_{1}, \ldots, e_{n}$, is called a simple path of length $n$, if $e_{i}$ and $e_{i+1}$ have a common vertex, and the $n-1$ vertices obtained in this way are pairwise distinct. If moreover $e_{1}$ and $e_{n}$ also have a common vertex distinct from the mentioned ones, this path is a wheel of length $n$. A loop is a wheel of length one. Edges in a wheel are endowed only with a cyclic order up to inversion.

Clearly, all edges in a path (resp. a wheel) can be oriented so that the source of $e_{i+1}$ is the target of $e_{i}$.

If the graph is already oriented, the induced orientation on any path (resp. wheel) either has this property or not. Respectively, the wheel is called oriented or not.

A morphism of oriented graphs $h$ is a morphism of graphs such that $h^{F}$ is compatible with orientations.

b) Directed graphs. An oriented graph $\sigma$ is called directed if it satisfies the following condition:

On each connected component of the geometric realization, one can define a continuous real valued function ("height") in such a way that moving in the direction of orientation along each flag decreases the value of this function. 
In particular, a directed graph has no oriented wheels.

Notice that, somewhat counterintuitively, a directed graph is not necessarily oriented "from its inputs to its outputs" as is usually shown on illustrating pictures. In effect, take a corolla with only in flags and another corolla with only out flags, and graft one input to one output. The resulting graph is directed (check this) although its only edge is oriented from global outputs to global inputs.

This is one reason why it is sometimes sensible to include in a category $\Gamma$ of directed graphs only those, which have at least one input and least one output at each vertex (cf. the definition of reduced bimodules in Sec. 1.1 ov [Va1]).

Another reason for excluding certain marginal ("unstable") types of labeled corollas might be our desire to ensure essential finiteness of the categories denoted $\Rightarrow \sigma$ in Sec. 1.5.5 (cf. a description of unstable modular corollas below). In a category of (disjoint unions of) directed trees, for example, this leads to the additional requirement: corolla of any vertex has at least three flags.

This requirement might lead to some technical problems if we want to consider unital versions of our operads.

c) Genus labeling. A genus labeling of $\sigma$ is a map $g: V_{\sigma} \rightarrow \mathbf{Z}_{\geq 0}, v \mapsto g_{v}$. The genus of a connected labeled graph $\sigma$ is defined as

$$
g(\sigma):=\sum_{v \in V_{\sigma}} g_{v}+\operatorname{rk} H_{1}(|\sigma|)=\sum_{v \in V_{\sigma}}\left(g_{v}-1\right)+\operatorname{card} E_{\sigma}+1 .
$$

Genus labeled graphs (or only connected ones) are called modular graphs. Corolla of any vertex of a modular graph is a modular graph.

A morphism of modular graphs is a morphism of graphs compatible with labeling in the following sense. Contraction of a looping edge raises the genus of its vertex by one. Contraction of a non-looping edge prescribes to the emerging vertex the sum of genera of two ends of the edge. Finally, grafting flags does not change genera of vertices.

Thus, a morphism between two connected modular graphs can exist only if their genera coincide.

A modular corolla with vertex of genus $g$ and $n$ flags is called stable iff $2 g-2+n>$ 0. A modular graph is called stable, iff corollas of all its vertices are stable.

d) Colored graphs. Let $I$ be an abstract set (elements of which are called colors). An $I$-colored graph is a graph $\tau$ together with a map $F_{\tau} \rightarrow I$ such that two halves 
of each edge get the same color. Morphisms are restricted by the condition that $h^{F}$ preserves color.

In [LoMa], a topological operad was studied governed by a category of colored graphs with two-element $I=\{$ black, white $\}$. Halves of an edge in this category are always white.

e) Cyclic labeling. A cyclic labeling of a graph $\tau$ is a choice of cyclic order upon each set $F_{\tau}(v)$. Alternatively, it is a family of bijections $F_{\tau}(v) \rightarrow \mu_{|v|}$ where $\mu_{|v|}$ is the group of roots of unity (in $\mathbf{C}$ ) of degree $|v|:=F_{\tau}(v)$; two maps which differ by a multiplication by a root of unity define the same labeling. Yet another description identifies a cyclic labeling of $\tau$ with a choice of planar structure for each corolla $\tau_{v}$, that is, an isotopy class of embeddings of $\left|\tau_{v}\right|$ into an oriented plane.

In a category of cyclic labeled graphs, mergers are not allowed, whereas contractions, say, of one edge, should be compatible with cyclic labeling in an evident way: say $(0,1, \ldots, m)$ and $(0, \overline{1}, \ldots, \bar{n})$ turn into $(1, \ldots, m, \overline{1}, \ldots, \bar{n})$, where by 0 we denoted the contracted halves of the same edge in two corollas.

An interesting variant of cyclic labeling is unoriented cyclic labeling (it has nothing to do with orientation of the graph itself): the cyclic orders $(0,1, \ldots, m)$ and $(m, m-1, \ldots, 0)$ are considered equivalent. In this version, contraction of an edge leads generally to two different morphisms in $\Gamma$, with two different targets.

Combinatorics of unoriented cyclic labeled trees is very essential in the description of the topological operad of real points $\bar{M}_{0, *}(\mathbf{R})$, cf. [GoMa].

1.4. Ground categories $\mathcal{G}$. Operads of various types in this paper will be defined as certain functors from a category of labeled graphs $\Gamma$ to a symmetric monoidal category $(\mathcal{G}, \otimes)$ which will be called ground category. The simplest example is that of finite-dimensional vector spaces over a field, or that of finite complexes of such spaces.

In order to ensure validity of various constructions we will have to postulate (locally) some additional properties of $(\mathcal{G}, \otimes)$, the most important of which are contained in the following list. At this stage, we do not assume that all of them, or some subset of them, hold simultaneously.

a) Existence of a unit object.

b) Existence of internal cohom objects $\underline{\operatorname{cohom}}(X, Z)$ for any objects $X, Z$ in $\mathcal{G}$. By definition, they fit into functorial isomorphisms

$$
\operatorname{Hom}_{\mathcal{G}}(X, Y \otimes Z)=\operatorname{Hom}_{\mathcal{G}}(\underline{\operatorname{cohom}}(X, Z), Y)
$$


These isomorphisms are established by composition with coevaluation morphisms

$$
c=c_{X, Z}: X \rightarrow \underline{\operatorname{cohom}}(X, Z) \otimes Z
$$

(cf. the diagram (2.3) in Section 2 below).

c) Existence of countable coproducts such that $\otimes$ is distributive with respect to these coproducts.

d) Existence of finite (and sometimes infinite) colimits.

e) $\mathcal{G}$ is an abelian category, $\otimes$ is an additive bifunctor exact in each argument.

f) $\mathcal{G}$ is a closed model category.

1.5. $\Gamma \mathcal{G}$-collections. We will denote by $\Gamma C O R$ the subcategory (groupoid) of $\Gamma$ consisting of $\Gamma$-corollas and isomorphisms between them.

1.5.1. Definition. $A \Gamma \mathcal{G}$-collection $A_{1}$ is a functor $A_{1}: \Gamma C O R \rightarrow \mathcal{G}$. A morphism of $\Gamma \mathcal{G}$-collections $A_{1} \rightarrow B_{1}$ is a functor morphism (natural transformation.)

The category of $\Gamma \mathcal{G}$-collections will be denoted $\Gamma \mathcal{G} C O L L$.

1.5.2. Examples. a) If $\Gamma$ is the category of stable modular graphs, the category $\Gamma \mathcal{G C O L L}$ is equivalent to the category of double sequences $A_{1}((g, n))$ of objects of $\mathcal{G}$ endowed with an action of $\mathbf{S}_{n}$ upon $A_{1}((g, n))$. A morphism $A_{1} \rightarrow B_{1}$ is a sequence of morphisms in $\mathcal{G}, A_{1}((g, n)) \rightarrow B_{1}((g, n))$, compatible with $\mathbf{S}_{n}$-actions.

In effect, any $\Gamma$-collection $A_{1}$ is determined up to an isomorphism by its restriction to the category of modular corollas with flags $\{1, \ldots, n\}$ and a vertex labeled by $g$. Their isomorphisms correspond to permutations of $\{1, \ldots, n\}$.

Such collections are called stable $\mathbf{S}$-modules in [GeKa2], (2.1).

b) Let $\Gamma$ be a category of oriented graphs, containing all oriented corollas. Then the category $\Gamma \mathcal{G}$-collections is equivalent to the category of double sequences $A_{1}(m, n)$ of objects in $\mathcal{G}$ endowed with actions of $\mathbf{S}_{m} \times \mathbf{S}_{n}$, and equivariant componentwise isomorphisms.

This is clear: look at oriented corollas with inputs $\{1, \ldots, n\}$ and outputs $\{1, \ldots, m\}$ : see e.g. [Va1], 1.1.

1.5.3. White product of collections. For two collections $A_{1}, B_{1}$, define their white product by

$$
\left(A_{1} \bigcirc B_{1}\right)(\sigma):=A_{1}(\sigma) \otimes B_{1}(\sigma)
$$

for any $\Gamma$-corolla $\sigma$, with obvious extension to morphisms. 
This determines a symmetric monoidal structure $\bigcirc$ on $\Gamma \mathcal{G} C O L L$.

If $(\mathcal{G}, \otimes)$ is endowed with a unit object $u$, then the collection $U, U(\sigma)=u$, sending each morphism of corollas to $i d_{u}$ is a unit object of $(\Gamma \mathcal{G C O L L}, \bigcirc)$.

1.5.4. Inner cohomomorphisms for collections. If $(\mathcal{G}, \otimes)$ admits internal cohom objects, the same holds for $(\Gamma \mathcal{G} C O L L, \bigcirc)$ : just work componentwise.

1.5.5. Endofunctor $\mathcal{F}$ on $\Gamma \mathcal{G} C O L L$. Let $A_{1}$ be a $\Gamma \mathcal{G}$-collection. Consider a $\Gamma$-corolla $\sigma$ and denote by $\Rightarrow \sigma$ the category whose objects are $\Gamma$-morphisms $\Gamma$ graphs $\tau \rightarrow \sigma$, and whose morphisms are $\Gamma$-isomorphisms of morphisms identical on $\sigma$. Put

$$
\mathcal{F}\left(A_{1}\right)(\sigma):=\operatorname{colim}_{\Rightarrow \sigma} \otimes_{v \in V_{\tau}} A_{1}\left(\tau_{v}\right)
$$

The existence of appropriate colimits in $\mathcal{G}$ such that $\otimes$ is distributive with respect to them should be postulated at this stage.

In some important cases (e.g. stable modular graphs) any category $\Rightarrow \sigma$ is essentially finite (equivalent to a category with finitely many objects and morphisms). Therefore existence of finite colimits in $\mathcal{G}$ suffices.

Clearly, this construction is functorial with respect to isomorphisms of $\Gamma$-corollas so that we actually get a new collection $\mathcal{F}\left(A_{1}\right)$. Moreover, the map $A_{1} \mapsto \mathcal{F}\left(A_{1}\right)$ extends to an endofunctor of $\Gamma \mathcal{G C O L L}$.

As is well known, functor composition endows the category of endofunctors by the structure of strict monoidal category with identity.

1.5.6. Proposition. The endofunctor $\mathcal{F}$ has a natural structure of a triple, that is, a monoid with identity in the category of endofunctors.

Proof (sketch). The argument is essentially the same as in (2.17) of [GeKa2]. We have to construct a multiplication morphism $\mu: \mathcal{F} \cdot \mathcal{F} \rightarrow \mathcal{F}$, an identity morphism $\eta: \operatorname{Id} \rightarrow \mathcal{F}$, and to check the commutativity of several diagrams.

In other words, for a variable collection $A_{1}$, we need functorial morphisms of collections $\mu_{A_{1}}: \mathcal{F}^{2}\left(A_{1}\right) \rightarrow \mathcal{F}\left(A_{1}\right), \eta_{A_{1}}: A_{1} \rightarrow \mathcal{F}\left(A_{1}\right)$ fitting the relevant commutative diagrams. In turn, to define them, we have to give their values (in $\mathcal{G}$ ) on any $\Gamma$-corolla $\sigma$, functorially with respect to $\sigma$.

The construction of $\mu$ essentially uses the hereditary property $1.3(\mathrm{vi})$ of the category $\Gamma$. In fact, we have

$$
\begin{gathered}
\mathcal{F}^{2}\left(A_{1}\right)(\sigma)=\operatorname{colim}_{\tau \rightarrow \sigma} \otimes_{v \in V_{\tau}} \mathcal{F}\left(\tau_{v}\right)= \\
\operatorname{colim}_{\tau \rightarrow \sigma}\left[\operatorname{colim}_{\rho_{v} \rightarrow \tau_{v}} \otimes_{w \in V_{\rho_{v}}} A_{1}\left(\rho_{v, w}\right)\right]
\end{gathered}
$$


where $\rho_{v}$ are objects of $\Gamma$, and $\rho_{v, w}$ is the $\Gamma$-corolla of a vertex $w$ of $\rho_{v}$. Using heredity, we can produce from each family $\rho_{v} \rightarrow \tau_{v}$ a morphism $\rho \rightarrow \tau$, and replace the right hand side of (1.5) by

$$
\operatorname{colim}_{\rho \rightarrow \tau \rightarrow \sigma} \otimes_{w \in V_{\rho}} A_{1}\left(\rho_{w}\right)
$$

The latter colimit maps to $\mathcal{F}\left(A_{1}\right)(\sigma)$ via composition of two arrows in $\rho \rightarrow \sigma$.

As Getzler and Kapranov suggest, this construction and various similar ones needed to produce $\eta_{A_{1}}$ and to check axioms, become more transparent if one uses the simplicial formalism.

Given $\sigma$ and $k \geq 0$, define the category $\Rightarrow_{k} \sigma$ : its objects are sequences of morphisms $\left(f_{1}, \ldots, f_{k}\right)$ in $\Gamma, f_{1}: \tau_{0} \rightarrow \tau_{1}, \ldots, f_{k}: \tau_{k-1} \rightarrow \tau_{k}$ together with an augmentation morphism $\tau_{k} \rightarrow \sigma$. Morphisms in $\Rightarrow_{k} \sigma$ are isomorphisms of such sequences compatible with augmentation.

Categories $\Rightarrow_{k} \sigma$ are interconnected by the standard face and degeneracy functors turning them into components of a simplicial category.

Namely, $d_{i}: \Rightarrow_{k} \sigma \rightarrow \Rightarrow_{k-1} \sigma$ skips $\tau_{0}, f_{1}$ (resp. $\left.f_{k}, \tau_{k}\right)$ for $i=0$ (resp. $i=k$ ); skips $\tau_{i}$ and composes $f_{i}, f_{i+1}$ for $1 \leq i \leq k-1$.

Similarly, $s_{i}: \Rightarrow_{k} \sigma \rightarrow \Rightarrow_{k+1} \sigma$ inserts $i d: \tau_{i} \rightarrow \tau_{i}$.

An argument similar to one which we sketched above for $k=1$ will convince the reader that one can identify the value of the $(k+1)$-th iteration $\mathcal{F}^{k+1}\left(A_{1}\right)$ at $\sigma$ with the functor sending $\sigma$ to

$$
\operatorname{colim}_{\Rightarrow_{k} \sigma} \otimes_{v \in V_{\tau_{0}}} A_{1}\left(\tau_{0, v}\right)
$$

Thus the multiplication $\mu$ is the functor induced on colimits by $d_{1}: \Rightarrow_{1} \sigma \rightarrow$ $\Rightarrow_{0} \sigma$. Monoidal identity maps $A_{1}$ to $\mathcal{F}\left(A_{1}\right)$ by sending $A_{1}(\sigma)$ to the diagram id : $A_{1}(\sigma) \rightarrow A_{1}(\sigma)$.

Two morphisms $\mathcal{F}^{3} \rightarrow \mathcal{F}^{2}$ corresponding to two configurations of brackets are induced by $d_{1}$ and $d_{2}$ respectively, and the associativity is expressed by the simplicial identity $d_{1} d_{1}=d_{1} d_{2}$. Identity $\eta$ is treated similarly.

1.6. $\Gamma \mathcal{G}$-operads as functors. Traditional approaches to operads in our context lead to three different but equivalent definitions of it. Very briefly, they can be summarized as follows.

(I) An operad is a tensor functor $(\Gamma, \coprod) \rightarrow(\mathcal{G}, \otimes)$ satisfying certain additional constraints. 
(II) An operad is a collection together with a structure of an algebra over the triple $(\mathcal{F}, \mu, \eta)$.

(III) An operad is a collection together with a composition law which makes it a monoid with respect to an appropriate symmetric monoidal structure upon $\Gamma \mathcal{G C O L L}$ (to be described).

We will start with the first description, and proceed to the second one in Sec. 1.7. As for the third description which requires first construction of a special monoidal structure on collections, it seems to be less universal. In the Appendix, we will sketch it for orientation labeling and directed graphs, following [Va1].

Consider the category whose objects are functors $A: \Gamma \rightarrow \mathcal{G}$ compatible with the monoidal structures $\coprod$ and $\otimes$ in the following sense: we are given functorial isomorphisms

$$
a_{\sigma, \tau}: A(\sigma \coprod \tau) \rightarrow A(\sigma) \otimes A(\tau)
$$

for all $\sigma, \tau \in \Gamma$ such that inverse isomorphisms $a_{\sigma, \tau}^{-1}$ satisfy conditions spelled out in Def. 1.8 of [DeMi]. Such functors form a category, morphisms in which are functor morphisms compatible with $a_{\sigma, \tau}$.

In $[\mathrm{DeMi}]$ such functors are called tensor functors, we will also use this terminology.

1.6.1. Definition. The category $\Gamma \mathcal{G O P E R}$ of $\Gamma \mathcal{G}$-operads is the category of those tensor functors $(A, a): \Gamma \rightarrow \mathcal{G}$ that send any grafting morphism, in particular $\circ_{\tau}$, in $\Gamma$ to an isomorphism.

Morphisms are functor morphisms.

Informally, making grafting morphisms invertible means that $A(\tau)$ for any $\tau \in \Gamma$ can be canonically identified with the tensor product $\otimes_{v} A\left(\tau_{v}\right)$ where $\tau_{v}$ runs over $\Gamma$-corollas of all vertices of $\tau$ (or rather, of $\psi(\tau)$ ). To see this, one should apply (1.6) (functorially extended to disjoint unions of arbitrary finite families) to the l.h.s. of (1.1).

Moreover, the morphism (1.1) of "full contraction followed by a merger", $\circ_{\tau}$ : $\tau \rightarrow \operatorname{con}(\tau)$ in $\Gamma$, combined with just described tensor decomposition produces a morphism in $\mathcal{G}$

$$
\otimes_{v \in V_{\tau}} A\left(\tau_{v}\right) \rightarrow A(\operatorname{con}(\tau)) .
$$

This is our embodiment of operadic compositions.

We will omit $a$ in the notation $(A, a)$ for brevity, and simply treat (1.6) as identical map, as well as its extensions to arbitrary families and their disjoint unions. 
1.7. From functors to algebras over the triple $(\mathcal{F}, \mu, \eta)$. Let now $A$ : $\Gamma \rightarrow \mathcal{G}$ be an operad. Denote by $A_{1}$ its restriction to the subcategory of $\Gamma$-corollas and their isomorphisms. Let $\sigma$ be a $\Gamma$-corolla. We can treat any object $\tau \rightarrow \sigma$ in $\Rightarrow \sigma$ (cf. 1.5.5) as a morphism in $\Gamma$ and apply to it the functor $A$. We will get a morphism $\otimes_{v \in V_{\tau}} A_{1}\left(\tau_{v}\right) \rightarrow A_{1}(\sigma)$ functorial with respect to isomorphisms of $\tau$ identical on $\sigma$. Due to the universal property of colimits, these morphisms induce a morphism in $\mathcal{G}$

$$
\mathcal{F}\left(A_{1}\right)(\sigma) \rightarrow A_{1}(\sigma)
$$

The system of these morphisms is functorial in $\sigma$, so we get finally a morphism of collections

$$
\alpha_{A_{1}}: \mathcal{F}\left(A_{1}\right) \rightarrow A_{1} .
$$

Similarly, among the objects of $\Rightarrow \sigma$ there is the identical morphism $i d: \sigma \rightarrow \sigma$. As above, it produces a morphism of collections

$$
\eta_{A_{1}}: A_{1} \rightarrow \mathcal{F}\left(A_{1}\right)
$$

1.7.1. Proposition. (i) The data $\left(A_{1}, \alpha_{A_{1}}, \eta_{A_{1}}\right)$ constitute an algebra over the triple $(\mathcal{F}, \mu, \eta)$ (see e.g. [MarShSt], pp. 88-89).

(ii) The map $A \mapsto\left(A_{1}, \alpha_{A_{1}}, \eta_{A_{1}}\right)$ extends to a functor coll establishing equivalence of the category of $\Gamma \mathcal{G}$-operads and the category of algebras over the triple $(\mathcal{F}, \mu, \eta)$.

Proof (sketch). The proof is essentially the same as that of Proposition (2.23) in $[\mathrm{GeKa} 2]$. The first statement reduces to the check of commutativity of several diagrams.

The main problem in the second statement consists in extending each morphism of operads as algebras to a morphism of operads as functors. In other words, knowing the operadic compositions induced by full contractions and mergers (if the latter occur in $\Gamma$ ), we want to reconstruct operadic compositions induced by partial contractions such as $\tau \rightarrow \sigma$ in (a $\Gamma$-version of) a diagram (1.3). To this end, complete (1.3) by a morphism

$$
f: \coprod_{w \in V_{\tau}} \tau_{w} \rightarrow \coprod_{v \in V_{\sigma}} \tau_{v}
$$

which is the disjoint union of morphisms of total graftings with targets $\tau_{v}$ (so that $\tau_{w}$ are corollas whereas $\tau_{v}$ generally are not). Since $k \cdot f$ is a total grafting with 
target $\tau$, in order to calculate the value of our functor on $\tau \rightarrow \sigma$ it suffices to know its value on the composition $\circ_{\sigma}\left(\coprod h_{v}\right) f$ which involves only graftings, full contractions and mergers of corollas.

1.7.2. Algebra $\mathcal{F}\left(A_{1}\right)$. As a general formalism shows, for any $A_{1}$, the collection $\mathcal{F}\left(A_{1}\right)$ has the canonical structure of an operad, with $\alpha_{\mathcal{F}\left(A_{1}\right)}:=\mu_{A_{1}}: \mathcal{F}^{2}\left(A_{1}\right) \rightarrow$ $\mathcal{F}\left(A_{1}\right)$.

1.7.3. Proposition. For any collection $A_{1}$ and an operad $B$, there exists a canonical identification

$$
\operatorname{Hom}_{\Gamma \mathcal{G C O L L}}\left(A_{1}, \operatorname{coll}(B)\right)=\operatorname{Hom}_{\Gamma \mathcal{G O P E R}}\left(\mathcal{F}\left(A_{1}\right), B\right)
$$

functorial in both arguments.

This means that the functor $\mathcal{F}: \Gamma \mathcal{G} C O L L \rightarrow \Gamma \mathcal{G O P E R}$ is a construction of the free operad freely generated by a collection, and is thus an analog of tensor algebra of a linear space.

1.8. White product of operads. For two $\Gamma \mathcal{G}$-operads $A, B$, define their white product by

$$
(A \bigcirc B)(\sigma):=A(\sigma) \otimes B(\sigma)
$$

for any object $\sigma \in \Gamma$. The extension to morphisms is evident.

This determines a symmetric monoidal structure $\bigcirc$ on $\Gamma \mathcal{G O P E R}$.

As before, if $(\mathcal{G}, \otimes)$ is endowed with a unit object $u$, then the functor $U: \sigma \mapsto u$ sending each morphism of labeled graphs to $i d_{u}$ is a unit object of $(\Gamma \mathcal{G O P E R}, \bigcirc)$.

1.9. Morphism $j$. Morphism of operads $j: \mathcal{F}\left(E_{1} \bigcirc B_{1}\right) \rightarrow \mathcal{F}\left(E_{1}\right) \bigcirc \mathcal{F}\left(B_{1}\right)$ that is, a family of morphisms

$\operatorname{colim}_{\Rightarrow \sigma} \otimes_{v \in V_{\tau}} E_{1}\left(\tau_{v}\right) \otimes B_{1}\left(\tau_{v}\right) \rightarrow\left(\operatorname{colim}_{\Rightarrow \sigma} \otimes_{v \in V_{\tau^{\prime}}} E_{1}\left(\tau_{v}{ }^{\prime}\right)\right) \otimes\left(\operatorname{colim}_{\Rightarrow \sigma} \otimes_{v \in V_{\tau^{\prime \prime}}} B_{1}\left(\tau_{v}{ }^{\prime \prime}\right)\right)$

comes from the "diagonal" part of the right hand side: $\tau=\tau^{\prime}=\tau^{\prime \prime}$.

\section{$\S 2$. Inner cohomomorphism operads}

2.1. Preparation. Fix a graph category $\Gamma$ and a ground category $\mathcal{G}$ as above.

In this Section, we will assume that $(\mathcal{G}, \otimes)$ has inner cohomomorphism objects. Moreover, for validity of the main Theorem $2.2, \mathcal{G}$ must be abelian, with tensor product exact in each argument. 
Let $A$ be a $\Gamma \mathcal{G}$-operad, $A_{1}$ a $\Gamma \mathcal{G}$-collection, and $i_{A}: A_{1} \rightarrow A$ a morphism of collections such that the respective morphism of operads $f_{A}: \mathcal{F}\left(A_{1}\right) \rightarrow A$ is surjective. Denote by $\mathcal{A}$ the diagram $i_{A}: A_{1} \rightarrow A$. Such a diagram can be thought of as a presentation of $A$.

Let $\mathcal{B}$ be a similar data $i_{B}: B_{1} \rightarrow B$.

As in 0.1 , denote by $\mathcal{A} \Rightarrow \mathcal{B}$ the category whose objects are commutative diagrams

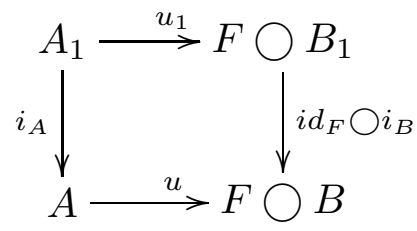

where $F$ is an operad, $u$ is a morphism of operads, and $u_{1}$ is a morphism of collections. In the upper row, and below in similar situations, we write $F$ in place $\operatorname{coll}(F)$ for brevity, identifying an operad with its underlying collection.

Notice that, unlike in 0.1 , we do not assume that $i_{A}, i_{B}$ are injective. Hence the upper row of (2.1) has to be given explicitly.

Such a diagram can be denoted $\left(F, u, u_{1}\right)$ since the remaining data are determined by $\mathcal{A}, \mathcal{B}$.

A morphism $\left(F, u, u_{1}\right) \rightarrow\left(F^{\prime}, u^{\prime}, u_{1}^{\prime}\right)$ in $\mathcal{A} \Rightarrow \mathcal{B}$ is a morphism of operads $F \rightarrow F^{\prime}$ inducing a morphism of commutative diagrams (2.1) constructed for $F$ and $F^{\prime}$ respectively.

2.2. Theorem. The category $\mathcal{A} \Rightarrow \mathcal{B}$ has an initial object

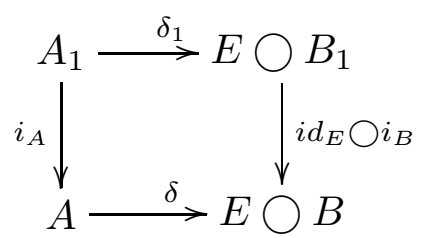

defined uniquely up to unique isomorphism.

Moreover, $E$ comes together with a presentation $\mathcal{E}, i_{E}: E_{1} \rightarrow E$ in which $E_{1}=\underline{\operatorname{cohom}}\left(A_{1}, B_{1}\right)$, inner cohomomorphism being taken in the category of $\Gamma \mathcal{G}_{-}$ collections.

If $F$ is given together with its presentation $\mathcal{F}$, that is $i_{F}: F_{1} \rightarrow F$, and $u$ is induced by $u_{1}: A_{1} \rightarrow F_{1} \bigcirc B_{1}$, then the canonical homomorphism $E \rightarrow F$ is induced by a unique morphism in the category $\mathcal{E} \Rightarrow \mathcal{F}$. 
Proof. (i) Preparation. The morphism $u_{1}: A_{1} \rightarrow F \bigcirc B_{1}$ corresponds to a morphism $\tilde{u}_{1}: E_{1} \rightarrow F$ where $E_{1}=\underline{\operatorname{cohom}}\left(A_{1}, B_{1}\right)$ as above. Recall that inner cohomomorphism collections here can be constructed componentwise. Let $c: A_{1} \rightarrow E_{1} \bigcirc B_{1}$ be the coevaluation morphism. Then the diagram

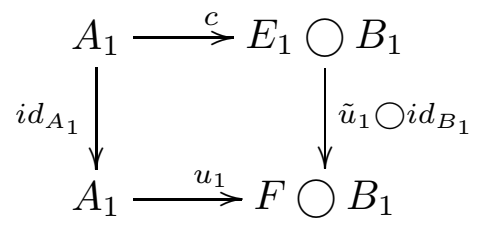

is commutative. Composing (2.3) with (2.1), we get a commutative square of collections

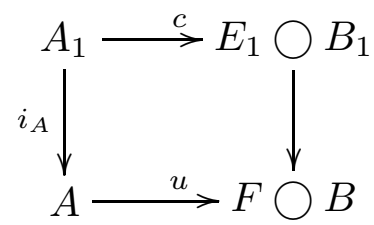

which produces a morphism of operads with presentations

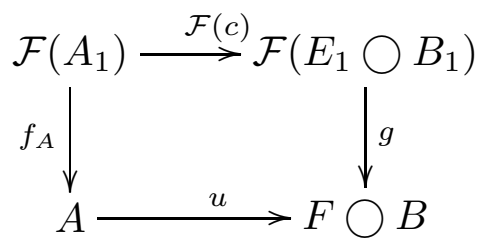

This diagram can be completed by the commutative triangle

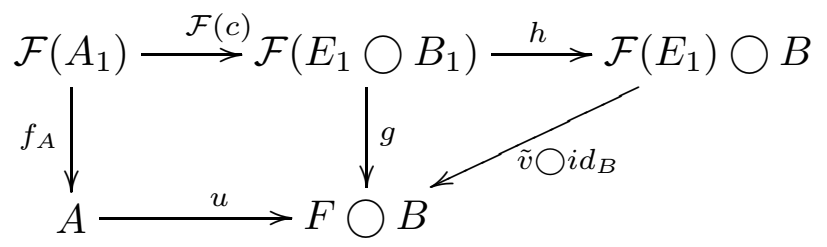

where $h$ is the composition

$$
\mathcal{F}\left(E_{1} \bigcirc B_{1}\right) \stackrel{j}{\longrightarrow} \mathcal{F}\left(E_{1}\right) \bigcirc \mathcal{F}\left(B_{1}\right) \stackrel{i d \bigcirc f_{B}}{\longrightarrow} \mathcal{F}\left(E_{1}\right) \bigcirc B
$$

and $j$ is described in Section 1.9. 
(ii) Construction of main objects. Now we will construct in turn an ideal $(\tilde{R}) \subset$ $\mathcal{F}\left(E_{1}\right)$, the operad $E:=\mathcal{F}\left(E_{1}\right) /(\tilde{R})$ together with a morphism of operads $\delta: A \rightarrow$ $E \bigcirc B$ and a morphism $v: E \rightarrow F$.

Starting with this point, we will have to use our assumption that assume that $\mathcal{G}$ is abelian, and $\otimes$ is exact.

Choose a subcollection $R \subset \operatorname{Ker} f_{A}$ generating $\operatorname{Ker} f_{A}$ as an ideal in the free operad $\mathcal{F}\left(A_{1}\right)$. Replace the morphism $h \circ \mathcal{F}(c): \mathcal{F}\left(A_{1}\right) \rightarrow \mathcal{F}\left(E_{1}\right) \bigcirc B$ by the morphism canonically corresponding to it

$$
\underline{\operatorname{cohom}}\left(\mathcal{F}\left(A_{1}\right), B\right) \rightarrow \mathcal{F}\left(E_{1}\right)
$$

where the inner cohomomorphisms here and below are taken in the category of collections.

Since inner cohomomorphisms are covariant functorial with respect to the first argument, we have a commutative diagram

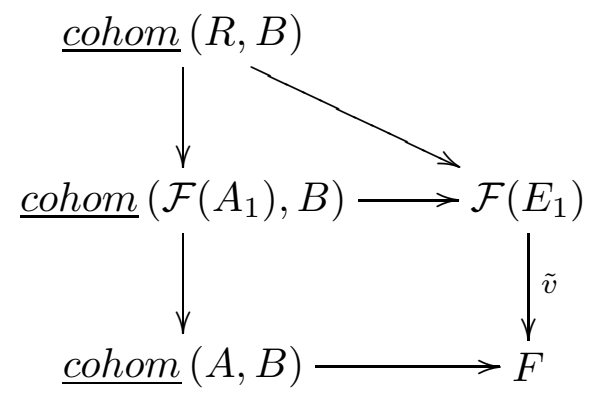

where $\tilde{v}$ is taken from $(2.6)$.

Denote by $\tilde{R}$ the image of the skew arrow in (2.7). Since the composition $R \rightarrow$ $\mathcal{F}\left(A_{1}\right) \rightarrow A$ is zero, the same holds for the composition of the two left vertical arrows in (2.7). This implies that the composition $\tilde{R} \rightarrow \mathcal{F}\left(E_{1}\right) \rightarrow F$ is zero. Since $\tilde{v}$ is a morphism of operads, its kernel contains the ideal $(\tilde{R})$ generated by $\tilde{R}$.

Rewrite the upper triangle in (2.7) as a commutative square

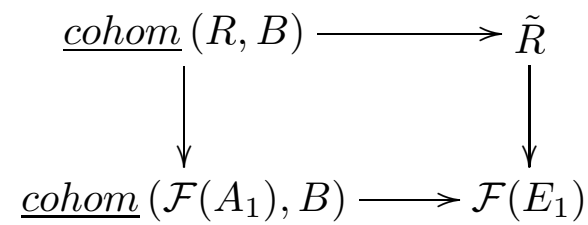


Replacing the horizontal arrows with the help of coevaluation morphisms, we get the commutative square

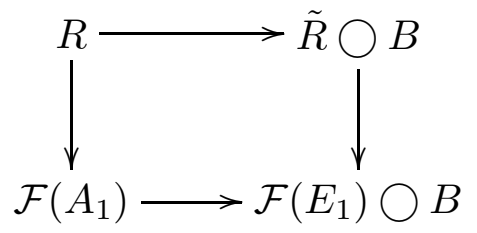

which induces a morphism of operads

$$
\delta: A=\mathcal{F}\left(A_{1}\right) /(R) \rightarrow \mathcal{F}\left(E_{1}\right) \bigcirc B /(\tilde{R} \bigcirc B) \cong \mathcal{F}\left(E_{1}\right) /(\tilde{R}) \bigcirc B=E \bigcirc B
$$

(use the exactness of $\otimes$ in $\mathcal{G}$.)

(iii) Completion of the proof. It remains to show that $\delta: A \rightarrow E \bigcirc B$ has all the properties stated in Theorem 2.2.

From our construction, it is clear that it fits into the diagram of the form (2.2), and that it comes with the presentation $i_{E}: E_{1} \rightarrow E$.

The morphism $\tilde{v}: \mathcal{F}\left(E_{1}\right) \rightarrow F$ descends to a morphism of operads $v: E \rightarrow F$, and from the commutativity of the diagram (2.6) one can infer that it induces a morphism of objects $\left(E, \delta, \delta_{1}\right) \rightarrow\left(F, u, u_{1}\right)$.

We leave the remaining checks to the reader.

2.3. Remark. Associative algebras (without unit) can be treated as operads: functors on linear oriented trees and contractions with values in linear spaces. The Theorem 2.2 in this case reduces to the Theorem 0.1 .1, or rather its extension where presentations are not supposed to be injective on components of degree 1.

2.4. Inner cohomomorphisms for operads with presentation. We can now reformulate Theorem 2.2 in the same way as it was done in the Introduction for associative algebras.

Consider the following category $O P$ of $\Gamma \mathcal{G}$ operads given together with their presentation.

Objects of $O P$ are pairs $\mathcal{A}$ as in 2.1. A morphism $\mathcal{A} \rightarrow \mathcal{B}$ is a pair consisting of a morphism of collections $A_{1} \rightarrow B_{1}$ and a morphism of operads $A \rightarrow B$ compatible with $i_{A}, i_{B}$.

This category has a symmetric monoidal product $\odot$ induced by $\bigcirc$ in the following sense: $\mathcal{A} \odot \mathcal{B}=\mathcal{C}$ where $\mathcal{C}$ is represented by $C_{1}:=A_{1} \bigcirc B_{1}, C:=$ the minimal 
suboperad containing the image $\left(i_{A} \bigcirc i_{B}\right)\left(C_{1}\right)$ in $A \bigcirc B$, and $i_{C}:=$ restriction of $i_{A} \bigcirc i_{B}$

Theorem 2.2 can now be read as a statement that, functorially in all arguments, we have

$$
\operatorname{Hom}_{O P}(\mathcal{A}, \mathcal{F} \bigodot \mathcal{B})=\operatorname{Hom}_{O P}(\mathcal{E}, \mathcal{F})
$$

that is, $\mathcal{E}$ is an inner cohomomorphism object in $O P$ :

$$
\mathcal{E}=\underline{\operatorname{cohom}}_{O P}(\mathcal{A}, \mathcal{B})
$$

General categorical formalism produces canonical comultiplication morphisms in $O P$

$$
\Delta_{\mathcal{A}, \mathcal{B}, \mathcal{C}}: \underline{\operatorname{cohom}}_{O P}(\mathcal{A}, \mathcal{C}) \rightarrow \underline{\operatorname{cohom}}_{O P}(\mathcal{A}, \mathcal{B}) \bigodot \underline{\operatorname{cohom}}_{O P}(\mathcal{B}, \mathcal{C})
$$

coassociative in an evident sense.

This is an operadic version of quantum matrices and their comultiplication.

In particular, the operad

$$
\underline{\operatorname{coend}}_{O P} \mathcal{A}:=\underline{\text { cohom }}_{O P}(\mathcal{A}, \mathcal{A})
$$

is endowed with a canonical coassociative comultiplication, morphism of operads

$$
\Delta_{\mathcal{A}}:=\Delta_{\mathcal{A}, \mathcal{A}, \mathcal{A}}: \underline{\operatorname{coend}}_{O P} \mathcal{A} \rightarrow \underline{\operatorname{coend}}_{O P} \mathcal{A} \bigodot \underline{\operatorname{coend}}_{O P} \mathcal{A}
$$

It is generally not cocommutative, as the case of associative rings amply demonstrates.

We get thus a supply of "quantum semigroups", or Hopf algebras in the category of operads (ignoring antipode).

Notice finally that if $\mathcal{G}$ has a unit object $u$, we can sometimes define a unit object $U$ in $(O P, \odot)$. Taking $\mathcal{F}=U$ in the adjunction formula (2.10), we see that the space of "classical homomorphisms" $\mathcal{A} \rightarrow \mathcal{B}$ in $O P$ coincides with space of points of $\mathcal{E}$ with values in $U$ (here we implicitly imagine operadic affine quantum spaces as objects of the dual category).

2.5. Algebras over an operad and their deformations. For certain labelings and respective graph categories $\Gamma$ one can define a class of "natural" $\Gamma \mathcal{G}-$ collections. 
The basic example is this. Let $J$ be an abstract set of "flavors" such that a labeling of $\tau$ consists of a map $F_{\tau} \rightarrow J$ (and possibly other data). (Imagine orientations, colors, or pairs (orientation, color)).

Assume furthermore that automorphisms of any $\Gamma$-corolla $\sigma$ form a subgroup of permutations of its flags preserving flavors of flags. In this case any family of objects $\mathbf{V}:=\left\{V_{j} \mid j \in J\right\}$ determines the following $\Gamma$-collection:

$$
\operatorname{Coll}(\mathbf{V})(\sigma):=\otimes_{j \in J} V_{j}^{\otimes F_{\tau}^{(j)}}
$$

where $F_{\tau}^{(j)}$ is the subset of flags of flavour $j$. Automorphisms of $\sigma$ act in an evident way.

Such a collection naturally extends to a functor on the groupoid of $\Gamma$-graphs $\tau$ and their isomorphisms: simply replace $\sigma$ by $\tau$ in (2.14).

In order to extend it to arbitrary $\Gamma$-morphisms, consider separately three classes of morphisms.

a) Graftings. Graftings correspond to bijections of sets of flags preserving flavors. Hence they extend to (2.14).

b) Mergers. They have the same property.

c) Contractions. In principle, in order to accommodate contractions, we have to impose on $\mathbf{V}$ an additional structure, namely, a set of polylinear forms on $\left(V_{j}\right)$ which we denote $\mathbf{v}$ and axiomatize as follows:

Let $S$ be a finite set and $\kappa:=\left\{\left(j_{s}, k_{s}\right) \mid s \in S\right\}$ be a family of pairs of flavors such that in some $\Gamma$-graph there exist two vertices (perhaps coinciding) and connecting them edges which are simultaneously contracted by a $\Gamma$-morphism. The respective component $v_{\kappa}$ of $\mathbf{v}$ is a morphism in $\mathcal{G}$

$$
v_{\kappa}: \otimes_{s \in S}\left(V_{j_{s}} \otimes V_{k_{s}}\right) \rightarrow u
$$

where $u$ is the unit object of $\mathcal{G}$.

Given $\mathbf{v}$, the prescription for extending our functor to contractions looks as follows. To map a product (2.14) for the source of a contraction to the similar product for its target, we must map identically factors corresponding to uncontracted flags, and to "kill" factors of the type $\otimes_{s \in S} V_{j_{s}} \otimes V_{k_{s}}$ with the help of (2.15).

This prescription will not necessarily describe a functor $\Gamma \rightarrow \mathcal{G}$ : one should to impose upon $\mathbf{v}$ coherence conditions which we do not bother to spell out here. 
In real life, this problem is avoided by specifying only bilinear forms corresponding to one-edge contractions, and then tensoring them to obtain full scale (2.15).

We can now give the main definition of this Section.

2.5.1. Definition. a) Any functor as above $\Gamma \rightarrow \mathcal{G}$ with underlying collection Coll $(\mathbf{V})$ and structure forms $\mathbf{v}$ is called the endomorphism operad of $(\mathbf{V}, \mathbf{v})$ and denoted OpEnd $(\mathbf{V}, \mathbf{v})$.

b) Let $\mathcal{P}$ be a $\Gamma \mathcal{G}$-operad. Any morphism $P \rightarrow O p E n d(\mathbf{V}, \mathbf{v})$ is called a structure of $P$-algebra on $(\mathbf{V}, \mathbf{v})$.

Examples. We will illustrate this on three types of labelling discussed in 1.3.2 above.

a) Working with a subcategory of $G r$ itself (as in the case of cyclic operads) we should choose one object $V$ of $\mathcal{G}$ and a symmetric pairing $g: V \otimes V \rightarrow u$.

b) Let now $\Gamma$ be a subcategory of oriented graphs. In that case one usually chooses $V_{\text {out }}=V, V_{\text {in }}=V^{t}$ (the dual object), and takes for $v$ the canonical pairing $V^{t} \otimes V \rightarrow u$.

c) Finally, let $\Gamma$ be a category of colored graphs, $I$ the set of colors. In this case, one applies the full machinery of the definition above, simplifying it by caring only about one-edge contractions. So we need $V_{i} \otimes V_{i} \rightarrow u$ in the unoriented case, or else choose $V_{\text {out }, i}=V_{i n, i}^{t}$.

Let us now return to our definition 2.5.1. The whole space of structures of $P$-algebra on $(\mathbf{V}, \mathbf{v})$ is thus

$$
\operatorname{Hom}_{\Gamma \mathcal{G O P E R}}(P, \operatorname{OpEnd}(\mathbf{V}, \mathbf{v})) \text {. }
$$

In the standard approach to the deformation theory of operadic algebras one chooses a space of basic operations, that is, a presentation of $P$ (and then replaces the respective structure by a differential in an appropriate Hochschild-type complex, the step that we will not discuss here). Sometimes, one can choose a compatible presentation of $O p E n d(\mathbf{V}, \mathbf{v})$. For example, if connected graphs in $\Gamma$ consist of oriented trees and all basic operations are binary, one can choose for generators the collection $P_{1}$ which coincides with $P$ on corollas with three flags and is zero otherwise. Respectively, $O p E n d V$ is (hopefully) generated by $\underline{h o m}\left(V^{\otimes 2}, V\right)$ (at least, this is the case for $\mathcal{G}=V e c_{k}$.)

Accepting this, we suggest to replace $P$ by $\mathcal{P}$ which is $i: P_{1} \rightarrow P$, to augment $O p E n d V$ accordingly, and to replace $(2.16)$ by an appropriate set of morphisms in 
the category $O P$. After that, it is only natural to consider the operad

$$
\underline{\operatorname{cohom}}_{O P}(\mathcal{P}, \mathcal{O} p \mathcal{E} n d V)
$$

as a non-commutative space parameterizing deformations of (a chosen collection of generators of) $P$-algebra structures.

2.6. Inner cohomomorphisms for algebras over an operad. $P$-algebras form a category $P A L G$. One can try to play with $P$-algebras the same game as we did with associative algebras and operads of various types, and to study existence of inner cohomomorphisms in a category of $P$-algebras with a presentation. However, even in order to state the problem we need at least two preliminary constructions:

a) Free $P$-algebra $F_{P}(\mathbf{V}, \mathbf{v})$ generated by a family $(\mathbf{V}, \mathbf{v})$ as above. Generally, it will take values in a monoidal category larger than $\mathcal{G}$, e.g. that of inductive systems.

This will allow us to define the notion of an algebra with presentation.

b) Symmetric white product on the category of $P$-algebras extending $\otimes$.

To this end, we need a symmetric comultiplication $\Delta: P \rightarrow P \bigcirc P$ and an analog of the morphism $j$ from Sec. 1.9 for free $P$-algebras.

Unfortunately, as our description of $P$-algebras above shows, we cannot do even this preliminary work in the same generality as we treated operads themselves. Therefore we step back, and for the remainder of Section 2.6 work with a version of ordinary operads.

2.6.1. Ordinary operads and their algebras. Let $\Gamma$ be the category of graphs whose connected components are directed trees with exactly one output and at least one input at each vertex. Morphisms are contractions and graftings; mergers are not allowed. Let $(\mathcal{G}, \otimes, u)$ be an abelian symmetric monoidal category, such that $\otimes$ is exact in both arguments, and endowed with finite colimits and cohomomorphism objects.

Consider a $\Gamma \mathcal{G}$ operad $P$. Denote by $P(n)$ the value of $P$ on the $\Gamma$-corolla with inputs $\{1, \ldots, n\}$, and let $\mathbf{S}_{n}$ be the automorphism group of this directed corolla. Assume that $P(1)=u$ and that contracting an edge one end of which carries a corolla with one input produces canonical identifications $Q \otimes u \rightarrow Q$.

It is well known that a free $P$-algebra freely generated by $V_{1}$ exists in an appropriate category of inductive limits, and its underlying object is

$$
F_{P}\left(V_{1}\right)=\oplus_{n=1}^{\infty} P(n) \otimes \mathbf{s}_{n} V_{1}^{\otimes n}
$$


This construction is functorial in $V_{1}$. A presentation of an algebra $V$ is a surjective morphism $F_{P}\left(V_{1}\right) \rightarrow V$; it can be reconstructed from its restriction $i_{V}: V_{1} \rightarrow V$.

Assume that $P$ is endowed with a symmetric comultiplication $\Delta: P \rightarrow P \bigcirc P$.

Then, given two objects $E_{1}, W_{1}$ of $\mathcal{G}$, we can define a map

$$
j: F_{P}\left(E_{1} \otimes W_{1}\right) \rightarrow F_{P}\left(E_{1}\right) \bigcirc F_{P}\left(W_{1}\right) .
$$

To construct it, first produce for each $n$ a map

$$
P(n) \otimes\left(E_{1} \otimes W_{1}\right)^{\otimes n} \rightarrow P(n) \otimes E_{1}^{\otimes n} \otimes P(n) \otimes W_{1}^{\otimes n}
$$

combining $\Delta$ with regrouping, and then the induced map of colimits

$$
j(n): P(n) \otimes \mathbf{S}_{n}\left(E_{1} \otimes W_{1}\right)^{\otimes n} \rightarrow\left(P(n) \otimes \mathbf{S}_{n} E_{1}^{\otimes n}\right) \otimes\left(P(n) \otimes \mathbf{S}_{n} W_{1}^{\otimes n}\right) .
$$

We put $j=\oplus_{n} j(n)$. One can check that this is a morphism of $P$-algebras.

If $V, W$ are two $P$-algebras, presented by their structure morphisms $\alpha: F_{P}(V) \rightarrow$ $V, \beta: F_{P}(W) \rightarrow W$, we can compose $j(n)$ with $\alpha \otimes \beta$ to define a structure of $P-$ algebra on $V \otimes W$. This gives a symmetric monoidal structure on $P A L G$ still denoted $\otimes$. We can now state

2.6.2. Proposition. Analog of Theorem 2.2 holds in the category of P-algebras with presentation.

We skip a proof which follows the same plan as that of Theorem 2.2.

2.7. Explicit constructions of cohomomorphism objects in the category of associative algebras. Existence proof of cohomomorphism objects generally is not very illuminating. In this and the following subsections, we cite several explicit constructions, valid under additional assumptions.

a) Quadratic algebras. This was the case first treated in [Ma1] and [Ma2]. Briefly, a quadratic algebra $A$ (over a field $k$ ) is defined by its presentation $\alpha: F_{A S S}\left(A_{1}\right) \rightarrow$ $A$ where $F_{A S S}\left(A_{1}\right)=T\left(A_{1}\right)$ is the free (tensor) algebra freely generated by a finitedimensional vector space $A_{1}$, such that $\operatorname{Ker} \alpha$ is the ideal generated by the space of quadratic relations $R_{A} \subset A_{1}^{\otimes 2}$. In particular, $A_{1}$ is embedded in $A$, each $A$ is naturally graded ( $A_{1}$ in degree 1$)$, and a morphism of quadratic presentations is the same as morphism of algebras which preserves grading. Moreover, each morphism is uniquely defined by its restriction to the space of generators.

This category $Q$ alg has a contravariant duality involution: $A \mapsto A^{!}$where $A^{!}=$ $T\left(A_{1}^{*}\right) /\left(R_{A}^{\perp}\right), A_{1}^{*}$ denoting the dual space to $A_{1}$. 
It has also two different symmetric monoidal structures, which are interchanged by !: "white product" $\circ$ and "black product" •:

$$
\begin{gathered}
T\left(A_{1}\right) /\left(R_{A}\right) \circ T\left(B_{1}\right) /\left(R_{B}\right)=T\left(A_{1} \otimes B_{1}\right) /\left(S_{(23)}\left(R_{A} \otimes B_{1}^{\otimes 2}+A_{1}^{\otimes 2} \otimes R_{B}\right),\right. \\
T\left(A_{1}\right) /\left(R_{A}\right) \bullet T\left(B_{1}\right) /\left(R_{B}\right)=T\left(A_{1} \otimes B_{1}\right) /\left(S_{(23)}\left(R_{A} \otimes R_{B}\right),\right.
\end{gathered}
$$

Here $S_{(23)}: A_{1}^{\otimes 2} \otimes B_{1}^{\otimes 2} \rightarrow\left(A_{1} \otimes B_{1}\right)^{\otimes 2}$ interchanges two middle factors.

Both categories have unit objects: polynomials of one variable for $\circ$, and dual numbers $k[\varepsilon] /\left(\varepsilon^{2}\right)$ for $\bullet$ respectively. They are !-dual to each other. The generator $\varepsilon$ combined with general categorical constructions produces differential in various versions of Koszul complex which is a base of Koszul duality.

The monoidal category $($ Q alg,$\bullet)$ has internal homomorphism objects. Explicitly,

$$
\underline{h o m}_{\bullet}(A, B)=A^{!} \circ B .
$$

The monoidal category $($ Qalg, o) has inner cohomomorphism objects. Explicitly,

$$
\text { cohom }_{\circ}(A, B)=A \bullet B^{!} .
$$

Claim. (2.23) is a valid description of the internal cohomomorphism object of two quadratic algebras in the total category of (algebras with) presentations.

These formulas follow from a general functorial isomorphism (adjunction formula)

$$
\operatorname{Hom}_{Q a l g}\left(A \bullet B^{!}, C\right)=\operatorname{Hom}_{Q a l g}(A, B \circ C)
$$

Abstract properties of Qalg expressed by (2.22)-(2.24) can be axiomatized to produce an interesting version of the notion of rigid tensor category of [DeMi]. They justify the use of the name "quantum linear spaces" for objects of Qalg although the category itself is not linear or even additive.

It might be worthwhile to consider $($ Qalg,,$)$ and $(Q a l g, \bullet)$ as ground categories for $\Gamma$-operads (and cooperads).

Notice that the cohomology spaces of $\bar{M}_{0, n+1}$, components of the Quantum Cohomology cooperad, are quadratic algebras (Keel's theorem).

b) $N$-homogeneous algebras. It was shown in [BerDW] that similar results hold for the category $H_{N}$ alg of homogeneous algebras generated in degree 1 with relations generated in degree $N$, for any fixed $N \geq 2$. If one continues to denote 
by $R_{A} \subset A_{1}^{\otimes N}$ generating relations, $A^{!}$is given by relations $R_{A}^{\perp} \subset\left(A_{1}^{*}\right)^{\otimes N}$. Thus $!=!_{N}$ explicitly depends on $N$ and gives, for example, different dual objects of a free algebra, depending on where we put its trivial relations. In the definitions (2.20) one should make straightforward modifications, replacing 2 by $N$. Formulas $(2.22)-(2.24)$ still hold in the new setup. Unit object for $\bullet$ is now $k[\varepsilon] /\left(\varepsilon^{N}\right)$.

As a consequence, Koszul complexes become $N$-complexes leading to an interesting new homological effects: see [BerM] and references therein.

c) Homogeneous algebras generated in degree one. This case is treated in Chapter 3 of $[\mathrm{PP}]$ and in Section 1.3 of [GrM]; the approaches in these two papers nicely complement each other.

White product $(2.21)$ and its $H_{N} a l g$-version extend to this larger category as $(A \circ B)_{n}:=A_{n} \otimes B_{n}$ (Segre product). The duality morphism ! and the black product do not survive in $[\mathrm{PP}]$, and internal homomorphism objects (2.22) perish. The right hand side of the formula $(2.23)$ is replaced by a rather long combinatorial construction which we do not reproduce here, and (2.24) becomes simply the characteristic property of cohom's:

$$
\operatorname{Hom}(\underline{\operatorname{cohom}}(A, B), C)=\operatorname{Hom}(A, B \circ C)
$$

However, if $A$ is quadratic, then (2.23) can be resurrected in a slightly modified form: $B$ is the quotient of a quadratic algebra $\mathrm{q} B$ (leave the same generators and only quadratic relations), and we have

$$
\underline{\operatorname{cohom}}_{\circ}(A, B)=A \bullet(\mathrm{q} B)^{!}
$$

(see [PP], Ch.3, Proposition 4.3).

An important novelty of $[\mathrm{PP}]$ is a treatment of white products and cohom objects in the categories of graded modules over graded rings.

In $[\mathrm{GrM}]$, a version of ! and several versions of the black product are introduced. They are not as neatly packed together however as in the cases Qalg and $H_{N} a l g$. In particular, ! is not an involution. As a compensation, Theorem 1 of [GrM] establishes a nice extension of (2.25) for general homogeneous $A$ and $B$ involving a certain "triangle product".

A logical next step would be the introduction of these notions into "non-commutative projective algebraic geometry" where coherent sheaves appear à la Serre as quotient categories of graded modules, and cohom's of graded rings can serve as interesting non-commutative correspondences. 
2.8. Explicit constructions of cohomomorphism objects in the categories of operads. One objective of the brief review above was to collect a list of patterns that could be subsequently recognized in various categories of operads. Most of the existing results which we are aware of concern quadratic operads and Koszul duality patterns. Cohomomorphism objects appear as a byproduct, although, as we have seen above, their existence is the most persistent phenomenon, even when the neat package $(\circ, \bullet, !)$ cannot be preserved.

a) Binary quadratic operads. The pioneering paper $[\mathrm{GiKa}]$ defined $(\circ, \bullet, !)$ for binary quadratic (ordinary) operads. Their construction uses a description of operads as monoids in $(C O L L, \otimes)$, a category of collections endowed with a monoidal structure $\otimes$ (in 1.6 above, this is description (III)). This description was rather neglected here (see Appendix), but it makes clear the analogy between the tensor algebra of a linear space and the free operad generated by a collection $V$.

B. Vallette in [Va3] describes a construction of a free monoid which allows him to treat the cases when the relevant monoidal structure is not biadditive, which is the case of operads. The resulting weight grading of the free monoid can be used to define weight graded quotients, analogs of graded associative algebras: see [Fr], [Va1], [Va2].

The subcategory of ordinary operads with presentation considered in [GiKa] consists of weight graded operads generated by their binary parts (values on corollas with two inputs), with relations in weight 2 . After introducing $(\circ, \bullet, !)$, Ginzburg and Kapranov prove the adjunction formula (2.24) and thus the formula (2.23) as well.

b) Developing this technique, B. Vallette in [Va1] and [Va2] defines o for properads (see Appendix) with presentation and studies the case of (weight) quadratic relations for generators of arbitrary arity. The full adjunction formula (2.24) is established for quadratic operads with generators of one and the same arity $k \geq 2$ ([Va2], Sec. 4.6, Theorem 26), thus generalizing the $k=2$ case of [GiKa].

Yet another version of this result is proved for $k$-ary quadratic regular operads, with different definitions of black and white products ([Va2], Sec. 5.1, Theorem 40). (A regular operad is an ordinary symmetric operad which is induced by some non-symmetric operad in a sense that will not be made precise here).

\section{$\S 3$. Non-abelian constructions}

3.0. Introduction. In Sec. 2 it was proved that given an abelian category $\mathcal{G}$ with a symmetric monoidal structure $\otimes$ and its left adjoint $\underline{\text { cohom }}$, the categories 
of operads in $\mathcal{G}$ and algebras over Hopf operads also possess cohom for the natural extension of $\otimes$. In this Section we prove a version of this result in a non-abelian setting.

More precisely, we start with the description of operads as algebras over the triple $(\mathcal{F}, \mu, \eta)$, cf. Proposition 1.7.1. Here $\mathcal{F}$ is an explicit endofunctor on the category of collections $\Gamma \mathcal{G} C O L L$ which inherits from $\mathcal{G}$ a monoidal structure, an abelian structure, and inner cohomomorphisms.

We replace $\Gamma \mathcal{G} C O L L$ with an abstract monoidal category $(\mathcal{C}, \otimes)$ endowed with an endofunctor $T$ which has the structure of a triple that commutes (up to a natural transformation) with the monoidal structure. In this case, with some additional assumptions, extension of cohom is straightforward and is given by the adjoint lifting theorem. In this formulation it is unnecessary to suppose that the monoidal product is symmetric, however, when applied to operads, the triple itself is produced using symmetric properties of the monoidal structure.

Finally we formulate the natural notion of the derived cohom and consider some cases when such a functor exists.

3.1. Tensor product and cohom for algebras over a triple. It is well known that if a category is equipped with a monoidal structure distributive with respect to direct sums, then categories of algebras over ordinary Hopf operads in this category will possess an extension of the monoidal structure. We can treat more general operads considering them in the context of triples. In this Section we formulate conditions on the triples, needed to extend monoidal structure to algebras over them.

Let $(\mathcal{C}, \otimes)$ be a category with a coherently associative product (bifunctor). We do not assume $\otimes$ to be symmetric or to possess a unit object.

3.1.1. Definition. A Hopf-like triple on $\mathcal{C}$ is a triple $T: \mathcal{C} \rightarrow \mathcal{C}, \mu: T \circ T \rightarrow T$, together with a natural transformation between bifunctors on $\mathcal{C}$ :

$$
\tau: T \circ \otimes \rightarrow \otimes \circ(T \times T),
$$

satisfying the following conditions:

a) We have a commutative diagram of natural transformations:

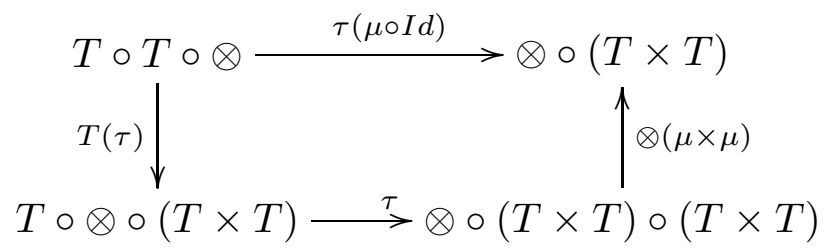


where $\mu: T^{2} \rightarrow T$ is the structure natural transformation of $T$.

b) Let $\alpha: \otimes \circ(\otimes \times I d) \rightarrow \otimes \circ(I d \times \otimes)$ be the associativity constraint for $\otimes$. Then the following diagram of natural transformations is commutative:

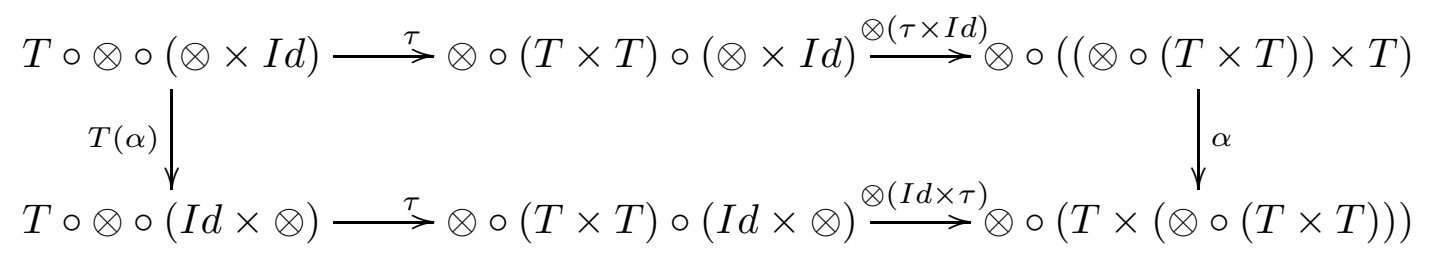

The conditions in the definition above allow us to define an associative product on the category of $T$-algebras, by extending it from $\mathcal{C}$. The functor morphism $\tau$ provides a definition, condition a) ensures that different ways of composing $\tau$ produce the same result, and condition b) implies coherent associativity of the resulting product on the category of $T$-algebras by utilizing associativity isomorphisms of $\otimes$. Here is the formal reformulation of all this.

3.1.2. Definition. Let $(A, \alpha: T(A) \rightarrow A),(B, \beta: T(B) \rightarrow B)$ be two $T$-algebras in $\mathcal{C}$. We define $(A, \alpha) \bigcirc(B, \beta)$ to be $(A \otimes B, \alpha \bigcirc \beta)$, where $\alpha \bigcirc \beta$ : $T(A \otimes B) \rightarrow A \otimes B$ is the composition

$$
T(A \otimes B) \stackrel{\tau}{\rightarrow} T(A) \otimes T(B) \stackrel{\alpha \otimes \beta}{\rightarrow} A \otimes B .
$$

3.1.3. Lemma. Let $\mathcal{A}$ be the category of $T$-algebras in $\mathcal{C}$. Defined as above, $\bigcirc$ is a bifunctor on $\mathcal{A}$. It satisfies associativity conditions together with coherence. The associativity isomorphisms are preserved by the forgetful functor $\mathfrak{U}: \mathcal{A} \rightarrow \mathcal{C}$.

Proof. To show that $\bigcirc$ is a bifunctor we have to show first that its value on a pair of objects in $\mathcal{A}$ is again in $\mathcal{A}$, i.e. that the following diagram is commutative

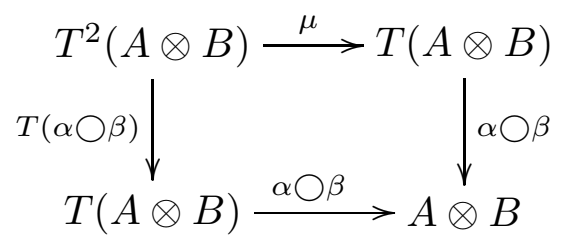


This follows from the condition a) in the Definition 3.1.1 and the commutativity of the following diagram:

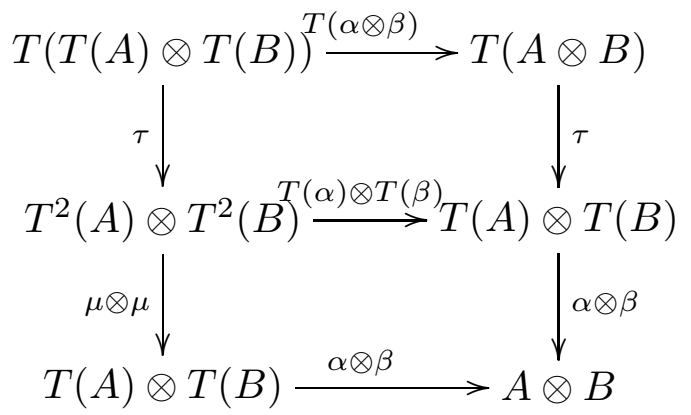

The upper square of this diagram is commutative because $T$ and $\otimes$ are functors and $\tau$ is a natural transformation. The lower square is commutative because it is the result of an application of $\otimes$ to two commutative squares (representing the fact that $\alpha$ and $\beta$ are structure morphisms for $T$-algebras).

Thus on objects $\bigcirc$ behaves like a bifunctor on $\mathcal{A}$. We have to show that $f \bigcirc g$ is a morphism in $\mathcal{A}$ for any two morphisms $f:\left(A_{1}, \alpha_{1}\right) \rightarrow\left(A_{2}, \alpha_{2}\right)$ and $g:\left(B_{1}, \beta_{1}\right) \rightarrow$ $\left(B_{2}, \beta_{2}\right)$ in $\mathcal{A}$, i.e. the following diagram is commutative

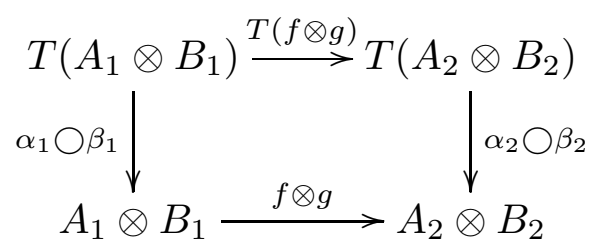

By the definition of $\bigcirc$, this diagram can be decomposed as follows

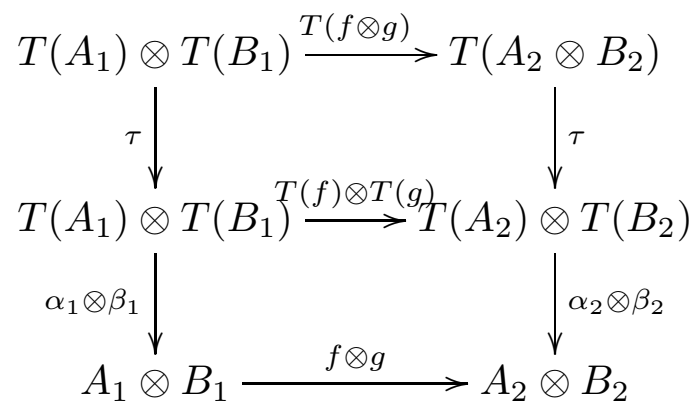

The upper square of this diagram is commutative because $\otimes$ and $T$ are functors and $\tau$ is a natural transformation. The lower square is commutative because it is 
the result of an application of $\otimes$ to two commutative squares (expressing the fact that $f$ and $g$ are morphisms of $T$-algebras).

Clearly pairs of identities are mapped to identities by $\bigcirc$ and the composition is preserved, i.e. $\bigcirc$ is a bifunctor on $\mathcal{A}$.

It remains to show that $\bigcirc$ is coherently associative and that the forgetful functor to $\mathcal{C}$ preserves the associativity isomorphisms. The former claim actually follows from the latter, since $\otimes$ is coherently associative. So all we have to do is to show that the $\otimes$-associativity isomorphisms between the images of the forgetful functor belong to $\mathcal{A}$, i.e. the outer rectangle of the following diagram is commutative for all $A_{1}, A_{2}, A_{3} \in \mathcal{A}$

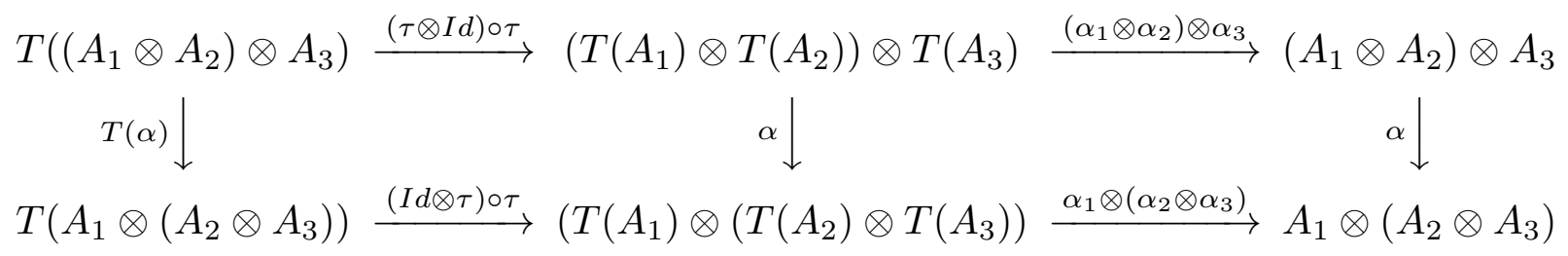

The right hand square of this picture is commutative because $\otimes$ and $T$ are functors and $\alpha$ is a natural transformation. Commutativity of the left hand square is the contents of condition b) of Definition 3.1.1.

Having extended the product $\otimes$ from $\mathcal{C}$ to $\mathcal{A}$, we would like to know if this extension possesses a left adjoint, given $\otimes$ does so on $\mathcal{C}$. We can infer it easily from the adjoint lifting theorem, if we assume that $\mathcal{A}$ has all coequalizers. For the question of when a category of algebras over a triple has all coequalizers see e.g. [BarW], Section 9.3.

3.1.4. Proposition. Suppose that $\mathcal{A}$ has all coequalizers and for every $A \in \mathcal{A}$ the functor $\mathfrak{U}(A) \otimes-: \mathcal{C} \rightarrow \mathcal{C}$ has a left adjoint. Then the functor $A \bigcirc-: \mathcal{A} \rightarrow \mathcal{A}$ has a left adjoint as well.

Proof. It is clear that $\mathfrak{U}$ is a monadic functor ([Bo1], Definition 4.4.1) and by our construction $\mathfrak{U}(A \bigcirc-)=\mathfrak{U}(A) \otimes \mathfrak{U}(-)$, therefore (e.g. [Bo2], Theorem 4.5.6) since $\mathfrak{U}(A) \otimes-$ has a left adjoint, so does $A \bigcirc-$. This shows our assertion.

Here we define $\underline{\operatorname{cohom}}(A, B)$ is an object that represents $\operatorname{Hom}(A, B \bigcirc-)$. From the last proposition we know that on $\mathcal{A}$ there is a $\underline{\operatorname{cohom}}(-,-)$ that is functorial in the first argument. Since Yoneda embedding is full and faithful, we conclude that our construction is actually a bifunctor.

3.2. Derived $\underline{\text { cohom }}$. By now we have constructed $\underline{\text { cohom }}(-, A)$ for an algebra $A \in \mathcal{A}$, given existence of $\underline{\text { cohom }}$ on $\mathcal{C}$. Now assume that $\mathcal{C}$ carries in addition a 
closed model structure, such that $C \otimes-$ is a right Quillen functor for any $C \in \mathcal{C}$, i.e. it has a left adjoint and it maps fibrations and trivial fibrations to the like. Then, by a general result, $\underline{\operatorname{cohom}}(-, C)$ is a left Quillen functor and we can define its left derived version.

Suppose that we can transport closed model structure from $\mathcal{C}$ to $\mathcal{A}$ through the adjunction of the free algebra and the forgetful functor, i.e. we can introduce a closed model structure on $\mathcal{A}$ so that a map in $\mathcal{A}$ is a weak equivalence or fibration if and only its image under the forgetful functor is such. Then for any $A \in \mathcal{A}$ we have that $A \bigcirc-$ is a right Quillen functor on $\mathcal{A}$, and hence $\underline{\operatorname{cohom}}(-, A)$ is a left Quillen functor and we can define derived $\underline{\text { cohom }}$ as follows:

$$
\mathbb{L} \underline{\operatorname{cohom}}(-, A):=\underline{\operatorname{cohom}}(L(-), A)
$$

where $L$ is a cofibrant replacement functor on $\mathcal{A}$.

It remains only to analyze when such a transport of model structure is possible. The general situation of a transport was considered in several papers e.g. [Bek] [Bl], [CaGa], [Cra], [Q], [R], [S]. For our purposes it is enough to consider locally presentable categories with cofibrantly generated model structures. In this case the conditions on the forgetful functor are quite mild.

Moreover, we are mostly interested in the particular case of operads in the category of algebras over a Hopf operad in the category of dg complexes of vector spaces over a field. In the next Section we will show that categories of such operads can be constructed as categories of algebras over certain triples on the category of $\mathrm{dg}$ complexes of vector spaces. Then the machinery of transport of model structure can be applied to these triples directly.

\section{$\S 4$. Iteration of algebraic constructions}

4.0. Introduction. As it is described in Section 3, if a triple is Hopf-like with respect to a monoidal structure, it is easy to extend cohom from the ground category to the category of algebras over this triple. Hopf operads provide examples of such triples, given that monoidal structure on the ground category is distributive with respect to the direct sum. However, this is not always the case.

Consider the category $\mathfrak{A}$ of associative algebras in the category of vector spaces over a field. Since the operad of associative algebras is Hopf, $\mathfrak{A}$ has a symmetric monoidal structure, given by the tensor product of algebras. On $\mathfrak{A}$ this monoidal structure is not distributive with respect to direct sums, that is, free products of algebras. In fact, given $A_{1}, A_{2}, A_{2} \in \mathfrak{A}$, in general we have no isomorphism between 
$A_{1} \otimes\left(A_{2} \coprod A_{3}\right)$ and $\left(A_{1} \otimes A_{2}\right) \coprod\left(A_{1} \otimes A_{3}\right)$. Thus we cannot represent operads in $\mathfrak{A}$ as algebras over a triple, and constructions of Section 3 concerning existence of $\underline{\text { cohom }}$ do not apply to the category of operads in $\mathfrak{A}$.

However, we can overcome these difficulties by working with the operads instead of their categories of algebras. In the example above we can represent operads in the category of associative algebras as algebras over a colored operad in the category of vector spaces.

For example, a classical operad in $\mathfrak{A}$ is a sequence $A:=\left\{A_{n}\right\}_{n \in \mathbb{Z}_{>0}}$ of objects of $\mathfrak{A}$ and morphisms

$$
\gamma_{m_{1}, \ldots, m_{n}}: A_{n} \otimes A_{m_{1}} \otimes \ldots \otimes A_{m_{n}} \rightarrow A_{m_{1}+\cdots+m_{n}}
$$

satisfying the usual axioms. The additional structures of associative algebras on $A_{n}$ 's are described by a sequence of morphisms of vector spaces: $\alpha_{n}: A_{n} \otimes A_{n} \rightarrow$ $A_{n}$, that satisfy the usual associativity axioms.

Compatibility of the operadic structure morphisms with the structures of associative algebras on individual $A_{n}$ 's is expressed by commutativity of the following diagrams:

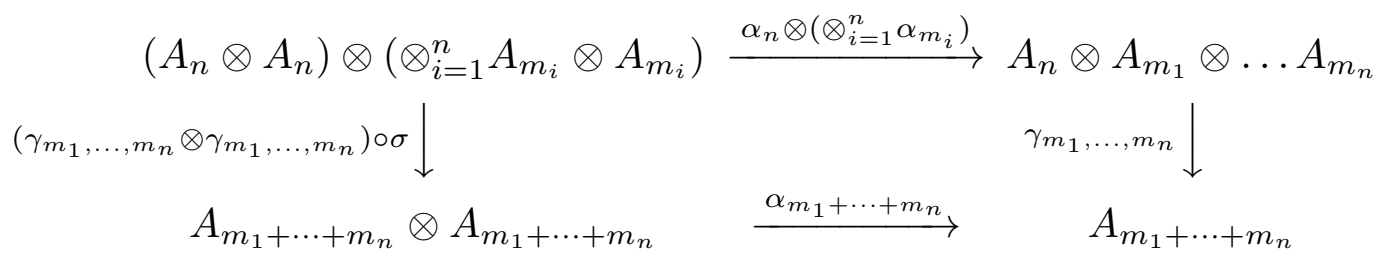

where $\sigma$ is the appropriate rearrangement of factors in the tensor products of vector spaces.

It is easy to see that we can express all of these conditions on the sequence $\left\{A_{n}\right\}$ as an action of a colored symmetric operad. Thus the category of classical operads in $\mathfrak{A}$ is equivalent to a category of algebras over a colored operad in vector spaces. Therefore we have the free algebra construction, results of Section 3 apply, and $\underline{\text { cohom }}$ extends to classical operads in $\mathfrak{A}$.

We would like to do the same in the case of general operads, as described in Section 1. To achieve the necessary degree of generality we will work with triples, which will include all operadic cases, given that the ground category has monoidal structure, which is distributive with respect to the direct sums.

The usual definition of a triple on a category $\mathcal{C}$ consists of three parts: a functor $T: \mathcal{C} \rightarrow \mathcal{C}$, and natural transformations $\zeta: T \circ T \rightarrow T, \eta: I d \rightarrow T$, satisfying 
certain associativity and unit axioms. The functor $T$ is supposed to represent the "free algebra" construction, while $\zeta$ and $\eta$ represent composition and the identity operation respectively.

Often we have more information about the triple than contained in its definition as above. We have a grading on the "free algebra" construction, given by the arity of operations involved, i.e. we can decompose $T(C)(C \in \mathcal{C})$ into a direct sum of $T_{n}(C)\left(n \in \mathbb{Z}_{>0}\right)$, where $T_{n}(C)$ stands for applying to "generators" $C$ all of the " $n$-ary operations" $T_{n}$.

Such decomposition of $T$ is very helpful, since usually $T_{n}$ 's behave better with respect to monoidal structure and other triples than the whole $T$ : see [Va3] for a closely related discussion. Using this we can mimic construction of a colored operad, as in the case of classical operads in associative algebras above, in the more general situation of decomposed triples.

In order to do so we have to formalize the notion of triples admitting such a decomposition. We should axiomatize the relationship between $T_{n}$ 's for different $n$ 's, so that the combined object would satisfy the associativity and the unit axioms, stated in the usual definition of a triple. The best way to do so is via representations of operads in categories.

It is often the case (in particular it is so for operads as described in Section 1 ) that the " $n$-ary operations" $T_{n}$ is not just a functor on $\mathcal{C}$, but is given as a composition

$$
\mathcal{C} \stackrel{\Delta}{\rightarrow} \mathcal{C}^{\times^{n}} \stackrel{G_{n}}{\rightarrow} \mathcal{C}
$$

where $\Delta$ is the diagonal functor and $G_{n}$ is some functor $\mathcal{C}^{\times^{n}} \rightarrow \mathcal{C}$. In such cases it is convenient to work with representations of operads on $\mathcal{C}$, i.e. with morphisms of operads with codomain $\left\{F u n\left(\mathcal{C}^{\times^{n}}, \mathcal{C}\right)\right\}_{n \in \mathbb{Z}_{n>0}}$.

So we will consider strict operads in the category of categories and work with lax morphisms of such operads (i.e. with morphisms that commute with the structure functors only up to a natural transformation). A lax morphism into an endomorphism operad of a category will give us a generalized triple that under some conditions can be transformed into a usual triple. In such cases we will say that the triple is operad-like. The technique of lax morphisms was invented long ago (cf. $[\mathrm{KS}]$ ) and applied recently to the case of operads in [Bat].

We proceed as follows: we start with the well known notion of strict pseudooperads in categories, we organize them in a category and then extend it to include lax morphism of operads, which satisfy natural conditions of coherence (later these conditions will be shown to correspond to the associativity axioms of triples). Then 
we consider the notion of a strict operad in categories (i.e. with a unit) and define lax morphisms of such objects. Again we will need some coherence conditions, which later will turn out to be the unit axioms of a triple. Finally we define operad-like triples as lax morphisms into the endomorphism operad of a category and we finish by proving that iteration of algebraic constructions preserves existence of cohom .

4.1. Notation. We will denote functors usually by capital letters (both Greek and Latin), whereas natural transformations will be denoted by small letters.

Commutative diagrams of functors will be rarely commutative on the nose, instead we will have to endow them with natural transformations, making them commutative. When we draw a diagram as follows

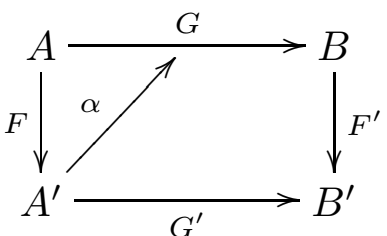

and say that $\alpha$ makes the diagram commutative, we mean that $\alpha$ is a natural transformation $G^{\prime} \circ F \rightarrow F^{\prime} \circ G$. Notice that $\alpha$ is not supposed to be an isomorphism of functors, any functor morphism is acceptable. Similarly for the diagram

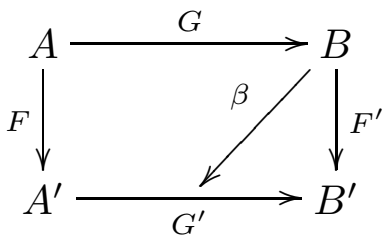

We will often encounter one of the following two situations:
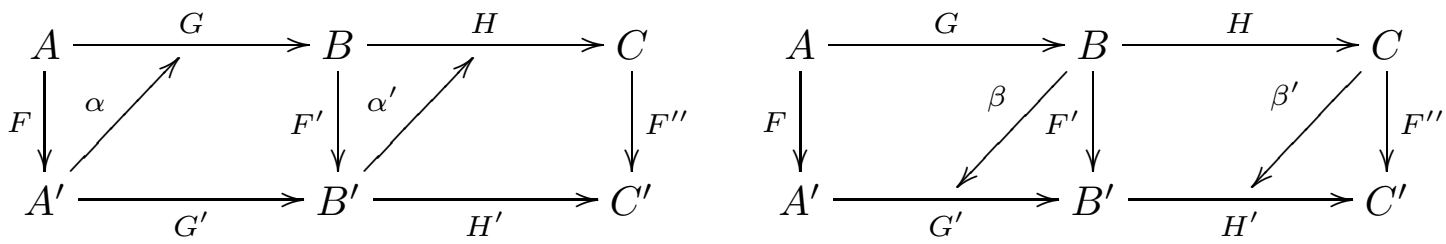

There is a well known procedure of pasting natural transformations in such cases. In order to fix notation, we will write the relevant formulas explicitly. 
4.1.1. Definition. These compositions are defined as follows

$$
\begin{aligned}
& \left(\alpha^{\prime} * \alpha\right)_{a}:=\alpha_{G(a)}^{\prime} \circ H^{\prime}\left(\alpha_{a}\right), \quad a \in A, \\
& \left(\beta * \beta^{\prime}\right)_{a}:=H^{\prime}\left(\beta_{a}\right) \circ \beta_{G(a)}^{\prime}, \quad a \in A .
\end{aligned}
$$

Direct computation easily shows that both compositions are indeed natural transformations $H^{\prime} \circ G^{\prime} \circ F \rightarrow F^{\prime \prime} \circ H \circ G$ and $F^{\prime \prime} \circ H \circ G \rightarrow H^{\prime} \circ G^{\prime} \circ F$ respectively. Moreover, the composition $*$ is associative.

4.2. Strict pseudo-operads in categories. Let $\mathcal{C} \mathcal{A T}$ be a small category whose objects are some small categories and morphisms are functors. We will assume that it is sufficiently rich so that all the following constructions make sense. In particular, we have a symmetric monoidal structure $\times$ given by the direct product and a choice of a category $E$ with one object and one morphism (identity). Thus we can consider pseudo-operads in $\mathcal{C} \mathcal{A} \mathcal{T}$.

Although our goal is to prove existence of $\underline{\text { cohom }}$ for operads, these being defined in the general way as in Section 1, the categorical operads that we will use will be solely the classical ones. The reason for this is that the categories of graphs themselves, that were used in Section 1 in definition of operads, are in fact examples of classical operads in categories: see Section 4.9 .3 below. Thus for our purposes there is no need to consider more general categorical operads, than the classical ones.

4.2.1. Definition. A strict pseudo-operad in categories is a classical nonsymmetric pseudo-operad in $(\mathcal{C} \mathcal{A} \mathcal{T}, \times)$ (e.g. [MarShSt] Definition 1.18). We will denote the category of strict pseudo-operads in $\mathcal{C} \mathcal{A} \mathcal{T}$ by $\Psi \mathcal{O P}{ }_{\text {st }}(\mathcal{C} \mathcal{A} T)$. The structure functors of an object of $\Psi \mathcal{O P} \mathcal{P}_{s t}(\mathcal{C A T})$ will be denoted by $\left\{\Upsilon_{m_{1}, . ., m_{n}}\right\}_{m_{i} \in \mathbb{Z}_{>0}}$.

We could have used of course the notion of a 2-pseudo-operad in categories and work in the more general setting of higher operads, but for our needs in this paper strict operads in categories will suffice. One of the reasons for this restriction is the following example.

4.2.2. Example. One of the most important examples of a strict pseudo-operad in categories is the endomorphism pseudo-operad $\mathfrak{E}(\mathcal{C})$ of a category $\mathcal{C}$. It is defined as follows:

$$
\mathfrak{E}(\mathcal{C})_{n}:=\operatorname{Fun}\left(\mathcal{C}^{\times^{n}}, \mathcal{C}\right), \quad n \in \mathbb{Z}_{>0},
$$

where Fun stands for the category of functors. The structure morphisms are given by compositions of functors. 
4.3. Lax morphisms between strict pseudo-operads. As we have noted above we could have worked with 2-operads in categories instead of the usual ones, i.e. we could have incorporated natural transformations in operadic structure. However, as the example of the endomorphism operad of a category shows, it is enough for many purposes, and in particular for ours, to consider only classical operads. Yet when we start organizing these operads in categories we have to take into account the natural transformations, that we have omitted before.

We have already the category $\Psi \mathcal{O P}{ }_{s t}(\mathcal{C} \mathcal{A} \mathcal{T})$, whose morphisms are sequences of functors, that commute on the nose with the structure functors of pseudo-operads. This rarely happens. In most cases we have a natural transformation making these diagrams commutative. Later we will see that it is these natural transformations that define the multiplication for the triples that we will consider. So we need to enlarge $\Psi \mathcal{O P}{ }_{s t}(\mathcal{C} \mathcal{A} \mathcal{T})$ to include not only strict but also lax morphisms of operads. Lax morphisms were invented a long time ago (see e.g. [KS] Section 3). For completeness we reproduce explicit definitions here. Our treatment will deviate from the classical one only when we will consider relative version of the construction (i.e. categories of lax morphisms with constant codomain) and more importantly when we introduce the notion of an equivariant operad in categories. Both of these constructions are specifically tailored for treatment of triples.

4.3.1. Definition. Let $P, P^{\prime}$ be two strict pseudo-operads in categories. $A$ lax morphism from $P$ to $P^{\prime}$ is a sequence of functors $\left\{F_{n}: P_{n} \rightarrow P_{n}^{\prime}\right\}_{n \in \mathbb{Z}_{>0}}$ and a sequence of natural transformations $\left\{\zeta_{m_{1}, . ., m_{n}}\right\}$, making the following diagram commutative:

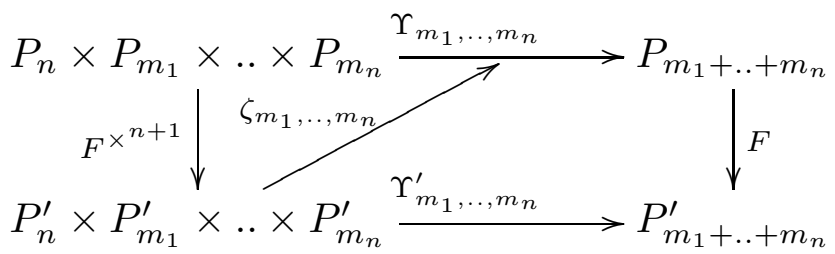

Given two lax morphisms $\left(\left\{F_{n}\right\},\left\{\zeta_{m_{1}, . ., m_{n}}\right\}\right): P \rightarrow P^{\prime}$ and $\left(\left\{F_{n}^{\prime}\right\},\left\{\zeta_{m_{1}, . ., m_{n}}^{\prime}\right\}\right):$ $P^{\prime} \rightarrow P^{\prime \prime}$ we define their composition to be $\left(\left\{F_{n}^{\prime} \circ F_{n}\right\},\left\{\zeta_{m_{1}, . ., m_{n}} * \zeta_{m_{1}, . ., m_{n}}^{\prime}\right\}\right)$, where * is composition of natural transformations, as defined in 4.1.1.

It is easy to see that pseudo-operads in categories and lax morphisms form a category. From the the pasting theorem of [Pow], we know that composition of $\zeta$ 's is associative and therefore composition of the whole morphisms is associative. There is an identity lax morphism for every category, given by the identity functor and the trivial natural automorphism of it. 
However, we are interested in a subcategory of this category, consisting of lax morphisms, that have the property of coherence. As usual coherence means that different ways of composing natural transformations are equal. Later we will see that these conditions will translate into associativity properties of the multiplication natural transformations of triples that we will construct.

4.3.2. Definition. Let $P$ and $P^{\prime}$ be two strict pseudo-operads in categories. Let $(F, \zeta): P \rightarrow P^{\prime}$ be a lax morphism between them. We say that $(F, \zeta)$ is coherent if the *-compositions of natural transformations in the following two diagrams are equal:
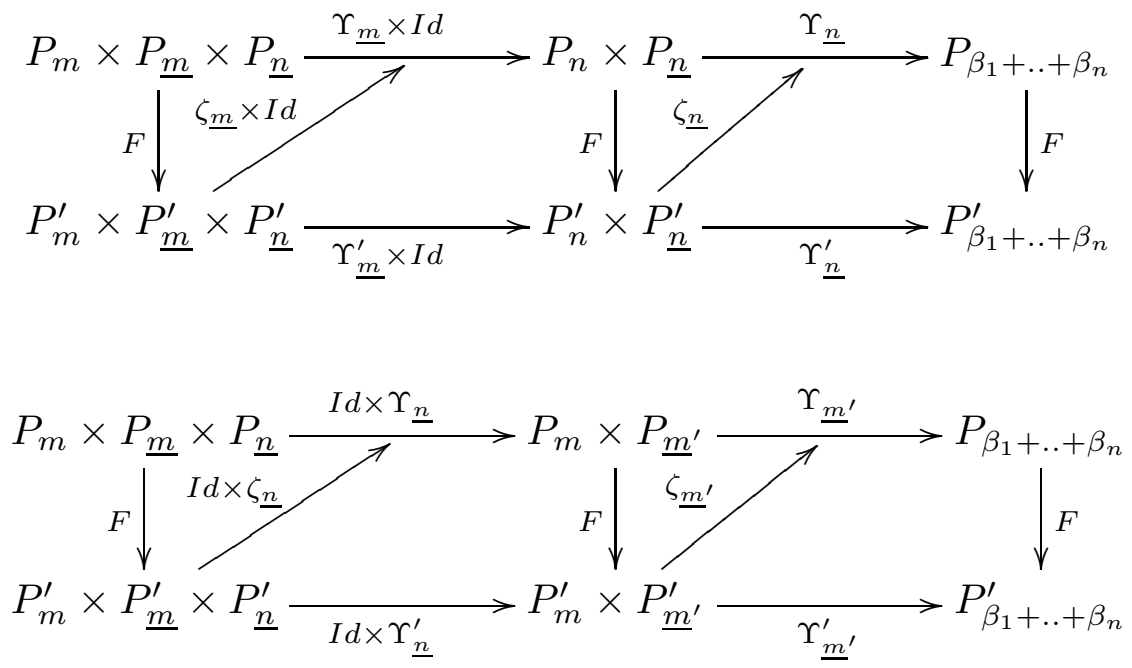

where $\underline{m}:=\left\{\alpha_{1}, . ., \alpha_{m}\right\}, \underline{n}:=\left\{\beta_{1}, . ., \beta_{n}\right\}, \underline{m}^{\prime}:=\left\{\beta_{1}+. .+\beta_{\alpha_{1}}, . ., \beta_{\alpha_{1}+. .+\alpha_{m-1}+1}+\right.$ ..$\left.+\beta_{n}\right\}, \alpha_{1}+. .+\alpha_{m}=n, \alpha_{i}, \beta_{i} \in \mathbb{Z}_{>0}$, and $P_{\underline{m}}:=P_{\alpha_{1}} \times \ldots \times P_{\alpha_{m}}$, similarly for $P_{\underline{n}}, P_{\underline{m}^{\prime}}$ and $P^{\prime}$.

4.3.3. Proposition. Strict pseudo-operads in categories and coherent lax morphisms form a subcategory of the category of strict pseudo-operads and lax morphisms

Proof. It is clear that the identity lax morphism for any strict pseudo-operad is coherent. We have to prove that composition of coherent lax morphisms is again coherent.

Let $P \stackrel{(F, \zeta)}{\rightarrow} P^{\prime} \stackrel{\left(F^{\prime}, \zeta^{\prime}\right)}{\rightarrow} P^{\prime \prime}$ be a sequence of coherent lax morphisms between strict pseudo-operads in categories. We have to show that the *-product of $\zeta$ and $\zeta^{\prime}$ provides a unique way of making diagrams commutative, i.e. compositions of the natural transformations as in diagrams (4.1) are equal. But these diagrams are the 
outer rectangles of the following diagrams
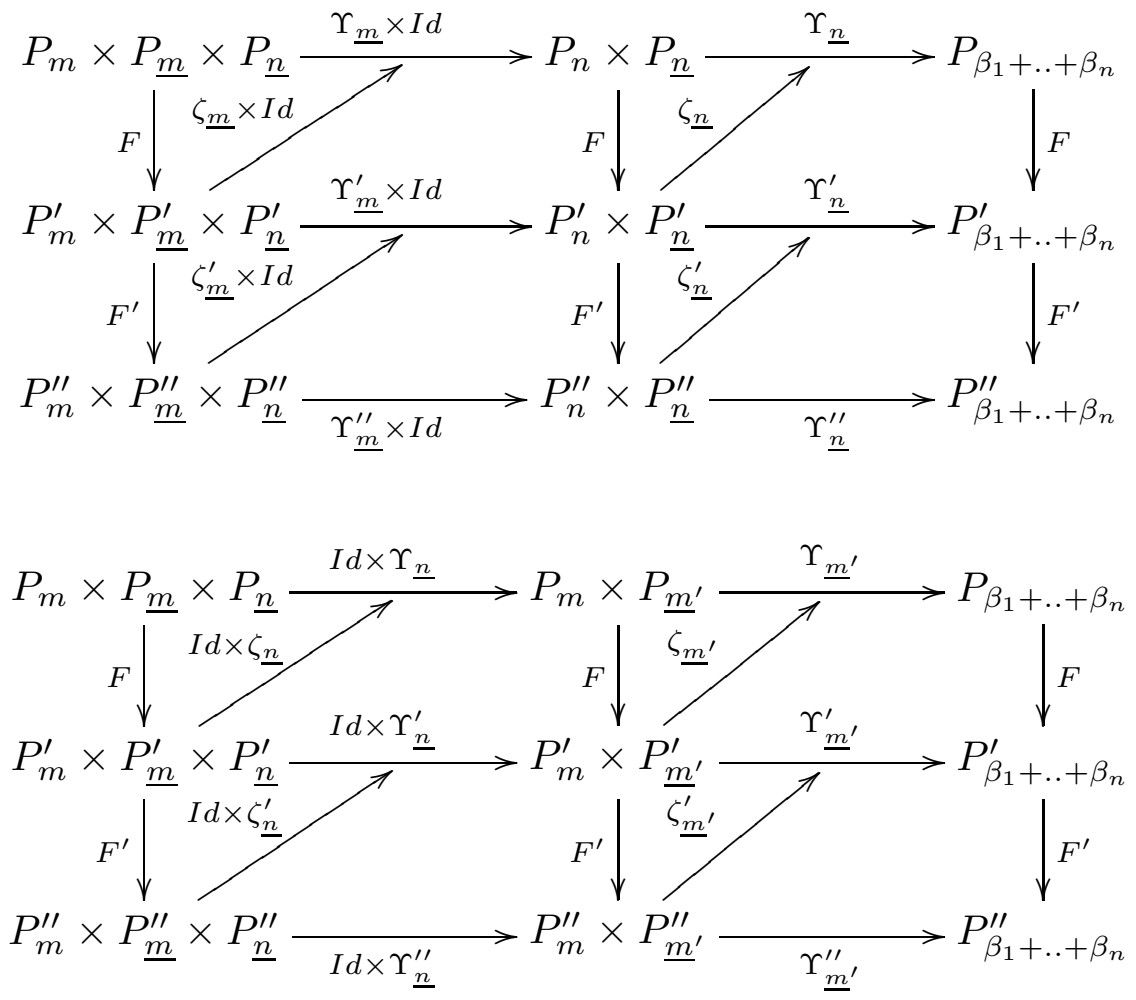

Since $(F, \zeta)$ is coherent, compositions of natural transformations in the first rows of these diagrams are equal, similarly for the second rows. Therefore compositions of first the rows and then the columns are equal. We would like to show that compositions of first the columns and then the rows are equal as well.

This follows from the pasting theorem in [Pow].

4.3.4. Notation. We will denote the category of strict pseudo-operads in categories and coherent lax morphisms by $\Psi \mathcal{O P}(\mathcal{C A T})$.

4.4. Categories of pseudo-operads over a pseudo-operad. As with any category, it is necessary sometimes to consider an object $P$ in $\Psi \mathcal{O P}(\mathcal{C A T})$ and all morphisms in $\Psi \mathcal{O P}(\mathcal{C} \mathcal{A T})$ with codomain $P$. It will be very important for us when we will work with representations of categorical operads on a category, i.e. when $P$ is the endomorphism operad for some category $\mathcal{C}$.

We would like of course to organize all these morphisms into a category - the category of pseudo-operads over $P$. But first we have to decide what shall we call a 
morphism between two such morphisms. In the standard way (i.e. without presence of 2-morphisms) we would define a morphism to a be a commutative (on the nose) triangle as follows

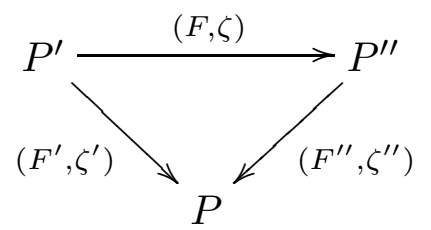

However, we have natural transformations and we should take them into account, i.e. we should define a morphism to be a diagram with a natural transformation making it commutative

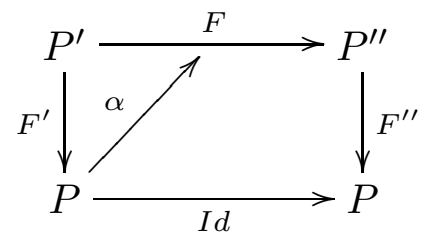

Now we have to decide how $\zeta, \zeta^{\prime}, \zeta^{\prime \prime}$ and $\alpha$ should relate to each other. If we did not have $\alpha$, then the relation would have been $\zeta^{\prime}=\zeta * \zeta^{\prime \prime}$. Having $\alpha$ we can put all of these natural transformation into one big diagram

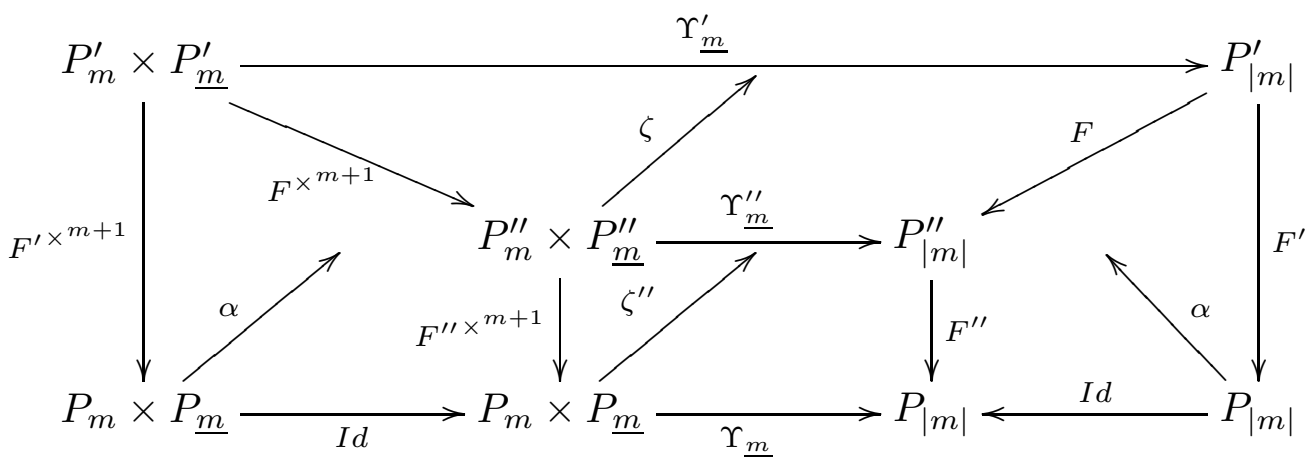

where for typographical reasons we have omitted $\zeta^{\prime}$, that makes the outer rectangle commutative.

We see that there are two ways to construct natural transformations

$$
\Upsilon_{\underline{m}} \circ F^{\prime \times^{m+1}} \rightarrow F^{\prime \prime} \circ F \circ \Upsilon_{\underline{m}}^{\prime} .
$$

One is $\zeta * \zeta^{\prime \prime} * \alpha$ and the other is $\alpha * \zeta^{\prime}$. Their equality is the natural condition of compatibility. 
4.4.1. Definition. The category $\Psi \mathcal{O P}(\mathcal{C A T}) / / P$ has coherent lax morphisms with codomain $P$ as objects, and for any two such morphisms $\left(F^{\prime}, \zeta^{\prime}\right): P^{\prime} \rightarrow P$, $\left(F^{\prime \prime}, \zeta^{\prime \prime}\right): P^{\prime \prime} \rightarrow P$, a morphism from the first one to the second is a pair $((F, \zeta), \alpha)$, where $(F, \zeta): P^{\prime} \rightarrow P^{\prime \prime}$ is a coherent lax morphism of strict pseudo-operads, and $\alpha$ is a natural transformation, making diagram (4.2) commutative and satisfying

$$
\zeta * \zeta^{\prime \prime} * \alpha=\alpha * \zeta^{\prime}
$$

4.4.2. Proposition. Constructed as above $\Psi \mathcal{O P}(\mathcal{C A T}) / / P$ is indeed a category.

Proof. A morphism in $\Psi \mathcal{O P}(\mathcal{C} \mathcal{A} \mathcal{T}) / / P$ consists of a coherent lax morphism and a natural transformation. From Definition 4.1.1 and Proposition 4.3.3 we know how to compose both types, so the composition in $\Psi \mathcal{O P}(\mathcal{C} \mathcal{A T}) / / P$ is clear.

Since by the pasting theorem composition of natural transformations is associative (Lemma 4.1.2) and we know that this is true for coherent lax morphisms (Proposition 4.3.3), all we have to do now is to show that the condition, formulated in Definition 4.4.1 is satisfied by the composition.

So let $\left(F^{\prime}, \zeta^{\prime}\right): P^{\prime} \rightarrow P,\left(F^{\prime \prime}, \zeta^{\prime \prime}\right): P^{\prime \prime} \rightarrow P$ and $\left(F^{\prime \prime \prime}, \zeta^{\prime \prime \prime}\right): P^{\prime \prime \prime} \rightarrow P$ be three coherent lax morphisms, with codomain $P$. Suppose we have two morphisms $((G, \psi), \alpha):\left(F^{\prime}, \zeta^{\prime}\right) \rightarrow\left(F^{\prime \prime}, \zeta^{\prime \prime}\right),\left(\left(G^{\prime}, \psi^{\prime}\right), \alpha^{\prime}\right):\left(F^{\prime \prime}, \zeta^{\prime \prime}\right) \rightarrow\left(F^{\prime \prime \prime}, \zeta^{\prime \prime \prime}\right)$, that satisfy conditions of Definition 4.4.1. We can organize everything into one diagram as follows

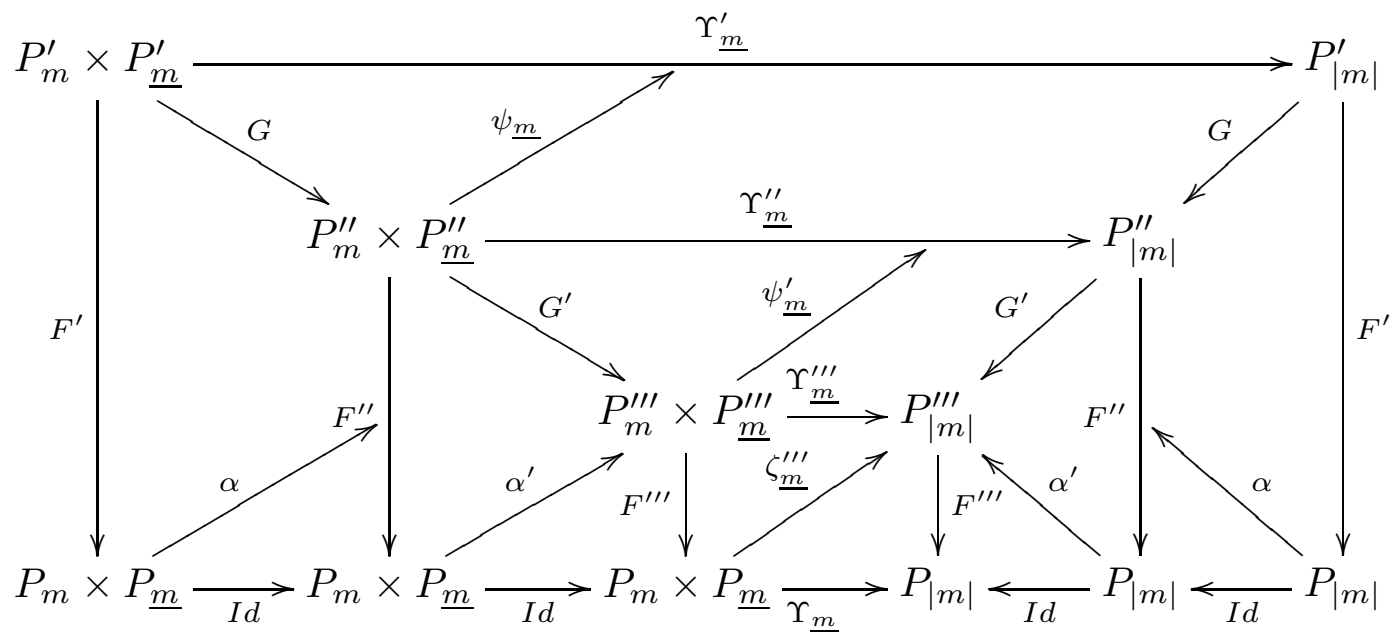


Since $((G, \psi), \alpha)$ and $\left(\left(G^{\prime}, \psi^{\prime}\right), \alpha^{\prime}\right)$ satisfy conditions of definition 4.4 .1 we have the following equalities

$$
\psi^{\prime} * \zeta^{\prime \prime \prime} * \alpha^{\prime}=\alpha^{\prime} * \zeta^{\prime \prime}, \quad \psi * \zeta^{\prime \prime} * \alpha=\alpha * \zeta^{\prime} .
$$

Using these equalities we get the following

$$
\left(\alpha^{\prime} * \alpha\right) * \zeta^{\prime}=\alpha^{\prime} * \psi * \zeta^{\prime \prime} * \alpha=\psi * \alpha^{\prime} * \zeta^{\prime \prime} * \alpha=\left(\psi * \psi^{\prime}\right) * \zeta^{\prime \prime \prime} *\left(\alpha^{\prime} * \alpha\right),
$$

where the second equality is justified by the pasting theorem ([Pow $]$ ). The combined equality is exactly the condition as in the Definition 4.4 .1 for the composition.

Later we will often work with representations of categorical operads on a category, i.e. we will study lax morphisms into the endomorphism operad. We will want to construct a functor from the category of certain representations to the category of triples on that category. For that we will need a notion of the category of representations. One candidate is obviously the category of operads over the endomorphism operad, as constructed above. However, it will prove to be too relaxed. We will need a somewhat more restricted notion. Namely we will consider the subcategory with the same objects but only strict morphisms.

4.4.3. Notation. We will denote by $\Psi \mathcal{O P}{ }_{s t}(\mathcal{C} \mathcal{A T}) / / P$ the subcategory of $\Psi \mathcal{O P}(\mathcal{C} \mathcal{A} \mathcal{T}) / / P$, consisting of the same objects as $\Psi \mathcal{O P}(\mathcal{C} \mathcal{A} \mathcal{T}) / / P$, but for any pair of them $\left(P^{\prime}, F^{\prime}, \zeta^{\prime}\right)$ and $\left(P^{\prime \prime}, F^{\prime \prime}, \zeta^{\prime \prime}\right)$, a morphism $((F, \zeta), \alpha)$ from the first to the second is in $\Psi \mathcal{O P} \mathcal{P}_{s t}(\mathcal{C A T}) / / P$ if $(F, \zeta)$ is strict, i.e. $\zeta$ is the identity.

Since strict morphisms form a subcategory of the category of lax morphisms, we see that $\Psi \mathcal{O P} \mathcal{P}_{s t}(\mathcal{C A T}) / / P$ is indeed a subcategory of $\Psi \mathcal{O P}(\mathcal{C} \mathcal{A T}) / / P$.

4.5. Strict operads in categories. Until now we have considered pseudooperads. Now we would like to discuss also the unital version of our constructions. Since the category $\mathcal{C} \mathcal{A} \mathcal{T}$ is monoidal with a unit, we have the natural notion of an operad in $\mathcal{C} \mathcal{A} \mathcal{T}$, as before we restrict our attention only to the classical operads. Recall that $E$ is a choice of a category with one object and one morphism (identity).

4.5.1. Definition. A strict operad in categories is a classical non-symmetric operad (e.g. [MarShSt] Section 1.2) in the monoidal unital category $(\mathcal{C} \mathcal{A T}, \times, E)$. We will denote the category of strict operads in categories by $\mathcal{O P}_{\text {st }}(\mathcal{C A T})$.

As with pseudo-operads, we would like to extend the category $\mathcal{O P}_{s t}(\mathcal{C A T})$ to include lax morphism of operads. As usual that should mean making all diagrams, that before were commutative on the nose, commutative only up to natural transformations. First we list the relevant diagrams from the classical definition of operads. 
As defined above, a strict operad in categories is a pseudo-operad $P$ with a strict morphism of strict pseudo-operads $U: E \rightarrow P$, where we consider $E$ as a pseudooperad with $E_{n}:=\varnothing$ for $n>1$, and the obvious structure morphism. The strict morphism $U$ should make the following diagrams commutative
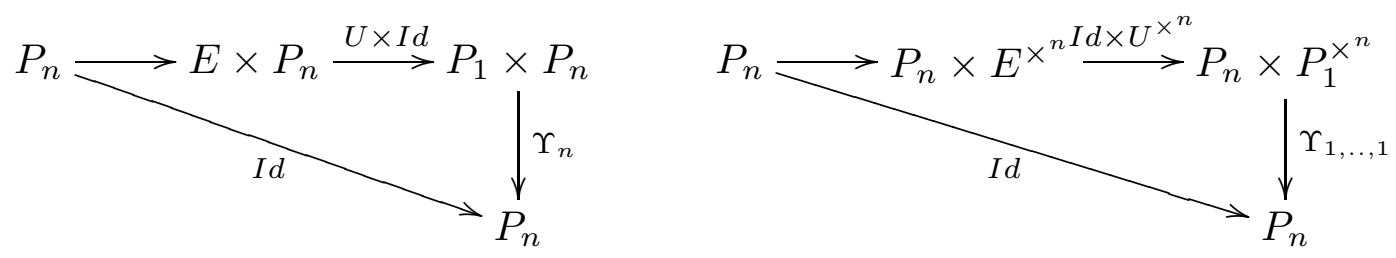

A strict morphism between strict operads commutes with these U's on the nose, i.e. we have the following commutative diagram for a strict morphism of operads $F: P \rightarrow P^{\prime}$

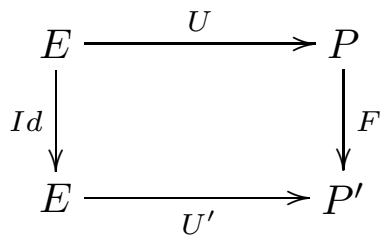

We would like our lax morphisms to do that only up to a natural transformation, that satisfies some coherence conditions. Later we will see that these conditions are translated into the usual unit axioms for triples.

4.5.2. Definition. Let $U: E \rightarrow P$ and $U^{\prime}: E \rightarrow P^{\prime}$ be two strict operads in categories. A coherent lax morphism from $P$ to $P^{\prime}$ is a coherent lax morphism of pseudo-operads $(F, \zeta): P \rightarrow P^{\prime}$ and a natural transformation $\eta$, making the following diagram commutative

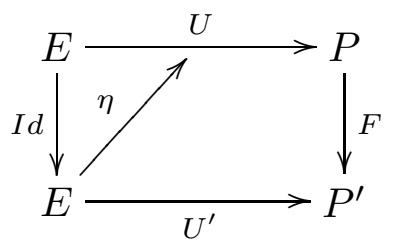

such that compositions of natural transformations in the following diagrams are identities

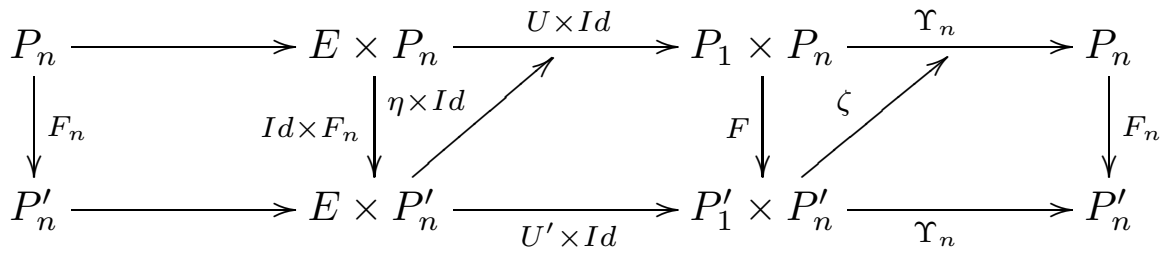




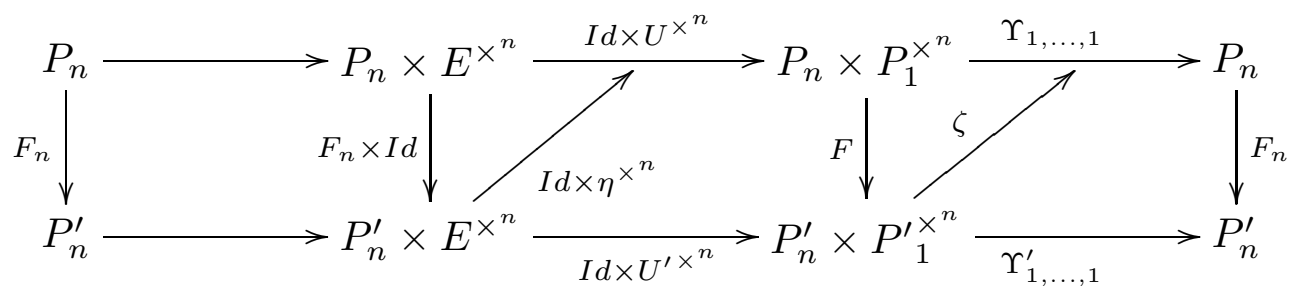

4.5.3. Proposition. Strict operads in categories and lax morphisms between them constitute a category

Proof. Let $(F, \zeta, \eta): P \rightarrow P^{\prime}$ and $\left(F^{\prime}, \zeta^{\prime}, \eta^{\prime}\right): P^{\prime} \rightarrow P^{\prime \prime}$ be two lax morphisms between strict operads in categories. We define their composition as $\left(F^{\prime} \circ\right.$ $\left.F, \zeta * \zeta^{\prime}, \eta * \eta^{\prime}\right)$, where $*$ denotes composition of natural transformations as defined in 4.1.1.

It is clear that for any strict operad, sequence of identity functors and identity natural transformations in place of $\zeta$ and $\eta$ constitute a lax morphism, and this morphism satisfies the conditions of identity with respect to the composition above. It remains to show that composition of lax morphisms is again a lax morphism and that this composition is associative.

To prove that composition is well defined we have to show that composition of natural transformations in the following diagrams are identities:

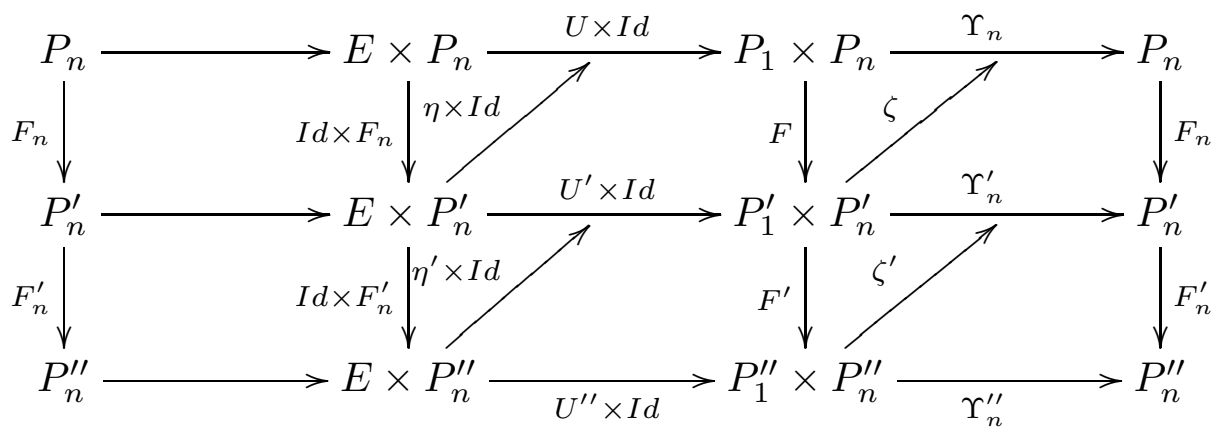




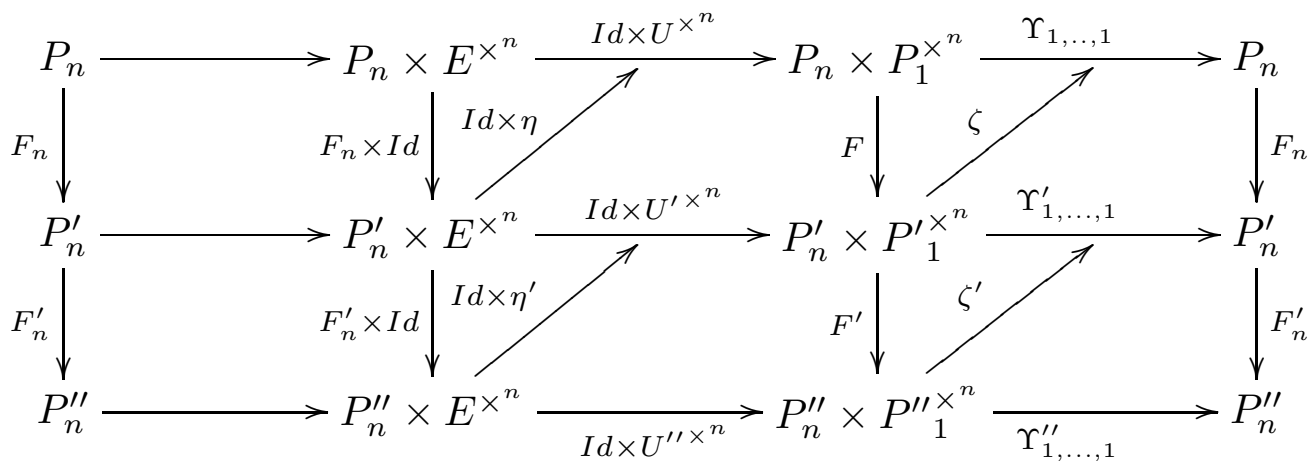

Arguing as in the proof of Proposition 4.3.3 we see that indeed compositions of these natural transformations are identities and hence composition of lax morphisms between strict operads in categories is well defined.

Since the composition of natural transformations is associative, in particular this is true for $\eta$ 's, hence strict operads in categories and lax morphisms between them indeed form a category.

4.5.4. Notation. We will denote the category of strict operads in categories and lax morphisms between them by $\mathcal{O P}(\mathcal{C A T})$.

4.6. Categories of operads over an operad. Since the endomorphism pseudo-operad of a category is obviously an operad, we would like to have a notion of a category of operads over an operad, similarly to the case of pseudo-operads, that we have considered in 4.4 .

Let $U: E \rightarrow P$ be a strict operad in categories. We want to organize morphisms in $\mathcal{O P}(\mathcal{C} \mathcal{A} \mathcal{T})$ with codomain $(P, U)$ into a category. Given two of them $\left(\left(F^{\prime}, \zeta^{\prime}\right), \eta^{\prime}\right)$ : $\left(P^{\prime}, U^{\prime}\right) \rightarrow(P, U)$ and $\left(\left(F^{\prime \prime}, \zeta^{\prime \prime}\right), \eta^{\prime \prime}\right):\left(P^{\prime \prime}, U^{\prime \prime}\right) \rightarrow(P, U)$ we would like to have the notion of a lax morphism from the first to the second. If we wanted only the ones coming from $\mathcal{O P}(\mathcal{C} \mathcal{A} \mathcal{T})$, we would have defined such a morphism as a lax morphism of operads (Definition 4.5.2) $((F, \zeta), \eta):\left(U^{\prime}, P^{\prime}\right) \rightarrow\left(P^{\prime \prime}, U^{\prime \prime}\right)$, such that 
the following diagram is commutative

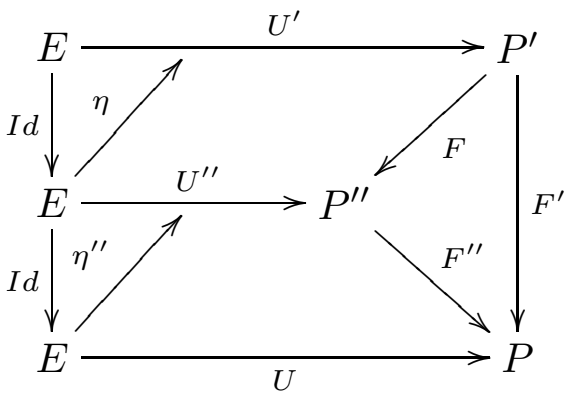

and we have the equality $\eta * \eta^{\prime \prime}=\eta^{\prime}$.

However, as in the case of pseudo-operads, we do not want an equality $F^{\prime \prime} \circ F=$ $F^{\prime}$, but we usually have a natural transformation $\alpha$, making the following diagram commutative

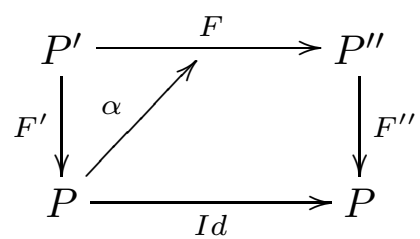

Obviously this natural transformation should satisfy the conditions of a morphism in $\Psi \mathcal{O P}(\mathcal{C} \mathcal{A} \mathcal{T}) / / P$, spelled out in Definition 4.4.1. In addition it should respect, in a sense, the unital structures $\eta, \eta^{\prime}$ and $\eta^{\prime \prime}$.

In the following diagram

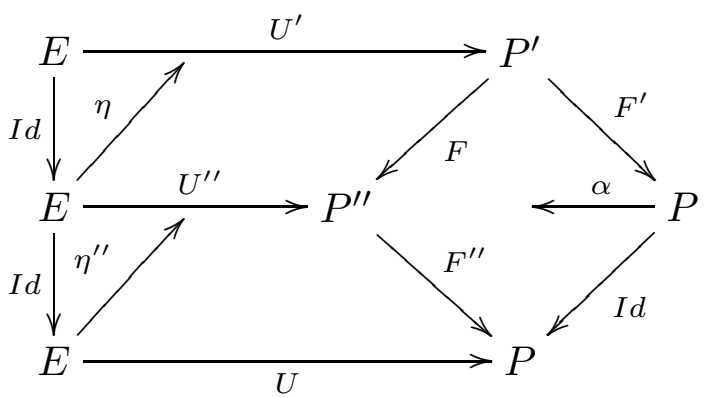

we see that there are two natural transformations $U \rightarrow F^{\prime \prime} \circ F \circ U^{\prime}$. One is $\eta * \eta^{\prime \prime}$ and the other is $\alpha * \eta^{\prime}$. Their equality is the natural compatibility condition between $\alpha$ and the unital structures. 
4.6.1. Definition. We will denote by $\mathcal{O P}(\mathcal{C A T}) / / P$ the category whose objects are coherent lax morphisms of strict operads with codomain $(P, U)$. Given two such morphisms $\left(\left(F^{\prime}, \zeta^{\prime}\right), \eta^{\prime}\right):\left(P^{\prime}, U^{\prime}\right) \rightarrow(P, U)$ and $\left(\left(F^{\prime \prime}, \zeta^{\prime \prime}\right), \eta^{\prime \prime}\right):\left(P^{\prime \prime}, U^{\prime \prime}\right) \rightarrow(P, U)$ a morphism from the first one to the second is a morphism $((F, \zeta), \eta):\left(P^{\prime}, U^{\prime}\right) \rightarrow$ $\left(P^{\prime \prime}, U^{\prime \prime}\right)$ in $\mathcal{O P}(\mathcal{C A T})$ and a natural transformation $\alpha$, such that $((F, \zeta), \alpha)$ is morphism $F^{\prime} \rightarrow F^{\prime \prime}$ in $\Psi \mathcal{O P}(\mathcal{C A T}) / / P$, and in addition we have

$$
\alpha * \eta^{\prime}=\eta * \eta^{\prime \prime} .
$$

4.6.2. Proposition. Defined as above $\mathcal{O P}(\mathcal{C A T}) / / P$ is indeed a category

Proof. Composition of two morphisms is inherited from the category of pseudooperads over a pseudo-operad. Identities are obviously present. All we have to do is to show that the composition of two morphisms, that satisfy conditions of the above definition, also satisfies these conditions.

Let $\left(F^{\prime}, \zeta^{\prime}, \eta^{\prime}\right):\left(P^{\prime}, U^{\prime}\right) \rightarrow(P, U),\left(F^{\prime \prime}, \zeta^{\prime \prime}, \eta^{\prime \prime}\right):\left(P^{\prime \prime}, U^{\prime \prime}\right) \rightarrow(P, U),\left(F^{\prime \prime \prime}, \zeta^{\prime \prime \prime}, \eta^{\prime \prime \prime}\right):$ $\left(P^{\prime \prime \prime}, U^{\prime \prime \prime}\right) \rightarrow(P, U)$ be three objects of $\mathcal{O P}(\mathcal{C} \mathcal{A} \mathcal{T}) / / P$. Let $((G, \psi), \phi, \alpha)$ be a morphism from the first to the second, and let $\left(\left(G^{\prime}, \psi^{\prime}\right), \phi^{\prime}, \alpha^{\prime}\right)$ be a morphism from the second to the third. We can organize everything into one diagram

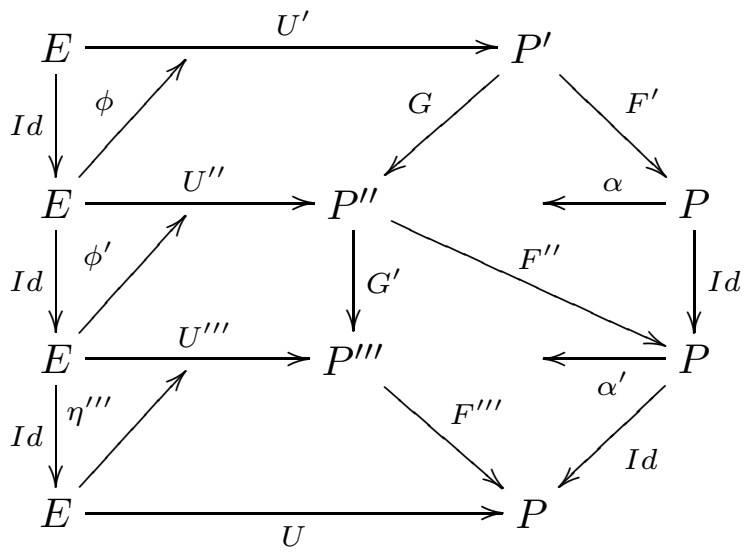

By assumption we have

$$
\alpha * \eta^{\prime}=\phi * \eta^{\prime \prime} \quad \alpha^{\prime} * \eta^{\prime \prime}=\phi^{\prime} * \eta^{\prime \prime \prime}
$$

Using these equalities we get a sequence

$$
\left(\alpha^{\prime} * \alpha\right) * \eta^{\prime}=\alpha^{\prime} * \phi * \eta^{\prime \prime}=\phi * \alpha^{\prime} * \eta^{\prime \prime}=\left(\phi * \phi^{\prime}\right) * \eta^{\prime \prime \prime},
$$


where in the second equality we have used the pasting theorem of [Pow]. The composite equality is exactly the condition, which $\alpha^{\prime} * \alpha$ should satisfy according to Definition 4.6.1.

As it was noted in 4.4, when we will consider representations of categorical operads on a category, we would like to consider lax morphisms into the endomorphism operad of that category, and we will want to organize these representations into a category, where as morphisms we take a strict subset of morphisms in $\mathcal{O P}(\mathcal{C} \mathcal{A T}) / / P$

4.6.3. Definition. Let $(P, U)$ be a strict operad in categories. We define the category $\mathcal{O P}_{\text {st }}(\mathcal{C} \mathcal{A T}) / / P$ as a subcategory of $\mathcal{O P}(\mathcal{C} \mathcal{A T}) / / P$, consisting of the same objects, but for any morphism $((F, \zeta), \eta, \alpha)$ in $\mathcal{O P}(\mathcal{C} \mathcal{A T}) / / P$, it is also a morphism in $\mathcal{O P}{ }_{s t}(\mathcal{C} \mathcal{A T}) / / P$ if $\zeta$ and $\eta$ are identities.

4.7. Operad-like triples as lax representations. So far we have considered categorical operads abstractly. In this subsection we will work with specific operads, namely the endomorphism operads of categories. Example 4.2.2 shows that for any category $\mathcal{C}, \mathfrak{E}(\mathcal{C})$ is a pseudo-operad. Mapping $E$ to the identity functor on $\mathcal{C}$ obviously defines a structure of an operad on $\mathfrak{E}(\mathcal{C})$.

In this Section we are interested in representations of categorical operads, i.e. with lax morphisms $P \rightarrow \mathfrak{E}(\mathcal{C})$. As Proposition 4.6.2 shows, such representations form a category. We will work with them a lot, so we introduce a special term.

4.7.1. Definition. A generalized triple on a category $\mathcal{C}$ is a coherent lax representation on it of a strict operad in categories $P$. The category of generalized triples on $\mathcal{C}$ will be denoted by $\mathfrak{T}(\mathcal{C})$.

To justify the term "generalized triple" we give the following example, which is illustrative but inessential in our considerations. It was considered in [Ben] Section 5.4 .

4.7.2. Example. Let $P$ be the strict operad $E$. A lax representation of $E$ on a category $\mathcal{C}$ is simply triple on $\mathcal{C}$ in the usual meaning of the term.

Indeed such representation consists first of a functor $F: E \rightarrow F u n(\mathcal{C}, \mathcal{C})$, which amounts to choosing a functor $T: \mathcal{C} \rightarrow \mathcal{C}$, secondly of a natural transformation $\zeta: T \circ T \rightarrow T$, thirdly of a natural transformation $\eta: I d_{\mathcal{C}} \rightarrow T$, such that the conditions stated in Definitions 4.5.2 and 4.3.2 are satisfied.

The condition spelled out in Definition 4.3.2 translates into associativity of $\zeta$, 
i.e. into commutativity of the following diagram

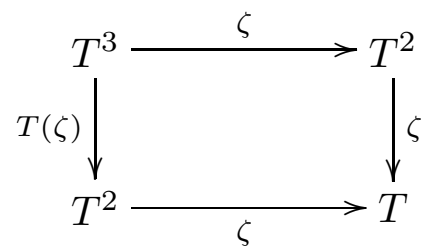

The condition stated in Definition 4.5.2 means that $\eta$ is a unit for the operation $\zeta$, i.e. the following diagram is commutative

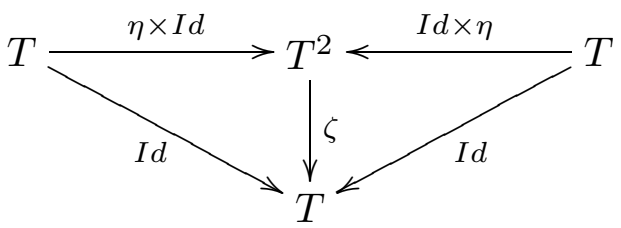

The main reason for the development of the theory of categorical operads, that we have done, is the notion of an "operad-like" triple on a category. As we have explained in introductory Section 4.0, we want to construct triples as colimits of a sequence of functors where each element of the sequence represents "operations of some arity".

However, in order for the combined object to satisfy the usual axioms of a triple, the individual elements should behave in a certain prescribed way with respect to colimits. So first we describe the conditions, which these individual functors should satisfy. Obviously we can consider colimits of any diagrams, but we will restrict our attention only to colimits of groupoids. Most of our results can be generalized to the case of arbitrary diagrams.

Consider a functor $F: \mathcal{C} \rightarrow \mathcal{C}$, and a diagram $D: \mathcal{D} \rightarrow \mathcal{C}$ in $C$. Since $F$ is a functor we have a natural transformation

$$
\chi_{F}: \operatorname{colim}(F \circ D) \rightarrow F(\operatorname{colim}(D)),
$$

where we consider both sides as functors from $\operatorname{Fun}(\mathcal{D}, \mathcal{C})$ to $\mathcal{C}$. We will say that a functor $F$ commutes with colimits of groupoids if $\chi_{F}$ is an isomorphism, whenever $\mathcal{D}$ is a groupoid.

In dealing with generalized triples we have a more general case of functors of the type $F: \mathcal{C}^{\times^{n}} \rightarrow \mathcal{C}$. We would like to extend the notion of commutativity with 
colimits to this case too. There is an obvious way to do that, namely we will say that $F$ commutes with colimits of groupoids if for every $1 \leq i \leq n$ and every $(n-1)$-tuple $\left\{C_{1}, . ., C_{i-1}, C_{i+1}, . ., C_{n}\right\}$ of objects of $\mathcal{C}$ the functor $F\left(C_{1}, . ., C_{i-1},-, C_{i+1}, . ., C_{n}\right)$ commutes with colimits of groupoids.

Having a functor $F: \mathcal{C}^{\times^{n}} \rightarrow \mathcal{C}$ that commutes with colimits of groupoids, we will encounter situations when we have $n$-diagrams $\left\{\mathcal{D}_{i}\right\}_{1 \leq i \leq n},\left\{D_{i}: \mathcal{D}_{i} \rightarrow \mathcal{C}\right\}$, and we will consider $F\left(D_{1}, \ldots, D_{n}\right)$. Since $F$ is a functor we have a morphism

$$
\operatorname{colim}\left(F\left(D_{1}, \ldots, D_{n}\right)\right) \rightarrow F\left(\operatorname{colim}\left(D_{1}\right), \ldots, \operatorname{colim}\left(D_{n}\right)\right)
$$

It is easy to see that this morphism factors through colimits in each variable of $F$, which are by assumption isomorphisms. Therefore this morphism is an isomorphism as well.

Remark. Our construction of generalized triples was specifically tailored for description of triples, i.e. monoids in the monoidal category of functors. However, since we work with operads in categories we obviously can use generalized triples to represent other objects, for example monoidal structures.

Indeed, consider a non-symmetric operad in categories, generated by one binary operation, and having isomorphisms in the category of ternary operations, connecting the two different ways of composing the binary operation with itself. If we demand that these isomorphisms satisfy the usual pentagon conditions of coherence, then a strict representation of this operad on a category is nothing else but a coherently associative product on this category. If we start with two generating operations and demand coherent associativity of both and in addition certain compatibility morphisms between their mixed compositions (these morphisms do not have to be isomorphisms), then a representation of such operad would be a 2 -monoidal category as described in [Va2].

Before we proceed with the definition of operad-like triples and provide a way of constructing ordinary triples from them, we need a technical preparation. We need to prove a lemma, that allows us to combine functors, that commute with colimits of groupoids, and get a functor commuting with such colimits as well. This will be needed in the proof of associativity of the structure natural transformation of the triple, that we construct from an operad-like one.

4.7.3. Lemma. Let $F, G$ be two functors $\mathcal{C} \rightarrow \mathcal{C}$, that commute with colimits of groupoids. Then F。G commutes with such colimits as well. Moreover, let $D^{\prime}: \mathcal{D}^{\prime} \rightarrow$ Fun $(\mathcal{C}, \mathcal{C})$ be a diagram of functors, commuting with colimits of groupoids, and $\mathcal{D}^{\prime}$ 
being a groupoid itself. Then for any groupoid $\mathcal{D}$ and any diagram $D: \mathcal{D} \rightarrow \mathcal{C}$ we have the following commutative diagram of natural transformations

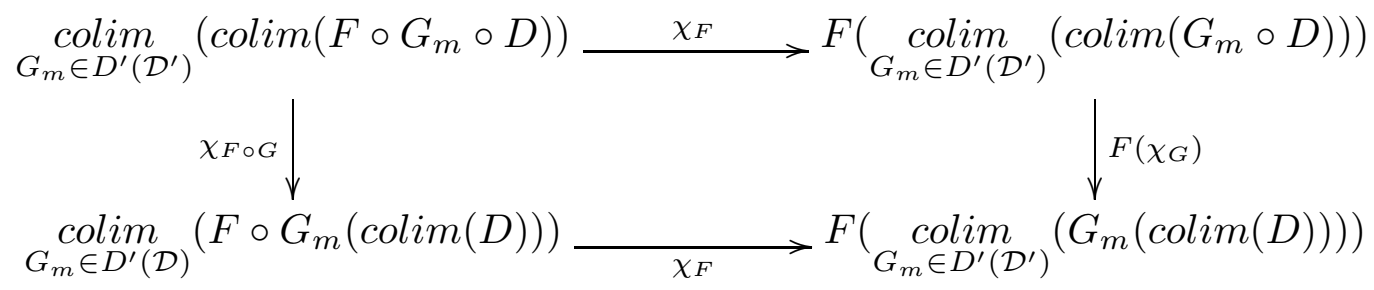

Proof. Let $C$ be in the image of $F \circ G \circ D$. There are two morphisms going out of $C$ : one to $F(\operatorname{colim}(G \circ D))$ and another one to $F \circ G(\operatorname{colim}(D))$. Since $F$ is a functor, $F\left(\chi_{G}\right)$ completes these morphisms to a commutative triangle. Therefore we have a factorization of the natural transformation $\operatorname{colim}(F \circ G \circ D) \rightarrow F \circ G(\operatorname{colim}(D))$ as $\chi_{F}$ applied to $G \circ D$, followed by $F\left(\chi_{G}\right)$. Both of these are isomorphisms, therefore so is their composition. This proves the first claim of the lemma.

The second claim is proved in a similar manner. One traces different ways to get from a object in the image of $D$ to $F\left(\underset{G_{m} \in D^{\prime}\left(\mathcal{D}^{\prime}\right)}{\operatorname{colim}}\left(G_{m}(\operatorname{colim}(D))\right)\right)$ and finds that they are equal, due to functoriality of $F$ and $G_{m}$ 's and the assumption that these functors commute with colimits of groupoids.

4.7.4. Definition. An operad-like triple on a category $\mathcal{C}$ is a generalized triple $P \rightarrow \mathfrak{E}(\mathcal{C})$, such that each component of $P$ is a groupoid and for each object of $P_{n}$ its image in Fun $\left(\mathcal{C}^{\times}, \mathcal{C}\right)$ commutes with colimits of groupoids.

We have defined the category $\mathfrak{T}(\mathcal{C})$ of all generalized triples on $\mathcal{C}$ by utilizing all possible morphisms of operads over an operad. With operad-like triples we want to restrict our attention to only strict subcategories as in definition 4.6.3.

We will denote by $\mathfrak{P}(\mathcal{C})$ the category whose objects are operad-like triples on $\mathcal{C}$ and whose morphisms are strict morphisms over $\mathfrak{E}(\mathcal{C})$ as defined in Definition 4.6.3.

4.7.5. Proposition. Let $((F, \zeta), \eta): P \rightarrow \mathfrak{E}(\mathcal{C})$ be an operad-like triple on $\mathcal{C}$. Then if we define a functor $\operatorname{Tot}(F): \mathcal{C} \rightarrow \mathcal{C}$ as follows

$$
\operatorname{Tot}(F)(C):=\coprod_{n \in \mathbb{Z}_{>0}} \underset{G_{n} \in F_{n}\left(P_{n}\right)}{\operatorname{colim}}\left(G_{n}\left(C^{\times^{n}}\right)\right)
$$

we get a triple on $\mathcal{C}$, with the multiplication and the unit given by $\zeta$ and $\eta$ respectively. In this way we get a functor Tot $: \mathfrak{P}(\mathcal{C}) \rightarrow \mathcal{T}(\mathcal{C})$ from the category of operad-like triples on $\mathcal{C}$ to the category of triples on it. 
Proof. First we give the definition of the multiplication and the unit natural transformations for $\operatorname{Tot}(F)$. The multiplication $\operatorname{Tot}(\zeta)$ is defined as composition of the following sequence of natural transformations:

$$
\begin{aligned}
& \coprod_{n \in \mathbb{Z}_{>0}} \underset{G_{n} \in F_{n}\left(P_{n}\right)}{\operatorname{colim}}\left(G_{n}\left(\left(\coprod_{m \in \mathbb{Z}>0} \underset{G_{m}^{\prime} \in F_{m}\left(P_{m}\right)}{\operatorname{colim}}\left(G_{m}^{\prime}\left(C^{\times^{m}}\right)\right)\right)^{\times^{n}}\right)\right) \rightarrow \\
\rightarrow & \coprod_{n, m_{1}, . ., m_{n}} \underset{G_{n}, G_{m_{i}}^{\prime} \in F(P)}{\operatorname{colim}}\left(G_{n}\left(G_{m_{1}}^{\prime}\left(C^{\times^{m_{1}}}\right), . ., G_{m_{n}}^{\prime}\left(C^{\times^{m_{n}}}\right)\right)\right) \rightarrow \\
\rightarrow & \coprod_{n, m_{1}, . ., m_{n}} \underset{G_{n}, G_{m_{i}}^{\prime} \in F(P)}{\operatorname{colim}}\left(F\left(G_{n} \circ\left(G_{m_{1}}^{\prime}, . ., G_{m_{n}}^{\prime}\right)\right)\left(C^{\times^{m_{1}+. .+m_{n}}}\right)\right),
\end{aligned}
$$

where the first arrow is given by commutativity of $G_{n}$ with colimits of groupoids and the second arrow is the sum of natural transformations $\zeta_{m_{1}, . ., m_{n}}$, given by the lax representation.

The unit $\operatorname{Tot}(\eta)$ is given as composition of the following sequence of natural transformations

$$
I d_{\mathcal{C}} \rightarrow F_{1}(e) \rightarrow \coprod_{n \in \mathbb{Z}_{>0}} \underset{G_{n} \in F_{n}\left(P_{n}\right)}{\operatorname{colim}}\left(G_{n}\right),
$$

where $e$ is the image under $F$ of the identity in $P$, the first arrow is given by the unit $\eta$ in the lax representation $((F, \zeta), \eta)$, and the second arrow is the natural inclusion of an object of a diagram into the colimit of the diagram.

We have to show that associativity and unit axioms hold. We know from definition of coherent lax morphisms of operads (Definition 4.3.2) that the natural transformation $\zeta$ satisfies associativity conditions. The multiplication for $\operatorname{Tot}(F)$ is given as a colimit of $\zeta$ 's, using commutativity with colimits of groupoids of individual functors in $F(P)$. From lemma 4.7.3 we know that different ways of composing $\chi$ 's for colimits of functors produce the same result, therefore from associativity of $\zeta$ follows associativity of $\operatorname{Tot}(\zeta)$. Similarly unit properties of $\eta$ with respect to $\zeta$ imply the same for $\operatorname{Tot}(\eta)$ with respect to $\operatorname{Tot}(\zeta)$.

It remains to show that $T o t$ is a functor from $\mathfrak{P}(\mathcal{C})$ to $\mathcal{T}(\mathcal{C})$. Given two operadlike triples $((F, \zeta), \eta): P \rightarrow \mathfrak{E}(\mathcal{C}),\left(\left(F^{\prime}, \zeta^{\prime}\right), \eta\right): P^{\prime} \rightarrow \mathfrak{E}(\mathcal{C})$ and a morphism $\left(F^{\prime \prime}, \alpha\right)$ from the first to the second we have a natural transformation $\operatorname{Tot}(\alpha): \operatorname{Tot}(F) \rightarrow$ $\operatorname{Tot}\left(F^{\prime}\right)$, given by $\alpha$. Indeed, each $G \in F(P)$ is mapped by $\alpha$ to $F^{\prime}\left(F^{\prime \prime}(G)\right)$ in $F^{\prime}\left(P^{\prime}\right)$. The latter is canonically included into $\operatorname{Tot}\left(F^{\prime}\right)$.

Now we see that the compatibility conditions for $\alpha$ with $\zeta, \zeta^{\prime \prime}$ and with $\eta, \eta^{\prime}$ translate exactly to the fact that $\operatorname{Tot}(\alpha)$ is a map between monoids in the monoidal category of endofunctors on $\mathcal{C}$. 
4.8. Symmetric operad-like triples. So far we have dealt with non-symmetric operads, and therefore with non-symmetric operad-like triples. Now we would like to introduce action of symmetric groups in our construction. Since the monoidal category $(\mathcal{C} \mathcal{A} \mathcal{T}, \times, E)$ is symmetric there is a standard notion of a symmetric strict operad in categories.

4.8.1. Definition. A symmetric strict operad in categories is a classical symmetric operad (e.g. [MarShSt], Section 1.2) in the category $(\mathcal{C} \mathcal{A} \mathcal{T}, \times, E)$, i.e. it is a strict operad $P$ and for each $n \in \mathbb{Z}_{>0}$ an action of the symmetric group $\Sigma_{n}$ on the category $P_{n}$ is given. We will denote the functor on $P_{n}$, that corresponds to an element $\sigma_{n} \in \Sigma_{n}$, by the same symbol $\sigma_{n}$. A coherent lax morphism between two symmetric strict operads is a coherent lax morphism between the operads (as defined in Definition 4.5.2), such that the functors $F_{n}$ commute (on the nose) with the action of symmetric groups.

Action of symmetric groups by means of functors provides definition of a symmetric operad in categories, but it is not useful for defining symmetric operad-like triples, since we need natural transformations for that. Therefore we introduce the notion of an equivariant symmetric operad in categories.

In order to do that we need more notation. We will denote by $\underline{\Sigma}_{n}$ the category whose objects are elements of the symmetric group $\Sigma_{n}$ and the set of morphisms $\operatorname{Hom}\left(\sigma_{n}, \sigma_{n}^{\prime}\right)$ between any two of them consists of one element: $\sigma_{n}^{-1} \sigma_{n}^{\prime} \in \Sigma_{n}$. Composition is obvious. One could call this "a regular groupoid" version of the symmetric group $\Sigma_{n}$.

4.8.2. Definition. An equivariant symmetric operad in categories is a symmetric strict operad $P$, such that for every object $p_{n} \in P_{n}$ there is a functor $S_{n}: \underline{\Sigma}_{n} \rightarrow P_{n}$, such that any object $\sigma_{n} \in \underline{\Sigma}_{n}$ is mapped to $\sigma_{n}\left(p_{n}\right)$, and the following compatibility conditions are satisfied. Let $p_{m_{i}} \in P_{m_{i}} 1 \leq i \leq n$. Then for every $(n+1)$-tuple of morphisms $\sigma_{n} \in \underline{\Sigma}_{n}, \sigma_{m_{i}} \in \underline{\Sigma}_{m_{i}}$, the composition functor $\Upsilon_{m_{1}, \ldots, m_{n}}: P_{n} \times P_{m_{1}} \times \cdots \times P_{m_{n}} \rightarrow P_{m_{1}+\cdots+m_{n}}$ maps $S_{n}\left(\sigma_{n}\right) \times I d^{\times^{n}}$ to $S_{m_{1}+\cdots+m_{n}}\left(\sigma_{\underline{n}}\right)$, where the morphism $\sigma_{\underline{n}} \in \underline{\Sigma}_{m_{1}+\cdots+m_{n}}$ is $\sigma_{n}$-permutation of the $n$ blocks. Also the composition functor maps $I d \times S_{m_{1}}\left(\sigma_{m_{1}}\right) \times \cdots \times S_{m_{n}}\left(\sigma_{m_{n}}\right)$ to $S_{m_{1}+\cdots+m_{n}}\left(\sigma_{m_{1}} \times \cdots \times \sigma_{m_{n}}\right)$, where the morphism $\sigma_{m_{1}} \times \cdots \times \sigma_{m_{n}} \in \underline{\Sigma}_{m_{1}+\cdots+m_{n}}$ corresponds to the product of permutations.

It is clear that the compatibility conditions in the last definition are meant to reflect the standard equivariance properties of operads in symmetric categories. Indeed, when we will define symmetric operad-like triples we will see that these compatibility conditions translate into the usual equivariance. 
4.8.3. Example. Let $\mathcal{C}$ be a category and consider the endomorphism operad $\mathfrak{E}(\mathcal{C})$ of $\mathcal{C}$ (Example 4.2.2.). There is one obvious symmetric structure on $\mathfrak{E}(\mathcal{C})$, namely for any $\sigma_{n} \in \Sigma_{n}$ we define a functor $\sigma_{n}: F u n\left(\mathcal{C}^{\times n}, \mathcal{C}\right) \rightarrow F u n\left(\mathcal{C}^{\times}, \mathcal{C}\right)$ as follows

$$
\operatorname{Fun}\left(\mathcal{C}^{\times^{n}}, \mathcal{C}\right) \ni F \mapsto F \circ \sigma_{n}
$$

where we consider $\sigma_{n}$ as a functor $\mathcal{C}^{\times^{n}} \rightarrow \mathcal{C}^{\times^{n}}$, permuting the variables. It is clear that in this way we get a symmetric structure on $\mathfrak{E}(\mathcal{C})$ and we will always consider endomorphism functors with symmetric structures chosen in this way.

Note that in general an endomorphism operad is not an equivariant symmetric operad. However, we do not require equivariance in the definition of a lax morphism between two symmetric operads in categories, and consequently we can consider lax morphisms from an equivariant operad to one which is not. When the codomain is an endomorphism operad as in the last example we will have a special name for it.

4.8.4. Definition. We will call a lax morphism from an equivariant symmetric operad $P$ to an endomorphism operad $\mathfrak{E}(\mathcal{C})$ a symmetric generalized triple. If every component of $P$ is a groupoid and every functor in the image of the generalized triple commutes with colimits of groupoids, we will call such a generalized triple a symmetric operad-like triple. We organize symmetric operad-like triples into a category, where morphisms are lax morphisms as in Definition 4.7.4, and in addition commuting (on the nose) with the symmetric structure (as in Definition 4.8.1.) We will denote this category by $\mathfrak{P}^{s}(\mathcal{C})$.

We have proved a proposition (Proposition 4.7.7) stating that we can get a usual triple from an operad-like one, and that this correspondence is a functor. Since equivariant symmetric operads differ from the non-symmetric ones by presence of an action of symmetric groups (functors) and representations of the "regular symmetric groupoids" (invertible morphisms) we see that the same proof applies to equivariant operad-like triples as well. So we get a functor $T$ ot $: \mathfrak{P}^{s}(\mathcal{C}) \rightarrow \mathcal{T}(\mathcal{C})$.

We would like to illustrate the role of equivariance in representation of an equivariant symmetric operad on a category. So let $P$ be an equivariant symmetric operad. Let $\mathcal{C}$ be a category and let $((F, \zeta), \eta)$ be a representation of $P$ on $\mathcal{C}$. Let $p_{n},\left\{p_{m_{i}}\right\}_{1 \leq i \leq n}$ be elements of $P_{n}$ and $\left\{P_{m_{i}}\right\}_{1 \leq i \leq n}$ respectively. Let $\sigma_{n},\left\{\sigma_{m_{i}}\right\}$ be morphisms in $\underline{\Sigma}_{n}$ and $\left\{\underline{\Sigma}_{m_{i}}\right\}$. Writing explicitly the conditions for $\zeta$ to be a natural transformation we get the following commutative diagrams of natural transforma- 
tions between functors on $\mathcal{C}$

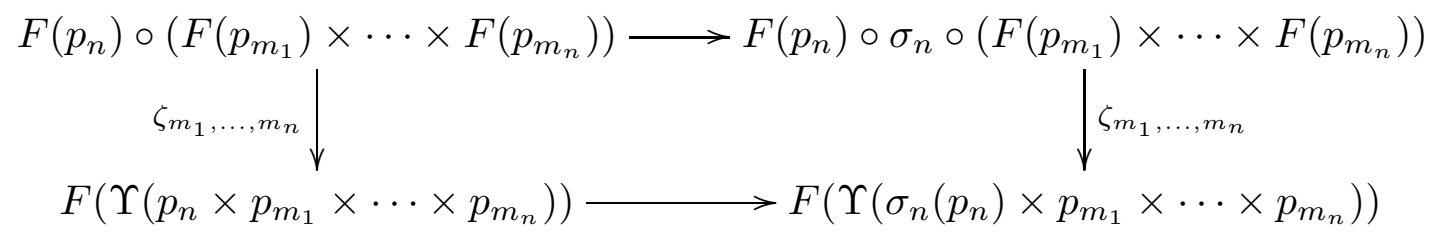

where the upper horizontal arrow is $F\left(S\left(\sigma_{n}\right)\right) \circ\left(I d^{\times^{n}}\right)$, and the lower horizontal arrow is $F\left(S\left(\sigma_{\underline{n}}\right)\right)$.

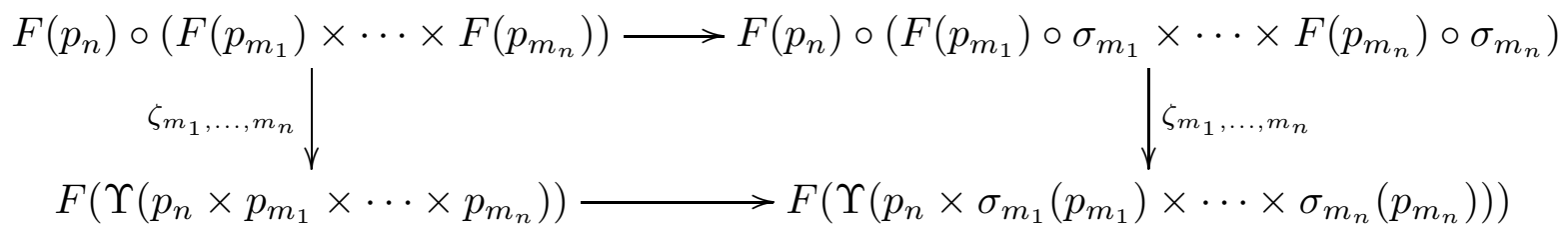

where the upper horizontal arrow is $\operatorname{Id} \circ\left(F\left(S\left(\sigma_{m_{1}}\right) \times \cdots \times S\left(\sigma_{m_{n}}\right)\right)\right.$, and the lower horizontal arrow is $F\left(S\left(\sigma_{m_{1}} \times \cdots \times \sigma_{m_{n}}\right)\right)$.

When we apply $T$ ot to a symmetric operad-like triple we see that these diagrams translate to the usual equivariance diagrams for operads.

4.9. Example: operads as algebras over symmetric operad-like triples. In this subsection we would like to show that operads, as they were defined in Section 1, can be described as algebras over certain triples, that lie in the image of Tot, as described above. Until now we have considered operad-like triples as lax representations of classical operads. However, operadic constructions require working with colored operads, rather than the classical ones. The passage to the colored context is straightforward and we indicate the main steps below.

One could define colored operads in categories as colored operads in the monoidal category $(\mathcal{C} \mathcal{A} \mathcal{T}, \times, E)$ in the usual meaning of the term. However, because we have 2 -morphisms in the background, there are some minor adjustments to be made. As in the usual case, a colored operad is different from a classical one by a restriction on possible compositions. Before we define it we need a technical preparation.

Suppose we have three categories $\mathcal{C}, \mathcal{C}^{\prime}, \mathcal{C}^{\prime \prime}$, and each object in all of them is given two colors from a set of colors $\Omega$. One of the colors will be called incoming and the other outgoing. We will say that we have a colored functor $\mathcal{C} \times \mathcal{C}^{\prime} \rightarrow \mathcal{C}^{\prime \prime}$ if for every two objects $C, C^{\prime}$ of $\mathcal{C}$ and $\mathcal{C}^{\prime}$ respectively, such that the incoming color 
of $C$ is equal to the outgoing one of $C^{\prime}$ (we will call such pairs composable), we are given an object $C^{\prime \prime}$ of $\mathcal{C}^{\prime \prime}$ whose incoming color is that of $C^{\prime}$ and outgoing - that of $C$. On morphisms such a functor should act as a usual functor, where we allow morphisms only between composable pairs in $\mathcal{C} \times \mathcal{C}^{\prime}$.

4.9.1. Definition. Let $\Omega$ be a set. A strict $\Omega$-colored categorical operad is a sequence of categories $\left\{P_{n}\right\}_{n \in \mathbb{Z}_{>0}}$, for each object in $P_{n}$ a set of incoming colors $\left(\omega_{1}, \ldots, \omega_{n}\right)$ and an outgoing color $\omega$, and a set of colored functors $\left\{\Upsilon_{m_{1}, \ldots, m_{n}}\right.$ : $\left.P_{n} \times P_{m_{1}} \times \cdots \times P_{m_{n}} \rightarrow P_{m_{1}+\cdots+P_{m_{n}}}\right\}$, satisfying the obvious associativity and unit axioms.

Just as in case of monochrome operads we can introduce the notion of a symmetric colored operad in categories. For that we need an action of symmetric groups on the components. However, since we need to be able to permute different sets of incoming colors differently we have to use colored symmetric groups, i.e. for every set of incoming colors we have a copy of the symmetric group, that acts (by functors) on all objects with the same set of incoming colors. We omit writing explicitly the colored extension of the usual equivariance axioms (it is straightforward but long).

Similarly the equivariance structure (Definition 4.8.2) can be generalized to the colored context in a very straightforward manner. Indeed it requires connecting objects by isomorphisms with their images under permutation functors, such that the compatibility conditions from Definition 4.8.2. are satisfied. We leave writing the details explicitly to the reader.

Let $\mathcal{C}$ and $\mathcal{C}^{\prime}$ be two categories. Suppose that objects in $\mathcal{C}$ have incoming and outgoing colors from a set of colors $\Omega$ and objects in $\mathcal{C}^{\prime}$ - from $\Omega^{\prime}$. Suppose we have a map $f: \Omega \rightarrow \Omega^{\prime}$. Then we will say that a functor $F: \mathcal{C} \rightarrow \mathcal{C}^{\prime}$ preserves colors if an object $C \in \mathcal{C}$ with colors $\left(\omega_{\text {in }}, \omega_{\text {out }}\right)$ is mapped by $F$ to an object $C^{\prime} \in \mathcal{C}^{\prime}$ with colors $\left(f\left(\omega_{\text {in }}\right), f\left(\omega_{\text {out }}\right)\right)$.

4.9.2. Definition. A coherent lax morphism between symmetric strict colored operads in categories (colored by $\Omega$ and $\Omega^{\prime}$ ) is a set of color preserving functors (for a choice of a map $\left.f: \Omega \rightarrow \Omega^{\prime}\right)$ and natural transformations $((F, \zeta), \eta)$, satisfying the colored versions of coherence conditions as in definitions 4.3.2 and 4.5.2.

4.9.3. Example. The main example for us of a colored categorical operad is the operad, produced by abstract categories of labelled graphs, described in Section 1. Let $\Gamma$ be a category as in Definition 1.3. Let $\Omega$ be the set of $\Gamma$-corollas. This is our set of colors.

We define a strict $\Omega$-colored operad $\mathfrak{G}$ as follows. We set $\mathfrak{G}_{n}$ to be the category, 
whose objects are pairs of morphisms in $\Gamma$ :

$$
\coprod_{v \in V_{\tau}} \sigma_{v} \rightarrow \tau \rightarrow \sigma
$$

where $\tau$ is an object of $\Gamma, \sigma$ is a $\Gamma$-corolla, and the first arrow is one of the possible atomizations of $\tau$, provided by property (iv) of Definition 1.3. We will denote such object simply by $\tau$. Note that corollas in the direct product are ordered.

A morphism from one such object to another is a pair of morphisms between $\tau$ 's and coproducts of corollas, such that together with the identity on $\sigma$ they make up a commutative ladder.

The coloring on each object of $\mathfrak{G}_{n}$ is obvious: as it is written above the outgoing color is $\sigma$ and the incoming colors are $\left\{\sigma_{v}\right\}_{v \in V_{\tau}}$. Actions by symmetric groups are obvious as well: we just rearrange summands in the direct sum of corollas for the atomization.

To define composition functors we use property (vi) of Definition 1.3. Suppose we have $n+1$ objects of $\mathfrak{G}: \tau_{1}, \ldots, \tau_{n}, \tau$, such that the incoming colors of the latter are exactly the outgoing ones of the former $n$-tuple. According to property (vi) we have an object $\tau^{\prime}$ of $\Gamma$, and a morphism $\tau^{\prime} \rightarrow \tau$, fitting into diagram of the type (1.3). Here we assume that a choice of a particular $\tau^{\prime}$ is made in each case. We will call this a choice of grafting. It is clear that we can choose atomization of $\tau^{\prime}$ to be the direct sum of atomizations of $\tau_{i}$ 's. Doing that and taking $\tau^{\prime} \rightarrow \tau \rightarrow \sigma$ as the outgoing color we get a composition on $\mathfrak{G}$. Colored units are chosen in the obvious way: they are identity maps of the corollas.

Now we have to check the associativity and unit axioms. The unit ones are obvious. Associativity conditions are obvious if we take $G r$ itself as $\Gamma$, indeed all we do is substituting graphs in place of corollas, and this operation is associative. In case of a general $\Gamma$ we make this associativity condition part of the choice of grafting.

Actions of symmetric groups obviously satisfy the conditions of Definition 4.8.1. We also have a natural equivariance structure (Definition 4.8.2) on $\mathfrak{G}$. Indeed if we take a direct sum and rearrange the summands the result is connected to the original sum by a unique isomorphism, that gives us the representation of $\underline{\Sigma}$. Here we should check that compatibility conditions from 4.8 .2 are satisfied. In case $\Gamma$ is $G r$ itself they are obvious. In general we make them part of the requirements for the choice of grafting. So we have a structure of an equivariant symmetric operad on $\mathfrak{G}$.

Now let $(\mathcal{C}, \otimes)$ be a symmetric monoidal category. We would like to define a 
representation of $\rho: \mathfrak{G} \rightarrow \mathfrak{E}(F u n(\Omega, \mathcal{C}))$ as follows: given an object of $\mathfrak{G}$

$$
\coprod_{1 \leq i \leq n} \sigma_{i} \rightarrow \tau \rightarrow \sigma
$$

and an object $F: \Omega \rightarrow \mathcal{C}, \rho(\tau)$ acts on $F$ by mapping it to the functor $\Omega \rightarrow \mathcal{C}$, whose value on $\sigma$ is $\bigotimes_{1 \leq i \leq n} F\left(\sigma_{i}\right)$ and on the rest of colors the value is the initial object of $\mathcal{C}$.

If we did not have non-identity morphisms on $\Omega$, then this definition would have been obviously correct. Indeed, then a functor from the category of colors would have been equivalent to just a choice of objects in $\mathcal{C}$, and the above choice is obviously functorial in $F$. However, we have to take into account the non-identity morphisms in $\Omega$.

Let $\sigma$ and $\sigma^{\prime}$ be two corollas. And let $\phi: \sigma \rightarrow \sigma^{\prime}$ be an isomorphism. Then for any object $\coprod_{1 \leq i \leq n} \sigma_{i} \rightarrow \tau \rightarrow \sigma$ of $\mathfrak{G}$ we have a new object $\coprod_{1 \leq i \leq n} \sigma_{i} \rightarrow \tau \rightarrow \sigma^{\prime}$, where the last arrow is the last arrow in the original object, followed by $\phi$. We will denote this new object of $\mathfrak{G}$ by $\phi_{*}(\tau)$. In this way, given an object of $\mathfrak{G}$ we get a sort of $\Omega$-diagram of such objects (it is not exactly a diagram because we have excluded morphisms from $\mathfrak{G}$, that are non-identities on the corollas).

Note that for any isomorphism $\phi$ as above the values of $\rho(\tau)(F)$ on $\sigma$ and of $\rho\left(\phi_{*}(\tau)\right)(F)$ on $\sigma^{\prime}$ are the same. Therefore, if given a $F$, a $\tau \in \mathfrak{G}$ we define for each corolla $\sigma^{\prime} \in \Omega$, that is isomorphic to $\sigma$

$$
\sigma^{\prime} \mapsto \coprod \bigotimes_{1 \leq i \leq n} F\left(\sigma_{i}^{\prime}\right)
$$

where the coproduct is taken over all objects from $\mathfrak{G}$, that are in the $\Omega$-diagram corresponding to $\tau$ as described above, then we would get a new functor $\Omega \rightarrow$ $\mathcal{C}$. Indeed, every morphism in $\Omega$ (as for example $\phi$ ) is mapped to the identity automorphism of $\bigotimes_{1 \leq i \leq n} F\left(\sigma_{i}\right)$.

So we get a representation of $\mathfrak{G}$ on $\operatorname{Fun}(\Omega, \mathcal{C})$, and we will denote it by $\rho$. As it was noted above $\mathfrak{G}$ is an equivariant symmetric operad and hence the operad-like triple $\rho$ is symmetric.

From Proposition 4.7.5 we conclude that there is a triple $\operatorname{Tot}(\rho)$ on $\operatorname{Fun}(\Omega, \mathcal{C})$. This triple is exactly the triple $\mathcal{F}$ from Section 1.5.4, and the algebras over it are the $\Gamma \mathcal{C}$-operads. 
4.10. Existence of $\underline{\text { cohom }}$ for operads in algebras. Let $T$ be a Hopf-like triple (Definition 3.1.1) on a category $\mathcal{C}$, that commutes with colimits of groupoids. From Lemma 3.1.3 we know that the category $\mathcal{A}$ of algebras over $T$ has monoidal structure, and hence for any abstract category of labelled graphs $\Gamma$ we can consider the category $\Gamma \mathcal{A O P E R}$ of $\Gamma \mathcal{A}$-operads. Now we would like to show that if $\mathcal{C}$ possesses cohom, so does $\Gamma \mathcal{A O P E R}$.

The key to the proof is the observation that the forgetful functor $\mathfrak{U}: \mathcal{A} \rightarrow \mathcal{C}$ maps $\Gamma \mathcal{A}$-operads to $\Gamma \mathcal{C}$-operads. Therefore an object of $\Gamma \mathcal{A O P E R}$ is a sequence (parameterized by $\Gamma$-corollas) of objects in $\mathcal{C}$, such that each one of them is a $T$-algebra and altogether they make up a $\Gamma$-operad in $\mathcal{C}$. Of course certain compatibility conditions between these two structures should be satisfied. This situation is just a triple version of the usual instance of an action of a colored operad.

First we are going to consider sequences of objects in $\mathcal{C}$, that have both of the above structures, but with the compatibility conditions omitted. We need a very simple lemma for this, whose proof is straightforward and we leave it to the reader.

4.10.1. Lemma. Let $T$ and $T^{\prime}$ be two triples on $\mathcal{C}$, such that both commute with colimits of groupoids. Then the category $\mathcal{A}^{\prime \prime}$ of objects in $\mathcal{C}$, that are simultaneously algebras over $T$ and $T^{\prime}$ is equivalent to the category of algebras over the following triple:

$$
T \coprod T^{\prime}: C \mapsto \coprod T\left(\ldots T^{\prime}(\ldots(C))\right),
$$

where the coproduct is taken over all possible words of positive length, composed of $T$ and $T^{\prime}$.

In our situation we have one $T$ (one for each $\Gamma$-corolla) but instead of $T^{\prime}$ we have a sequence (parameterized by objects of $\mathfrak{G}$ ) of functors $\mathcal{C}^{x^{n}} \rightarrow \mathcal{C}$ (for all $n \in \mathbb{Z}_{>0}$ ). Each of these functors commutes with colimits of groupoids so we can form a triple out of them and $T$ by forming all possible compositions and summing them up. This is an obvious generalization of Lemma 4.10.1. We will denote the resulting triple by $T \coprod \operatorname{Tot}(\rho)$.

By construction $T \coprod \operatorname{Tot}(\rho)$ is the direct product, i.e. its algebras are equivalent to operads in $\mathcal{C}$ and algebras over $T$, and these two structures being unrelated. Now we make this direct product into an amalgamated sum. The needed relations are provided by the Hopf-like properties of $T$. Recall that for $T$ to be Hopf-like means that there is a natural transformation

$$
\tau: T \circ \otimes \rightarrow \otimes \circ(T \times T),
$$

satisfying certain conditions, spelled out in Definition 3.1.1. 
According to property b) in Definition 3.1.1. there is a definite natural transformation

$$
T \circ(\otimes \circ(I d \times \otimes)) \rightarrow \otimes \circ(I d \times \otimes) \circ\left(T^{\times^{3}}\right),
$$

and similarly for all other possible iterations of the monoidal structure. Note that functors on both sides of the last arrow are summands in $T \coprod \operatorname{Tot}(\rho)$ (for each $\Gamma$-corolla), therefore there are two ways to include the left side in the sum, i.e. we have a pair of parallel natural transformations (for each $\Gamma$-corolla separately):

$$
\coprod_{n \geq 1} T \circ \otimes^{\circ^{n}} \rightrightarrows T \coprod T o t(\rho)
$$

These are our relations. The left side of the two arrows is just a functor, but we have an adjunction from functors (commuting with coproducts) to triples, therefore we have a pair of morphisms between triples

$$
\mathfrak{F}\left(\coprod_{n \geq 1} T \circ \otimes^{\circ^{n}}\right) \rightrightarrows T \coprod T o t(\rho)
$$

where $\mathfrak{F}$ denotes the free triple. The coequalizer (in the category of triples) of these two morphisms is a triple whose algebras are exactly $\Gamma$-operads in $\mathcal{A}$.

Here we should discuss existence of coequalizers in the category of triples on $\mathcal{C}$. The opposite category of $\operatorname{Fun}(\mathcal{C}, \mathcal{C})$ is equivalent to $F u n\left(\mathcal{C}^{o p}, \mathcal{C}^{o p}\right)$, and hence the question of existence of colimits in $\operatorname{Fun}(\mathcal{C}, \mathcal{C})$ is equivalent to the question of existence of limits in $F u n\left(\mathcal{C}^{o p}, \mathcal{C}^{o p}\right)$. The latter can be answered by existence of colimits in $\mathcal{C}$ (e.g. [Bo1], Proposition 2.15.1.) Thus if we assume that $\mathcal{C}$ has coequalizers, so does $F u n(\mathcal{C}, \mathcal{C})$. So the question of existence of coequalizers in the category of triples on $\mathcal{C}$ is the usual question of lifting colimits from a category to a category of algebras over a triple. General conditions for their existence are very restrictive, so we will assume existence of the coequalizers above as a condition imposed on $\mathcal{C}$ itself and on $T$.

So far we have considered algebras over a triple, that commutes with colimits of groupoids. However, it is not always the case, as for example the triple of associative algebras in vector spaces does not commute with such colimits (in general it does not commute even with coproducts). Yet often triples that do not commute can be represented as colimits of ones, which do commute, such as the operad-like triples.

We need a reformulation of the property of a triple to be Hopf-like in the language of operad-like triples. The following definition expresses in the operad-like triple 
setting the property of an operad to be Hopf. This notion is well known, so we will only outline the main parts of the definition and omit the necessary coherence properties. Examples of such operad-like triples are provided by Hopf operads.

4.10.2. Definition. Let $F: P \rightarrow \mathfrak{E}(\mathcal{C})$ be an operad-like triple on $\mathcal{C}$. It is Hopf-like if for every $G_{n} \in F_{n}\left(P_{n}\right), n \in \mathbb{Z}_{>0}$ we have a natural transformation

$$
\tau_{n}: G_{n} \circ \otimes^{\times^{n}} \rightarrow \otimes \circ\left(G_{n} \times G_{n}\right) \circ \sigma_{2 n}
$$

where $\sigma_{2 n}$ is the permutation that moves all elements in the even places of a sequence to the end of it. This natural transformation should satisfy coherence conditions expressing its associativity and compatibility with the composition natural transformation on $F(P)$.

Now assume that we have a Hopf-like symmetric operad-like triple $F: P \rightarrow$ $\mathfrak{E}(\mathcal{C})$. The family $\left\{\tau_{n}\right\}$ provides us with a Hopf-like structure on $\operatorname{Tot}(F)$. Thus the category $\mathcal{A}$ of algebras over $\operatorname{Tot}(F)$ has a symmetric monoidal structure. Let $\Gamma$ be an abstract category of labelled graphs. We would like to have objects of $\Gamma \mathcal{A O P E R}$ as algebras over a triple on $\mathcal{C}$.

Just as we did in case of a single $T$ we first consider the coproduct $\operatorname{Tot}(F) \coprod \operatorname{Tot}(\rho)$. Here, as before, we take all possible compositions, but now we have to compose functors in several variables. Also we want to take coproduct of symmetric operad-like triples, i.e. we add up not only all compositions, but also applications to them of permutations of variables.

Finally, as before, we have a pair of parallel morphisms of triples, with codomain $\operatorname{Tot}(F) \coprod \operatorname{Tot}(\rho)$. Their coequalizer (if it exists) is the required triple. In total we have the following proposition.

4.10.3. Proposition. Let $(\mathcal{C}, \otimes)$ be a symmetric monoidal category. Let $F$ : $P \rightarrow \mathfrak{E}(\mathcal{C})$ be a symmetric operad-like triple on $\mathcal{C}$. Suppose that $F$ is Hopf-like, and let $\Gamma$ be an abstract category of labelled graphs. Then, if the category of triples on $\mathcal{C}$ has necessary coequalizers, the category of $\Gamma$-operads in the category of algebras over $\operatorname{Tot}(F)$ is equivalent to a category of algebras over a triple on $\mathcal{C}$.

Now using results of Section 3.1. we establish existence of cohom for operads in algebras over operad-like triples, given that cohom exists on $\mathcal{C}$. 


\section{Appendix. Labeled graphs corresponding to various operads.}

This will last out a night in Russia

When nights are longest there.

W.Shakespeare, Measure for measure, 2.1.132-3

0. Operads, cyclic operads, modular operads. The graph geometry behind these structures is basically well known, and we will only briefly repeat it.

Operads. Objects: disjoint unions of directed trees with one output each. Morphisms: (generated by) contractions and graftings of an output to an input. If one considers only linear directed graphs (each vertex carries one input and one output), one gets associative algebras.

Cyclic operads. Objects: disjoint unions of (unlabeled) trees. Morphisms: contractions and graftings.

If one adds cyclic labeling, one gets the non-symmetric version of operads, resp. cyclic operads.

Modular operads. Objects: graphs of arbitrary topology with genus labeling. Morphisms: contractions and graftings compatible with labelings in the following sense.

Contraction of an edge having two distinct vertices of genera $g_{1}, g_{2}$, produces a new vertex of genus $g_{1}+g_{2}$. Contraction of a loop augments the genus of its vertex by one. The effect of a general contraction is the result of the composition of contraction of edges. Grafting does not change labels.

1. PROPs. Consider first the category $\Gamma_{c}$ whose objects are disjoint unions of oriented corollas, and morphisms are mergers (including isomorphisms). Any tensor functor $\left(\Gamma_{c}, \coprod\right) \rightarrow(\mathcal{G}, \otimes)$ is determined up to an isomorphism by the following data:

(i) Its values on corollas with inputs $\{1, \ldots, n\}$ and outputs $\{1, \ldots, m\}(m=0$ and $n=0$ are allowed). Let such a value be denoted $P(m, n)$.

(ii) Its values upon automorphisms of such corollas. This means that each $P(m, n)$ is endowed by commuting actions of $\mathbf{S}_{m}$ (left) and $\mathbf{S}_{n}$ (right).

(iii) Its values upon merger morphisms of such corollas which are called horizontal compositions:

$$
P\left(m_{1}, n_{1}\right) \otimes \cdots \otimes P\left(m_{r}, n_{r}\right) \rightarrow P\left(m_{1}+\cdots+m_{r}, n_{1}+\cdots+n_{r}\right) .
$$


Consider now a larger category $\Gamma$ of directed graphs without oriented wheels. This puts restrictions to morphisms compatible with orientations. In particular, if we contract an edge, we must simultaneously contract all edges connecting its ends. Mergers of two vertices connected by an oriented path also are excluded.

A tensor functor $\Gamma \rightarrow \mathcal{G}$ then produces data (i)-(iii) and moreover,

(iv) Vertical compositions: values of the functor upon full contractions of twovertex directed graphs such that all inputs belong to one vertex, all outputs to another, and edges are oriented from inputs to outputs:

$$
P(m, n) \otimes P(n, k) \rightarrow P(m, k), n \neq 0 .
$$

These data must satisfy some compatibility conditions which can be rephrased as existence of a monoidal category with objects $\emptyset, \ldots,\{1, \ldots, n\}, \ldots$ (as in 1.2.3) enriched over $\mathcal{G}$ in such a way that its morphisms become $P(m, n)$ and their composition is given by (A.2).

Allowing mergers in PROPs, we get generally big categories $\Rightarrow \sigma$ which are main building blocks of the triple $(\mathcal{F}, \mu, \eta)$ and the respective operads. In the following three operadic structures, we again exclude them.

2. Properads. Objects: all directed graphs as above. Morphisms: contractions and graftings.

3. Dioperads. Objects: all directed graphs with whose connected components are simply connected. Morphisms: contractions and graftings.

4. $\frac{1}{2}$-PROPs. Objects: directed graphs with simply connected components trees such that each edge is either unique output of its source, or unique input of its target. Morphisms: contractions and graftings.

5. Monoidal structures on the collections. Following [Va1], we will introduce the following definition, working well for the categories of directed graphs without mergers.

A directed graph $\tau$ is called two-level one, if there exists a partition of its vertices $V_{\tau}=V_{\tau}^{1} \amalg V_{\tau}^{2}$ such that

a) Tails at $V_{\tau}^{1}$ are all inputs of $\tau$, tails at $V_{\tau}^{2}$ are all outputs of $\tau$.

b) Any edge starts at $V_{\tau}^{1}$ and ends at $V_{\tau}^{2}$.

Clearly, such a partition is unique, if it exists at all.

Denote by $\Rightarrow^{(2)} \sigma$ the full subcategory of $\Rightarrow \sigma$ consisting of objects whose sources are two-level graphs. 
For any two collections $A^{1}, A^{2}$, define the third one by

$$
\left(A^{2} \bigotimes_{c} A^{1}\right)(\sigma):=\operatorname{colim}\left(\otimes_{v \in V_{\tau}^{1}} A^{1}\left(\tau_{v}\right)\right) \otimes\left(\otimes_{v \in V_{\tau}^{2}} A^{2}\left(\tau_{v}\right)\right)
$$

where colim is taken over $\Rightarrow^{(2)} \sigma$.

B. Vallette proves that this is a monoidal structure on collections, and that the respective operads are monoids in the resulting monoidal category.

B. Vallette treats also the case of PROPs, but here one must restrict oneself to "saturated" collections.

\section{References}

[BarW] M. Barr, Ch. Wells. Toposes, triples and theories. Grundlehren der mathematischen Wissenschaften 278, Springer Verlag, 1985, 358 pp.

[Bat] M. Batanin. The Eckmann-Hilton argument, higher operads and $E_{n}-$ spaces. math.CT/0207281 Sep 2003, 58 pp.

[Bek] T. Beke Sheafifiable homotopy model categories. II. J. Pure Appl. Algebra 164 (2001), no. 3, 307-324.

[Ben] J. Bénabou. Introduction to bicategories in 1967 Reports of the Midwest Category Seminar, Springer Verlag, 1-77.

[BeMa] K. Behrend, Yu. Manin. Stacks of stable maps and Gromov-Witten invariants. Duke Math. Journ, vol. 85, No. 1 (1996), 1-60.

[BerDW] R. Berger, M. Dubois-Violette, M. Wambst. Homogeneous algebras. J. Algebra, 261 (2003), 172-185. Preprint math.QA/0203035

[BerM] R. Berger, N. Marconnet. Koszul and Gorenstein properties for homogeneous algebras. Algebras and representation theory, 9 (2006), 67-97.

[BerMo] C. Berger, I. Moerdijk. Resolution of colored operads and rectification of homotopy algebras. math.AT/0512576 (to appear in Cont. Math., vol. in honor of Ross Street.)

[Bl] D. Blanc. New model categories from old. Journal of Pure and Applied Algebra 109 (1996), 37-60

[Bo1] F. Borceux. Handbook of categorical algebra 1. Basic category theory. Encyclopedia of Mathematics and its applications, Cambridge University Press, 1994, 360 pp.

[Bo2] F. Borceux. Handbook of categorical algebra 2. Categories and structures. Encyclopedia of Mathematics and its applications, Cambridge University Press, 1994, 360 pp. 
[CaGa] J.G. Cabello, A.R. Garzn. Closed model structures for algebraic models of n-types. Journal of Pure and Applied Algebra 103 (3) (1995), 287-302.

[Cra] S.E. Crans. Quillen closed model structures for sheaves. Journal of Pure and Applied Algebra 101, 1995, pp. 35-57.

[DeMi] P. Deligne, J. Milne. Tannakian categories. In: Hodge cycles, motives and Shimura varieties, Springer LN in Math., 900 (1982), 101-228.

[Fr] B. Fresse. Koszul duality for operads and homology of partition posets.

[Ga] W. L. Gan. Koszul duality for dioperads. Math. Res. Lett. 10:1 (2003), $109-124$.

[GeKa1] E. Getzler, M. Kapranov. Cyclic operads and cyclic homology. In: Geometry, Topology and Physics for Raoul Bott (ed. by S.-T. Yau), International Press 1995, 167-201.

[GeKa2] E. Getzler, M. Kapranov. Modular operads. Compositio Math., 110:1 (1998), 65-126.

[GiKa] V. Ginzburg, M. Kapranov. Koszul duality for operads. Duke Math. J., 76:1 (1994), 203-272.

[GoMa] A. Goncharov, Yu. Manin. Multiple zeta-motives and moduli spaces $\bar{M}_{0, n}$. Compos. Math. 140:1 (2004), 1-14. Preprint math.AG/0204102

[GrM] S. Grillo, H. Montani. Twisted internal COHOM objects in the category of quantum spaces. Preprint math.QA/0112233

[Hin] V. Hinich. Homological algebra of homotopy algebras. Communications in Algebra 25(10), 1997, pp. 3291-3323.

[KaMa] M. Kapranov, Yu. Manin. Modules and Morita theorem for operads. Am. J. of Math., 123:5 (2001), 811-838. Preprint math.QA/9906063

[KoMa] M. Kontsevich, Yu. Manin. Gromov-Witten classes, quantum cohomology, and enumerative geometry. Comm. Math. Phys., 164:3 (1994), 525-562.

[KS] G.M. Kelly, R. Street. Review of the elements of 2-categories in Category Seminar Lecture Notes in Mathematics 420, Springer 1974, 75-103.

[LoMa] A. Losev, Yu. Manin. Extended modular operad. In: Frobenius Manifolds, ed. by C. Hertling and M. Marcolli, Vieweg \& Sohn Verlag, Wiesbaden, 2004, 181-211. Preprint math.AG/0301003

[Ma1] Yu. Manin. Some remarks on Koszul algebras and quantum groups. Ann. Inst. Fourier, Tome XXXVII, f. 4 (1987), 191-205.

[Ma2] Yu. Manin. Quantum groups and non-commutative geometry. Publ. de CRM, Université de Montréal, 1988, 91 pp.

[Ma3] Yu. Manin. Topics in noncommutative geometry. Princeton University Press, 1991, 163 pp. 
[Ma4] Yu. Manin. Notes on quantum groups and quantum de Rham complexes. Teoreticheskaya i Matematicheskaya Fizika 92:3 (1992), 425-450. Reprinted in Selected papers of Yu. I. Manin, World Scientific, Singapore 1996, 529-554.

[Mar] M. Markl. Operads and PROPs. Preprint math.AT/0601129.

[MarShSt] M. Markl, St. Shnider, J. Stasheff. Operads in Algebra, Topology and Physics. Math. Surveys and Monographs, vol. 96, AMS 2002.

[MarkSh] I. Markov, Y. Shi. Simulating quantum computation by contracting tensor network. Preprint quant-ph/0511069

[Mer] S. Merkulov. PROP profile of deformation quantization and graph complexes with loops and wheels. Preprint math.QA/0412257

[PP] A. Polishchuk, L. Positselski. Quadratic algebras. University Lecture series, No. 37, AMS 2005.

[Pow] A. J. Power. A 2-categorical pasting theorem. Journ. of Algebra, 129 (1990), 439-445.

[Q] D. Quillen Homotopical Algebra. Lecture Notes in Mathematics, Vol. 43, Springer, Berlin, 1967.

[R] C. Rezk. Spaces of algebra structures and cohomology of operads. Ph.D. Thesis, Massachusetts Institute of Technology, Cambridge, MA, 1996.

[S] J. Spaliński. Strong homotopy theory of cyclic sets. Journal of Pure and Applied Algebra 99 (1) (1995) 35-52.

[Va1] B. Vallette. A Koszul duality for PROPs. Preprint math.AT/0411542 (to appear in the Transactions of the AMS).

[Va2] B. Vallette. Manin's products, Koszul duality, Loday algebras and Deligne conjecture. Preprint math.QA/0609002

[Va3] B. Vallette. Free monoid in monoidal abelian categories. Preprint math.CT/0411543

[Zo] P. Zograf. Tensor networks and the enumeration of regular subgraphs. Preprint math.CO/0605256 